\title{
Creating Better Binary Classification Trees Using Non-Greedy Methods
}

\author{
Julian R. Andean
}

A thesis submitted to the Faculty of Graduate Studies and Research in partial fulfillment of the requirements for the degree of Master of Applied Science in Electrical Engineering

Ottawa-Carleton Institute of Electrical and Computer Engineering (OCIECE)

Department of Systems and Computer Engineering

Carleton University

Ottawa, Ontario, Canada, K1S 5B6

August 14, 2009

(C) Copyright 2009, Julian Andean 


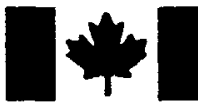

Library and Archives

Canada

Published Heritage

Branch

395 Wellington Street

Ottawa ON K1A ON4

Canada
Bibliothèque et

Archives Canada

Direction du

Patrimoine de l'édition

395 , rue Wellington

Ottawa ON K1A ON4

Canada
Your file Votre référence

ISBN: 978-0-494-60289-8

Our file Notre référence

ISBN: 978-0-494-60289-8
NOTICE:

The author has granted a nonexclusive license allowing Library and Archives Canada to reproduce, publish, archive, preserve, conserve, communicate to the public by telecommunication or on the Internet, loan, distribute and sell theses worldwide, for commercial or noncommercial purposes, in microform, paper, electronic and/or any other formats.

The author retains copyright ownership and moral rights in this thesis. Neither the thesis nor substantial extracts from it may be printed or otherwise reproduced without the author's permission.
AVIS:

L'auteur a accordé une licence non exclusive permettant à la Bibliothèque et Archives Canada de reproduire, publier, archiver, sauvegarder, conserver, transmettre au public par télécommunication ou par l'Internet, prêter, distribuer et vendre des thèses partout dans le monde, à des fins commerciales ou autres, sur support microforme, papier, électronique et/ou autres formats.

L'auteur conserve la propriété du droit d'auteur et des droits moraux qui protège cette thèse. $\mathrm{Ni}$ la thèse ni des extraits substantiels de celle-ci ne doivent être imprimés ou autrement reproduits sans son autorisation.
In compliance with the Canadian Privacy Act some supporting forms may have been removed from this thesis.

While these forms may be included in the document page count, their removal does not represent any loss of content from the thesis.
Conformément à la loi canadienne sur la protection de la vie privée, quelques formulaires secondaires ont été enlevés de cette thèse.

Bien que ces formulaires aient inclus dans la pagination, il n'y aura aucun contenu manquant. 


\begin{abstract}
Decision tree creation algorithms currently employ greedy methods to decide where to place separating surfaces, known as hyperplanes. A greedy hyperplane placement method is one which attempts to choose a hyperplane whose placement results in the best immediate improvement of the performance of the tree. This thesis proposes a set of non-greedy hyperplane placement methods and a set of policies that determine which hyperplane placement method to use when. These non-greedy hyperplane placement methods attempt to place hyperplanes which may not produce the best immediate improvement to a tree's performance, but whose inclusion may produce a better overall tree once the entire tree has been constructed. This thesis documents the performance of numerous policies and compares their performance against the current state of the art methods. The results demonstrate that policies that make use of nongreedy hyperplane placement methods are generally able to construct better decision trees than their greedy counterparts.
\end{abstract}




\section{Acknowledgements}

I would like to thank Dr. John Chinneck for the continuous support and guidance during the development of this thesis paper. I would also like to thank my parents, better half and friends for their support and encouragement. 


\section{TABLE OF CONTENTS}

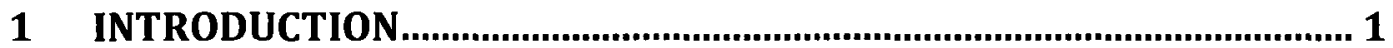

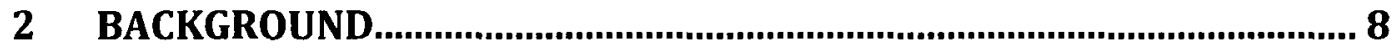

2.1 Binary Classification Trees .............................................................................8

2.1.1 Training a Binary Classification Tree ..............................................................9

2.1.2 Metrics for Hyperplane Placement ....................................................... 10

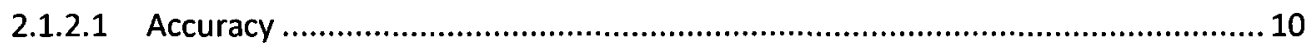

2.1.2.2 Gini Index............................................................................... 10

2.1.2.3 Entropy...................................................................................... 13

2.1.2.4 Receiver Operating Characteristic (ROC) .......................................... 14

2.1.2.5 Cost Function ................................................................................ 18

2.1.3 Hyperplane Creation Methods ........................................................... 18

2.1.3.1 Univariate Hyperplane Creation Methods.................................................. 18

2.1.3.2 Multivariate Hyperplane Placement Methods............................................ 22

2.1.4 Avoidance of Over-Fitting................................................................... 27

2.1.4.1 Stopping Criterion ............................................................................ 28

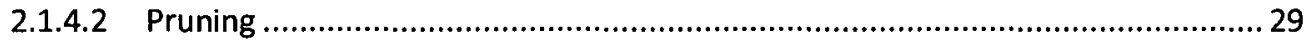

2.1.4.3 A Comparison of Pruning to Stopping Criteria ............................................ 31

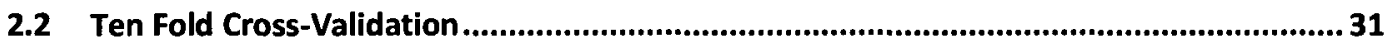

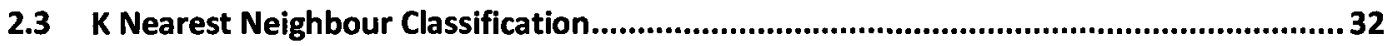

3 A REVIEW OF THE STATE OF THE ART ........................................ 33

3.1 Homogenous Hyperplane Placement .............................................................. 33

3.1.1 Univariate Hyperplane Placement ....................................................... 33

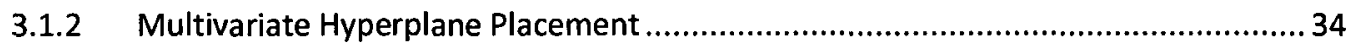

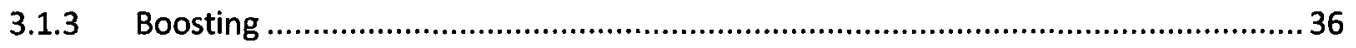

3.2 Heterogeneous Hyperplane Placement ...............................................................36

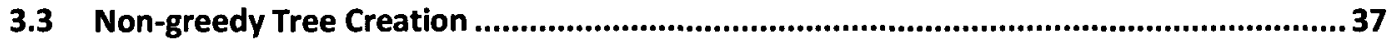

4 CREATING BETTER BINARY CLASSIFICATION TREES..........................39

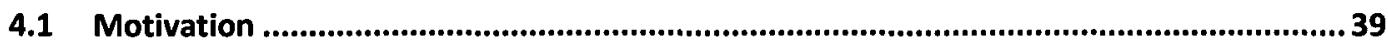

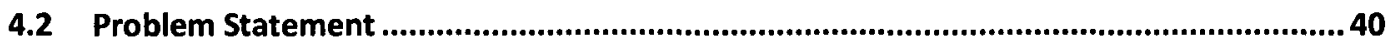

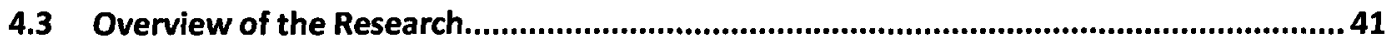

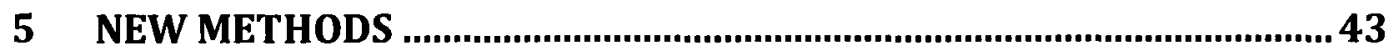

5.1 New Non-Greedy Hyperplane Placement Methods ...............................................43

5.1.1 Mixed Zone Cut.................................................................................. 43 
5.1.2 Edge Following (EF) Hyperplane Placement Methods .........................................46

5.1.2.1 Edge Following (EF) Class Isolation Pure Cut Hyperplane Placement Method ..... 50

5.1.2.2 Edge Following (EF) Class Isolation Greedy Cut Hyperplane Placement Method . 53

5.1.2.3 Edge Following (EF) Mixed Isolation Pure Cut Hyperplane Placement Method ... 62

5.1.2.4 Edge Following (EF) Mixed Isolation Greedy Cut Hyperplane Placement Method 63

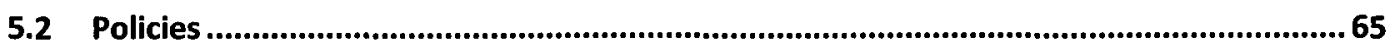

5.2.1 Policies Employing Greedy Hyperplane Placement Techniques .................................65 65

5.2.2 Policies Employing Non-Greedy Hyperplane Placement Techniques..........................65 65

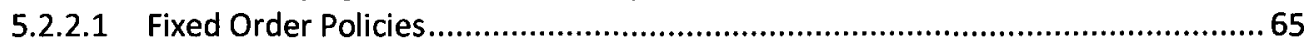

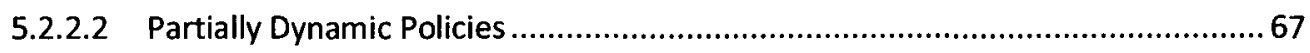

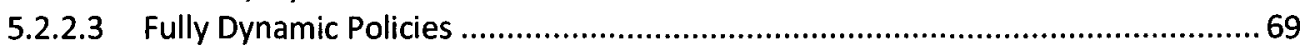

5.2.2.4 Tree Growth Termination Within Policies ................................................... 70

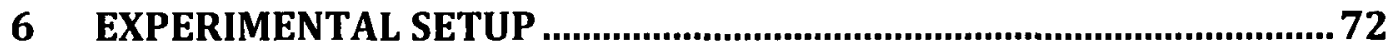

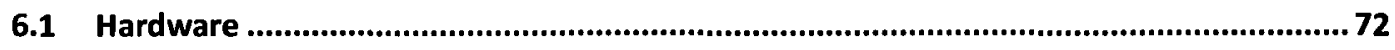

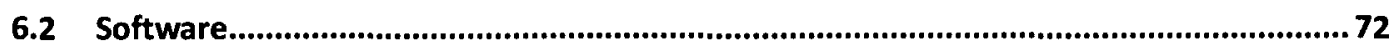

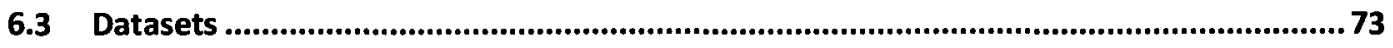

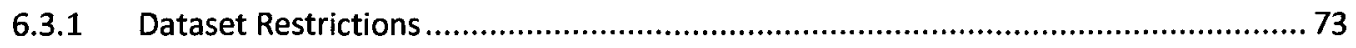

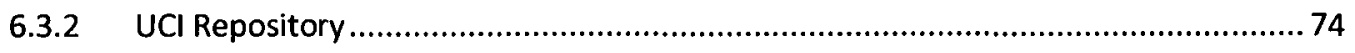

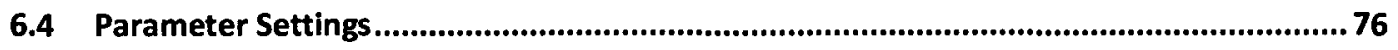

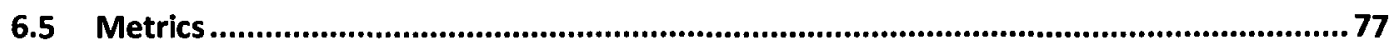

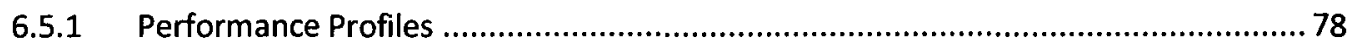

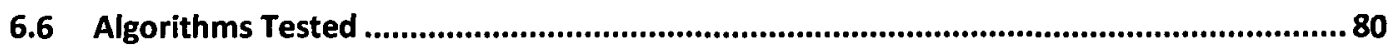

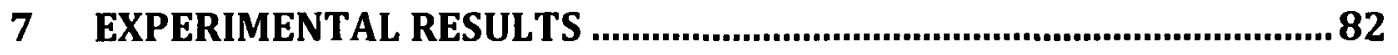

7.1 A Comparison of Policies Vs. the Greedy Hyperplane Placement Method ......................... 88

7.2 A Comparison of Policies Vs. Moghrabi's (2003) Results..............................................91

7.3 A Comparison of Policies Vs. Commercial Classifiers .............................................................94

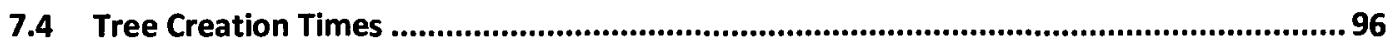

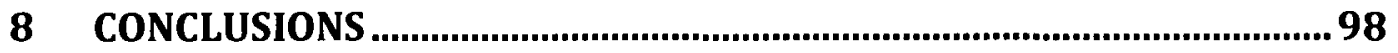

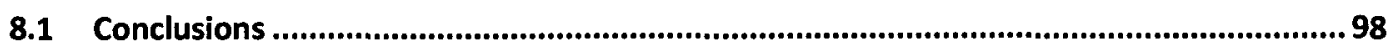

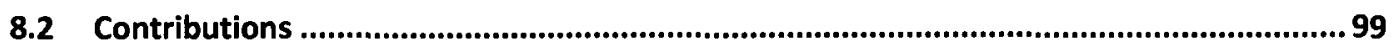

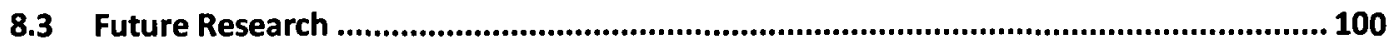

9 APPENDIX A - ARTIFICIAL DATASETS .................................................. 101 


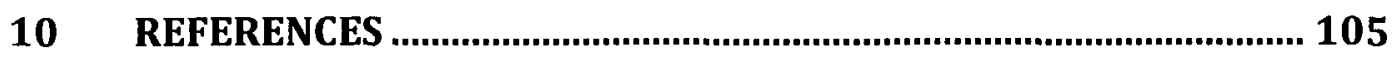




\section{List of Tables}

TABLE 1: A CONFUSION MATRIX

TABLE 2: THE PERFORMANCE OF 3 DIFFERENT CLASSIFIERS ON ONE DATASET 17

TABLE 3: THE AND DATASET

TABLE 4: FIXED ORDER POLICIES

TABLE 5: PARTIALLY DYNAMIC POLICIES

66

TABLE 6: UCI DATASETS USED IN THIS THESIS

68

TABLE 7: AVERAGE PERFORMANCE OF 3 GREEDY HYPERPLANE PLACEMENT METHODS.

TABLE 9: AVERAGE ACCURACY AND STANDARD DEVIATION OVER ALL DATASETS 


\section{List of Figures}

FIGURE 1: A CLASSIFICATION TREE PREDICTING HEART ATTACK SURVIVAL (BREIMAN ET AL., 1984) 2

FIGURE 2: THE SAME DATASET, SPLIT ON THE LEFT BY A GREEDY HYPERPLANE PLACEMENT METHOD, AND

SPLIT ON THE RIGHT BY A NON-GREEDY HYPERPLANE PLACEMENT METHOD.

FIGURE 3: THE FINAL TREES, THE ONE ON THE LEFT CREATED USING A GREEDY HYPERPLANE PLACEMENT METHOD, AND THE ONE ON THE RIGHT CREATED USING A NON-GREEDY HYPERPLANE PLACEMENT METHOD.

FIGURE 4: AN EXAMPLE INCOME DISTRIBUTION GRAPH (GOWAN, 2008)

FIGURE 5: A PLOT OF ENTROPY VS. CLASS P'S PROBABILITY (DMS, 2001)

FIGURE 6: THE PERFORMANCE OF 3 CLASSIFIERS IN AN ROC GRAPH

FIGURE 7: A SAMPLE DATA SET

FIGURE 8: A FIRST UNIVARIATE SPLIT

FIGURE 10: A UNIVARIATE BINARY CLASSIFICATION TREE

FIGURE 9: THE 2 UNIVARIATE SPLITS CORRECTLY CLASSIFY ALL THE DATA

FIGURE 11: A SINGLE MULTIVARIATE HYPERPLANE CLASSIFIES ALL THE DATA CORRECTLY

FIGURE 12: AN EXAMPLE IN WHICH IT WOULD TAKE HUNDREDS OF UNIVARIATE HYPERPLANES IN A STAIRCASE PATTERN TO CLASSIFY THIS DATASET.

FIGURE 13: THE SOLUTION FOR THE AND DATASET

FIGURE 14: TEST SET VS. TRAINING SET PERFORMANCE

FIGURE 15: THE FIRST HYPERPLANE PLACED USING 3 DIFFERENT GREEDY ALGORITHMS: ACCURACY, GINI, AND ENTROPY

FIGURE 16: THE FINAL TREE CREATED USING A GREEDY HYPERPLANE PLACEMENT METHOD WHICH MAXIMIZES FOR ACCURACY.

FIGURE 17: THE MIXEDBULLSEYE DATASET

FIGURE 18: THE TRANSFORMED DATASET. "+" SYMBOLS REPRESENT CLASS P DATA POINTS, AND CIRCLES REPRESENTS CLASS M DATA POINTS

FIGURE 19: A PURE CUT TAKEN ON THE TRANSFORMED DATASET

FIGURE 20: THE SAME HYPERPLANE SEEN ON THE ORIGINAL DATASET

FIGURE 21: AFTER 4 MIXED ZONE CUTS, THE HOMOGENOUS GROUP OF CLASS 1 DATA POINTS HAS BEEN ISOLATED.

FIGURE 22: THE FIRST HYPERPLANE PLACED BY A PURE CUT HYPERPLANE PLACEMENT METHOD

FIGURE 23: THE FINAL TREE CREATED BY A PURE CUT HYPERPLANE PLACEMENT METHOD

FIGURE 24: THE FIRST HYPERPLANE, PLACED USING A EDGE FOLLOWING HYPERPLANE PLACEMENT METHOD

FIGURE 25: THE FINAL TREE, CREATED USING AN EF HYPERPLANE PLACEMENT METHOD

FIGURE 26: THE KNN SUBSET OF THE HALFHOURGLASS DATASET FOR $K=20$

FIGURE 27: A PURE CUT PLACED IN THE KNN SUBSET.

FIGURE 28: THE SAME PURE CUT FROM THE KNN SUBSET, PLACED ON THE ORIGINAL DATASET.

FIGURE 29: THE FINAL TREE, CREATED BY THE PLACEMENT OF TWO EF CLASS ISOLATION PURE CUT HYPERPLANES.

FIGURE 30: A GREEDY CUT PLACED IN THE KNN SUBSET DOESN'T WORK

FIGURE 31: THE LINEAR SUBSET

FIGURE 32: A GREEDY CUT PLACED IN THE LINEAR SUBSET

FIGURE 33: AN EF CLASS ISOLATION GREEDY CUT ON THE ORIGINAL DATASET.

FIGURE 34: A SAMPLE DATASET WITH A LARGE MIXED ZONE.

FIGURE 35: A PURE CUT IS TAKEN ON THE KNN SUB-DATASET. THE SUB-DATASET IS THEN FURTHER REDUCED TO THE LINEAR SUBSET.

FIGURE 36: A GREEDY CUT IS PLACED IN THE LINEAR SUBSET, BUT THIS GREEDY CUT WHEN VIEWED AGAINST THE FULL DATA SET DOESN'T MAKE MUCH SENSE.

FIGURE 37: THE INITIAL ITERATION, AT THIS STAGE, THE MIXED ZONE CONTAINED IN THE LINEAR SUBSET EXTENDS ONLY TO ABOUT $X=-20$ 
FIGURE 38: AFTER 2 MORE ITERATIONS, A LARGER SECTION OF MIXED ZONE POINTS IS NOW INCORPORATED INTO THE LINEAR SUBSET.

FIGURE 39: AFTER A COUPLE MORE ITERATIONS, THE COMPLETE MIXED ZONE IS NOW INCORPORATED INTO THE LINEAR SUBSET.

FIGURE 40: THE FINAL HYPERPLANE, SEEN AGAINST THE LINEAR SUBSET AND AGAINST THE ORIGINAL DATASET

FIGURE 41: THE PERFORMANCE PROFILE FOR THE BEST 7 FIXED ORDER POLICIES.

FIGURE 42: THE PERFORMANCE PROFILE FOR THE 4 PARTIALLY DYNAMIC POLICIES.

FIGURE 43: THE PERFORMANCE PROFILE FOR THE FULLY DYNAMIC POLICIES.

FIGURE 44: A PERFORMANCE PROFILE PLOTTING THE PERFORMANCE OF POLICIES 3 AND 4 AGAINST THE GREEDY METHOD

FIGURE 45: A PERFORMANCE PROFILE PLOTTING THE PERFORMANCE OF POLICIES 10 AND 13 AGAINST THE GREEDY METHOD.

FIGURE 46: A PERFORMANCE PROFILE PLOTTING THE PERFORMANCE OF POLICIES 16 AND 18 AGAINST THE GREEDY METHOD.

FIGURE 47: A PERFORMANCE PROFILE PLOTTING THE PERFORMANCE OF POLICIES 20 AND 21 AGAINST

THE GREEDY METHOD.
FIGURE 48: A PERFORMANCE PROFILE COMPARING THE RESULTS FROM MOGHRABI'S (2003) THESIS AGAINST VARIOUS COMBINATIONS OF NON-GREEDY POLICIES.

FIGURE 49: A PERFORMANCE PROFILE COMPARING THE STATE OF THE ART TREE CREATION METHODS AGAINST THE BEST PERFORMING NON-GREEDY POLICY

FIGURE 50: THE BEST OF ALL POLICIES AGAISNT FREELY AVAILABLE COMMERCIAL CLASSIFIERS.

FIGURE 51: THE BEST OF ALL POLICIES AGAINST FREELY AVAILABLE COMMERCIAL CLASSIFIERS.

FIGURE 52: BULLSEYE AND BULLSEYEM DATASETS

FIGURE 53: DIAGONALSPLIT AND DIAGONALSPLITM DATASETS 


\section{List of Algorithms}

ALGORITHM 1: CHINNECK'S (2009) MOST RECENT LP BASED HYPERPLANE PLACEMENT ALGORITHM 35

ALGORITHM 2: MIXED ZONE HYPERPLANE PLACEMENT

ALGORITHM 3: EF CLASS ISOLATION PURE CUT HYPERPLANE PLACEMENT

ALGORITHM 4: EF CLASS ISOLATION GREEDY CUT HYPERPLANE PLACEMENT

ALGORITHM 5: EF MIXED ISOLATION PURE CUT HYPERPLANE PLACEMENT

ALGORITHM 6: EF MIXED ISOLATION GREEDY CUT HYPERPLANE PLACEMENT

ALGORITHM 7: PARTIALLY DYNAMIC POLICIES 


\section{List of Acronyms}

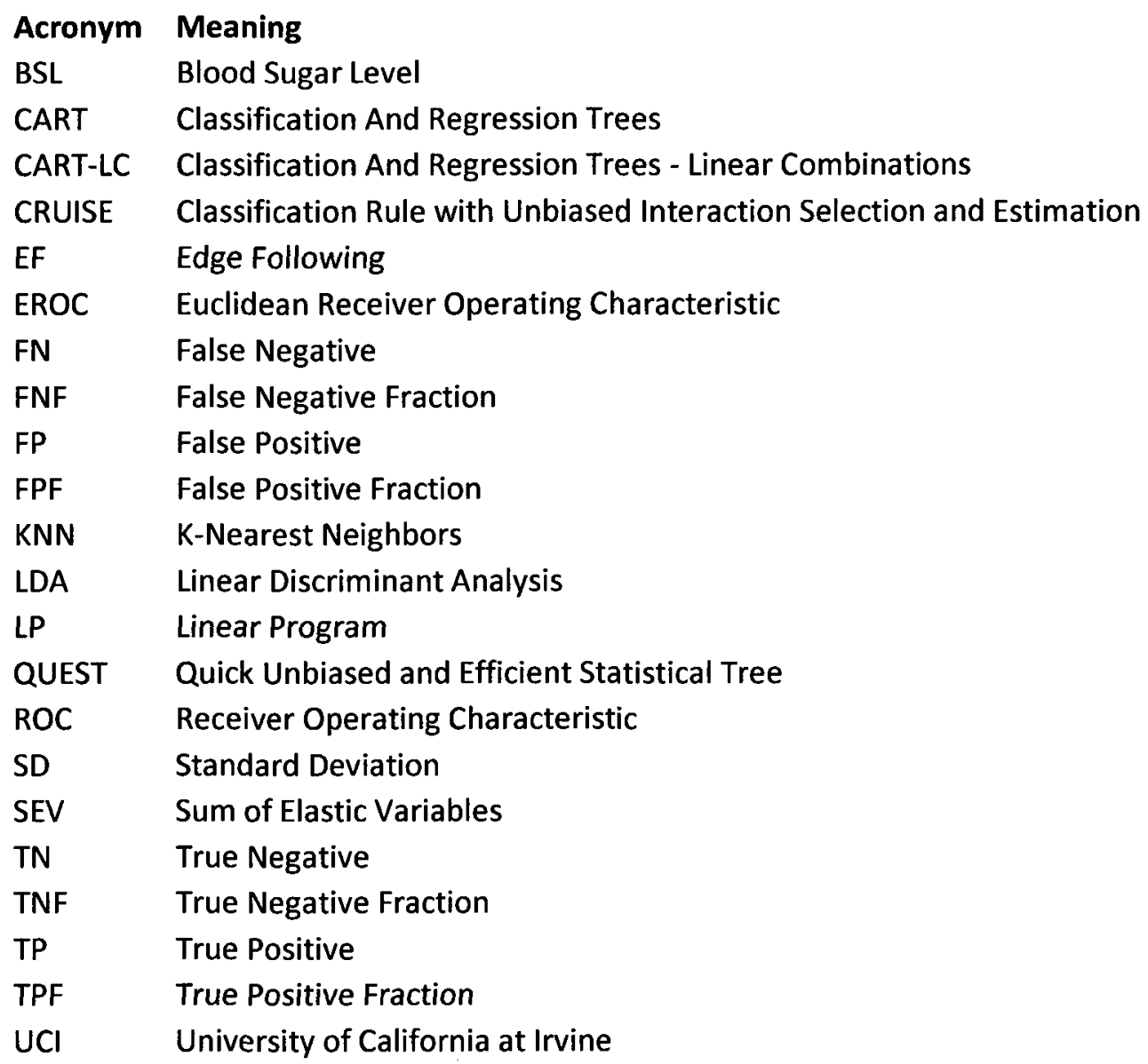




\section{Introduction}

Classification, in the field of machine intelligence, is a process in which the value of an attribute of a given object is predicted based on the known values of other attributes of that same object. The attribute whose value is being predicted is referred to as the class, and the attributes whose values are used to help make that prediction are called predictors. Consider a biologist who is measuring the length and width of the sepals and petals of iris plants, and using those values to predict what kind of iris plant those measurements were taken from. The length and width of the sepals and petals would be the predictors, and the type of iris plant would be the class.

A decision tree is a popular classification method that represents a set of tests and their results using a graph. A node in the graph represents a test to be performed on an object's predictors. Arcs represent the possible outcomes of that test. Thus an object traverses the graph by starting at the first node, and progressing down the graph, choosing subsequent nodes to move to based upon the result of the test performed at the current node. Once an object has reached the end of the graph, which would be a node with no arcs leading from it, a class is assigned to that object. Figure 1 depicts a decision tree that predicts the likelihood that a heart attack patient will survive for 30 days: 


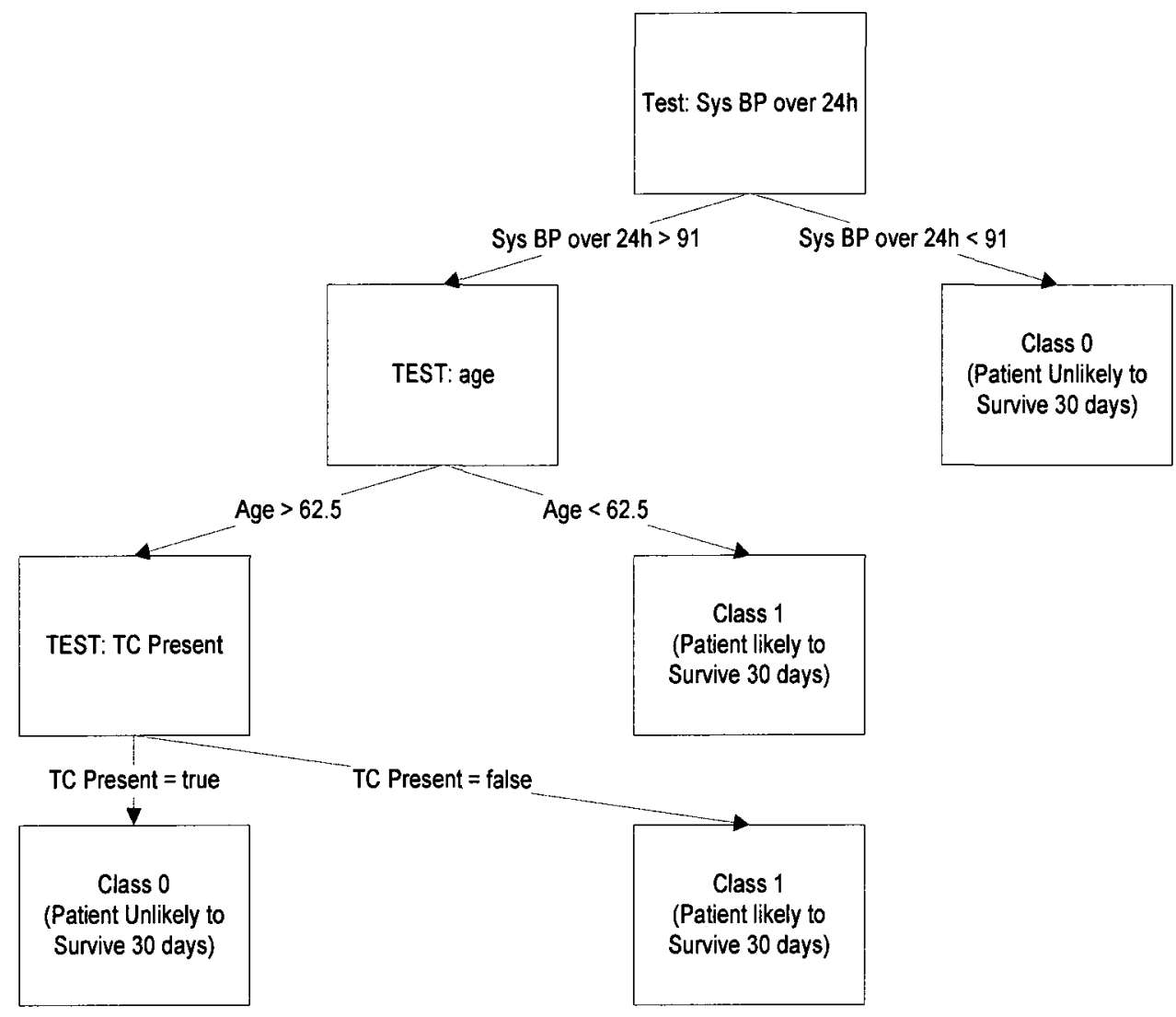

Figure 1: A classification tree predicting heart attack survival (Breiman et al., 1984)

There are two basic types of decision trees; Regression Trees and Classification Trees. Regression trees are used when the variable being classified is continuous. For example a tree designed to predict the probability of severe weather would be a regression tree. After traversing the tree, a number representing the probability of encountering severe weather would be produced. Classification trees are used when the variable being classified has discrete values. A tree that examines a vehicle's properties and then predicts whether that vehicle is a "car" or "truck" or "bike" would be an example of a classification tree. A binary classification tree is one in which the class being predicted has only two possible values, such as true or false, positive or negative, etc... Figure 1 is an example of a binary classification tree. 
Decision tree learning is the process by which a decision tree is created. This process requires a set of data points, called the training dataset, whose classes are known. The decision tree learning algorithm then recursively divides the training dataset into subdatasets. When linear separating surfaces are used, the division that subdivides a dataset is referred to as a hyperplane, and the method used to subdivide the data is referred to as the hyperplane placement method.

Hyperplane placement methods fall into two groups: greedy and non-greedy methods. For any given data subset, a greedy hyperplane placement method will attempt to create a hyperplane which will achieve the best result, for a given metric, for that specific data subset. This emphasis on local maximization of a given metric can result in a tree whose overall performance for that same metric is poor. A non-greedy hyperplane placement method attempts to partition a data subset in such a way as to maximize the final overall tree's performance for a given metric. Figure 2 shows a training dataset, in which " $x$ " represents class 1 data points and squares represent class 0 data points, that has been subdivided once with a greedy hyperplane placement method, and once with a nongreedy hyperplane placement method. 


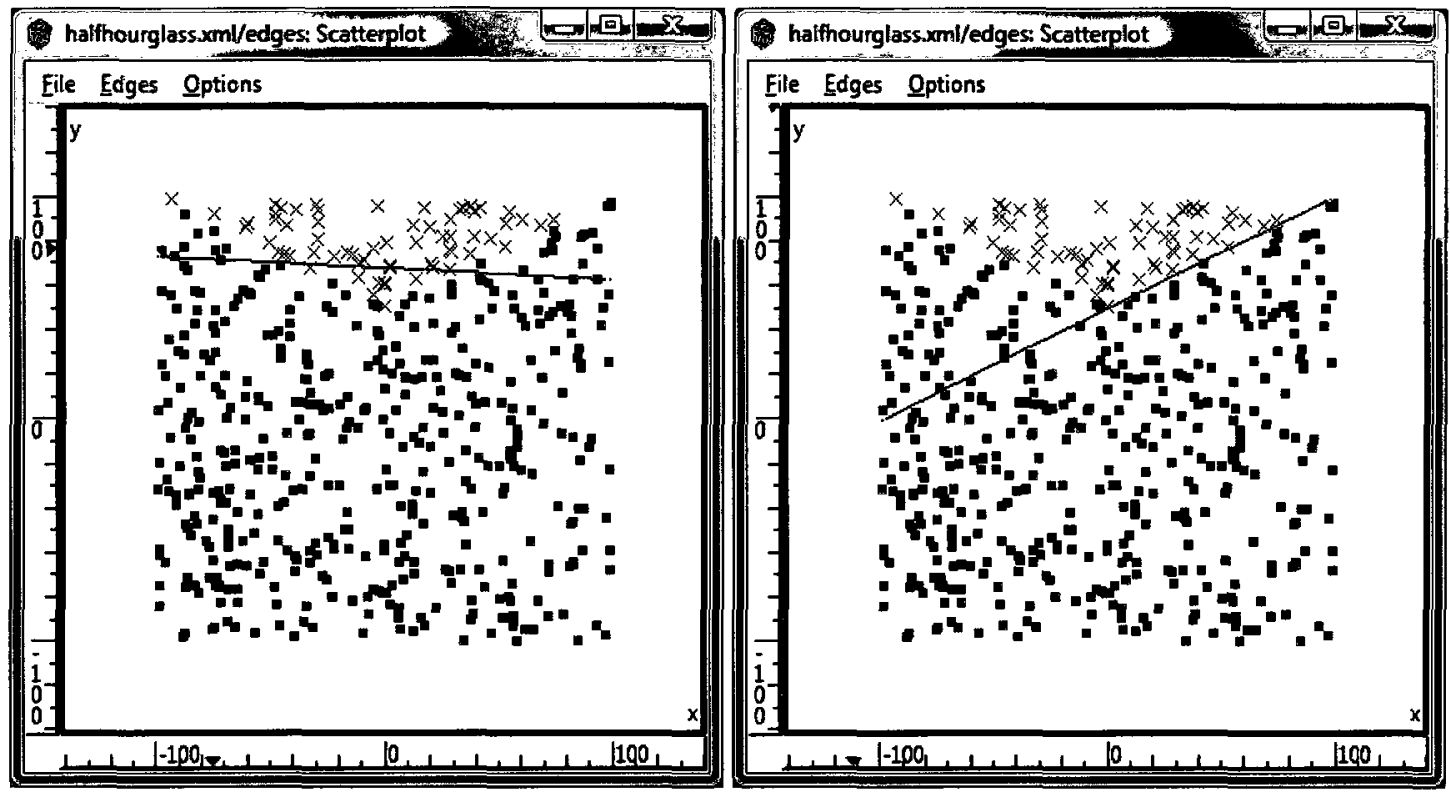

Figure 2: The same dataset, split on the left by a greedy hyperplane placement method, and split on the right by a non-greedy hyperplane placement method.

Using accuracy as the measure of how well a tree performs, a comparison of the two graphs shows that the greedy method has provided a more accurate subdivision of the data points. Note the small triangle of class 1 data points that the greedy method has misclassified at this stage of tree creation. The misclassification of these points has allowed more class 0 data points to be classified correctly, and is a good illustration of local maximization. Once both trees are fully branched, the resulting graphs are shown in Figure 3. 

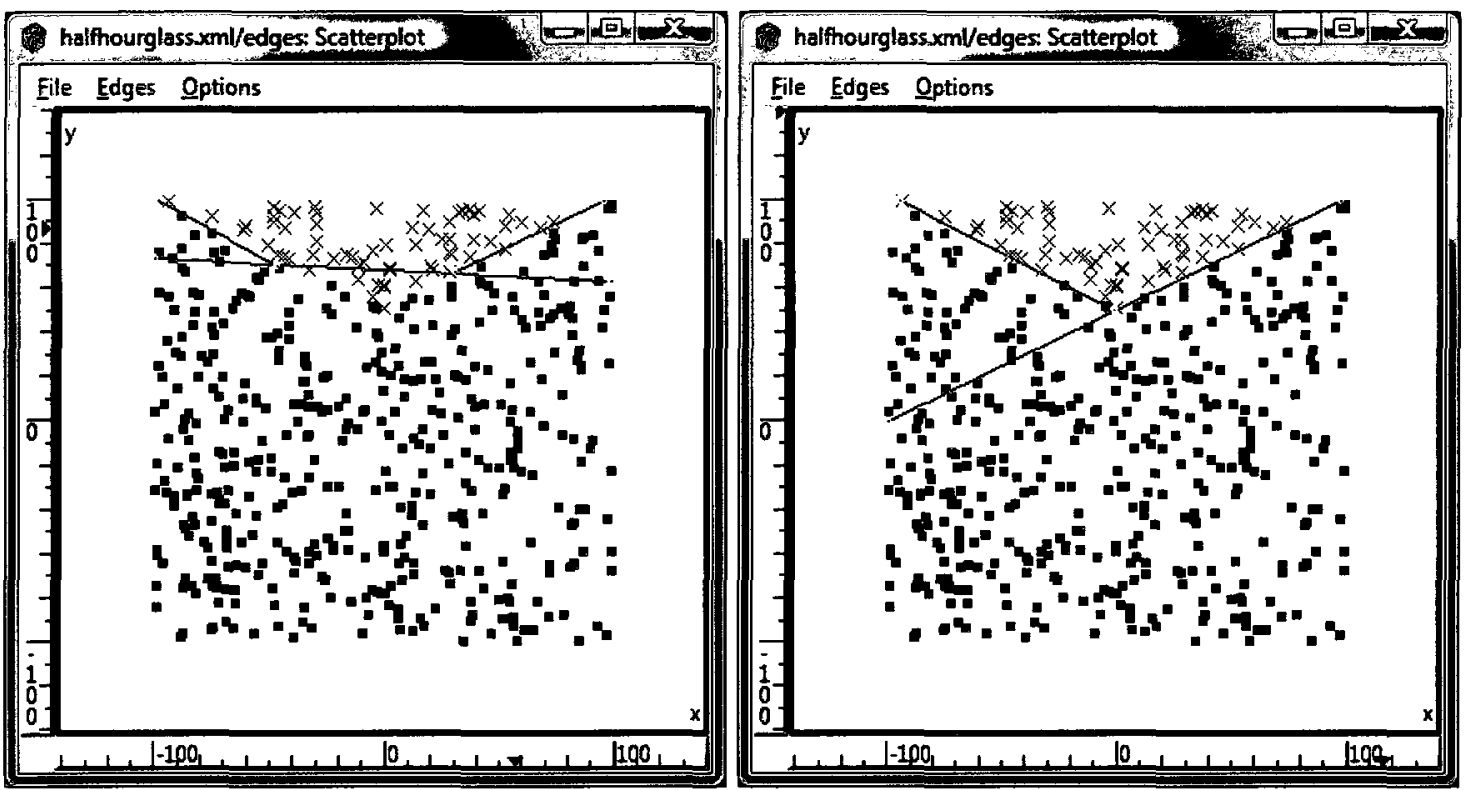

Figure 3: The final trees, the one on the left created using a greedy hyperplane placement method, and the one on the right created using a non-greedy hyperplane placement method.

The graph on the left shows that the final tree created using the greedy hyperplane placement method has several misclassified data points. The greedy algorithm was unable to find a way to reclaim the triangle of class 1 data points that were misclassified by the first hyperplane. The graph on the right shows that the tree created using a nongreedy hyperplane placement method has no misclassified data points. The greedy method maximized for the accuracy of the first hyperplane, but in the end, produced an inferior overall tree. The non-greedy method produced a first branch which was less accurate than its greedy counterpart, but the selection of this initial branch allowed for the creation of a better overall tree in the end.

Research in the field of decision tree learning has focused for the most part on developing greedy hyperplane placement methods. Non-greedy hyperplane placement methods have received less attention.

This thesis focuses on using non-greedy hyperplane placement methods to create better binary classification trees. Five new non-greedy hyperplane placement methods 
are introduced in Section 5.1. These methods are an extension of Chinneck's (2009) basic greedy hyperplane placement algorithm, described in Section 3.1. These 5 methods can be split up into 2 basic categories.

The first category is referred to as Zone Cuts. Section 3.1 details a pure zone cut created by Chinneck (2009). A pure zone cut is a hyperplane placement method which attempts to isolate the largest homogeneous group of data points that can be found. Section 5.1.1 introduces a new zone cut in which a mixed zone cut is taken. A mixed zone cut is a hyperplane placement method which attempts to isolate the largest heterogeneous group of data points that can be found. A homogenous group of data points is one in which all data points belong to the same class. A heterogeneous group of data points is one in which more than one class of data point is present in the group.

The second category is referred to as Edge Following (EF) Cuts. These 4 different subdivision methods, detailed in Sections 5.1.2.1- 5.1.2.4, attempt to create a hyperplane along the longest linear or near-linear region of intersection between two different groups of data points. "Different groups" can refer to two homogenous groups of different class type, or a heterogeneous group and a homogenous one.

A number of policies are proposed in Section5.2.2. These determine what hyperplane placement method to apply at a given node. There are a significant number of policies, but they can be broken down into three basic categories.

- Fixed Order Policies apply hyperplane placement methods in a specific order. Policies 1 to 15 are fixed order policies, and are discussed in section 5.2.2.1. 
- Partially Dynamic Policies use a merit based selection process that chooses, from a limited range of options, which hyperplane placement method to use at a given node. Policies 16 to 19 are partially dynamic policies, and are discussed in section 5.2.2.2.

- Dynamic Policies offer the most flexibility in choosing which hyperplane placement method is applied at any given node. Policies 20 to 23 are dynamic policies, and are discussed in section 5.2.2.3.

A description of the experimental setup is given in Chapter 1, and the empirical results achieved are presented in Chapter 1 . These results show that non-greedy hyperplane placement methods allow the construction of better binary classification trees than their greedy counterparts. A comparison of the performance of the various policies shows that a parallel development of several trees using fixed order and fully dynamic policies performed best, while the best single tree creation policy was Fixed Order Policy 4. 


\section{Background}

\subsection{Binary Classification Trees}

A binary classification tree is represented as a graph in which each node, excluding terminal nodes, has exactly two sub-nodes. Each node in the graph represents a test that is performed using an object's predictors, and each arc in a tree represents a possible outcome of that test. A test is a comparison of a predictor, or combination of predictors, against a specific value. Some examples of tests are:

1) A biologist attempting to classify hares and rabbits may use ear length as a predictor to test against. Subjects with an ear length $>30 \mathrm{~cm}$ are assigned to the "hare" group, while those with an ear length $\leq 30 \mathrm{~cm}$ are assigned to the "rabbit" group.

2) A doctor testing for diabetes might choose a patient's blood sugar level (BSL) as a predictor to test against, with the actual test being BSL $>50$. Patients with a BSL $>50$ are assigned to the diabetic group, while those whose $\mathrm{BSL} \leq 50$ are assigned to the non-diabetic group.

The relationship between different nodes in a tree can be described as follows. Node $\alpha$ is said to be node $\beta$ 's parent if any of the possible results of node $\alpha$ 's test can result in a data point being assigned to node $\beta$. Node $\beta$ is said to be node $\alpha$ 's child if any of the possible results of node $\alpha$ 's test can result in a data point being assigned to node $\beta$. Node $\alpha$ is said to be node $\beta$ 's ancestor if it is possible to traverse from node $\beta$ to node $\alpha$ upwards in the tree. Node $\beta$ is said to be node $\alpha$ 's descendent if $\alpha$ is node $\beta$ 's ancestor. Node $\alpha$ is said to be node $\beta$ 's sibling if both nodes share the same parent. 
There are three types of nodes that can be found in a tree. A root node is the first node in the tree. A root node has no parent, and all other nodes in the tree are its descendents. A leaf node is a node which has no children. A leaf node will have a class associated with it, and data points that have traversed a tree and arrived at a leaf node are assigned the class that is associated with that leaf node. A branch node is a node that has both parent and child nodes associated with it. Data points are assigned to a branch node by its parent, and are then reassigned to a child node by the branch node.

\subsubsection{Training a Binary Classification Tree}

Given a training dataset, the process for creating a tree is as follows:

1) An initial node, the root node, is added to the tree. This node is comprised of the entire training dataset.

2) A list of unexamined nodes is created. This list will initially contain only the root node.

3) While the list of unexamined nodes is not empty:

a. Choose a node to examine.

b. If the data in the node can be subdivided then:

i. Choose a test to subdivide the data in that node.

ii. For each possible outcome of the selected test, create a child node and add it to the tree and the list of unexamined nodes.

iii. Partition the parent node's data points amongst the child nodes using the selected test. 
iv. Remove the parent node from the list of nodes to be examined. If the parent node is not the root, then the parent node is now labeled as a branch node.

c. If the data cannot be subdivided then:

i. The node is removed from the list of nodes to be examined.

ii. The node is now labeled as a leaf node.

\subsubsection{Metrics for Hyperplane Placement}

A metric is a measurement that is used to rate the performance of a hyperplane. During the tree creation process, there will generally be several possible hyperplanes that can be used to subdivide the data points of a given node. The hyperplane chosen will usually be the one that performs best for a given metric.

\subsubsection{Accuracy}

For any given candidate hyperplane, the overall accuracy of the hyperplane is the ratio of the number of correctly classified data points to the total number of data points. The higher the ratio achieved, the higher the accuracy of the candidate hyperplane.

\subsubsection{Gini Index}

The Gini Index (Gini, 1921) was originally devised as a way to measure income inequality in a population. If everyone in a given population has the same income, then a plot of the cumulative percentage of income against the cumulative percentage of the population produces a straight line from $(0,0)$ to $(100,100)$. This line is known as the Perfect Distribution Line. In reality, the lower income segment of the population earns a lower percentage of the total income, and the higher income segment of the population 


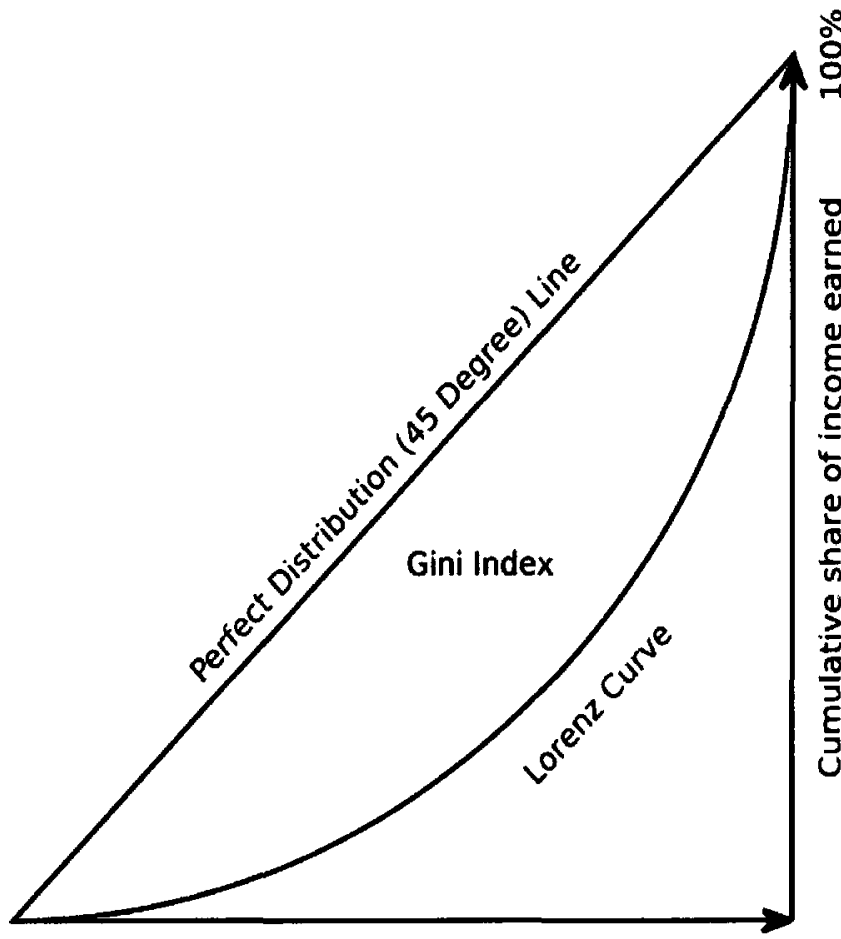

Cumulative share of people from lower income $100 \%$ earns a higher percentage of the total income. This reality, when plotted, results in a curve known as the Lorenz Curve. The Gini Index is defined as the ratio of the area between the Lorenz curve and the Perfect Distribution Line, and the total area under the Perfect Distribution Line. A Gini

Figure 4: An example income distribution graph (Gowan, 2008)

Index of 0 means that a population's wealth distribution follows the Perfect Distribution Line, and a Gini Index of 1 indicates that all wealth is held by 1 person.

The Gini Index was modified for the CART algorithm (Breiman et al., 1984) as a means of measuring the purity of a dataset. For any given dataset, the modified Gini Index for dataset $S$ is:

$$
G(S)=1-\sum_{j}[p(j)]^{2}
$$

Where $\mathrm{p}(\mathrm{j})$ represents the probability that a data point belonging to class $j$ is found in the dataset. If we have $n$ total data points, and $m$ points belong to class $j$, then:

$$
p(j)=\frac{m}{n}
$$


If all the data points in a dataset belong to class $j$, meaning that the dataset is pure, then $m=n$, and the Gini index is:

$$
G(S)=1-\left(\frac{n}{n}\right)^{2}=0
$$

If a dataset has $n$ total data points, with an equal number of data points assigned to each of $k$ classes, then the modified Gini Index is:

$$
\begin{aligned}
G(S)= & 1-\sum_{1}^{k}\left(\frac{n}{n * k}\right)^{2} \\
& =1-\frac{k}{k^{2}} \\
& =1-\frac{1}{k}
\end{aligned}
$$

Since the goal of classification is to partition a dataset into sub-datasets comprised of data points from a single class, a modified Gini Index of 0 is most desirable, and a Gini Index of $1-\frac{1}{k}$ (where $k$ represents the number of different classes) is least desirable.

The Gini Index assigned to a hyperplane is calculated by taking the weighted average of the Gini Indexes of the sub-datasets created by that hyperplane. Each sub-dataset's Gini Index is weighted according to the percentage of the parent's data points that are assigned to that sub-dataset by the hyperplane.

Consider an example in which a hyperplane partitions 20 class 1 data points and 10 class 0 data points. 16 class 1 data points and 4 class 0 data points are assigned to the class 1 node, while 6 class 0 data points and 4 class 1 data points are assigned to the class 0 node. The Gini index of the class 1 node would be: $1-\left(\frac{16}{20}\right)^{2}-\left(\frac{4}{20}\right)^{2}=0.32$, while the Gini index of the class 0 node would be: $1-\left(\frac{6}{10}\right)^{2}-\left(\frac{4}{10}\right)^{2}=0.48$. The Gini index 
for the hyperplane is the weighted average of the Gini Indices of the child nodes:

$\frac{20}{30} * 0.32+\frac{10}{30} * 0.48=0.373$.

\subsubsection{Entropy}

Ross Quinlan (1993) used Entropy as a means of measuring the suitability of a candidate hyperplane. Entropy is a measure of uncertainty, and is defined (Stinson, 2002) as being:

$$
H(X)=-\sum_{x \in X} \operatorname{Pr}[x] \log _{2} \operatorname{Pr}[\mathrm{x}]
$$

$X$ represents a finite set of discrete variables, and $x$ represents a member of that set. Applied to a data set, $X$ represents the data set, and $\operatorname{Pr}[\mathrm{x}]$ represents the probability that a data point of class $x$ is found in dataset $X$.

Consider a data set consisting of 10 data points, of class 0 or 1 . If all data points are of class 0 , then the entropy of this data set is:

$$
-1 * \log _{2}(1)-0 * \log _{2}(0)=0
$$

If the data points are split evenly amongst the two classes, the entropy of the data set is:

$$
-0.5 * \log _{2}(0.5)-0.5 * \log _{2}(0.5)=1
$$

If the distribution of the two classes varies from pure (e.g. 10 of one class and 0 of the other class), to evenly distributed (each class has 5 data points), Figure 5 shows a plot of the Entropy for a given class $p$ 's probability. An entropy of 0 represents a pure dataset, and the maximum entropy found represents a dataset whose data points are evenly distributed amongst the various classes found in that dataset. 


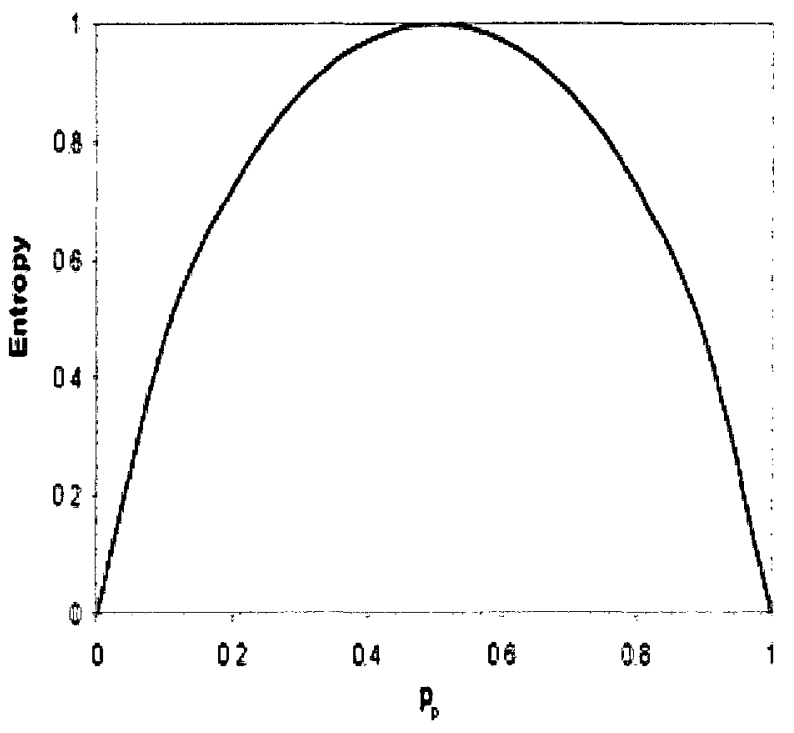

Figure 5: A plot of Entropy vs. Class p's Probability (DMS, 2001)
The entropy of a candidate hyperplane is found by taking the weighted sum of the entropies of the sub-datasets created by the candidate hyperplane. The weighting of a subdataset's entropy is proportional to the number of the parent node's data points

that are assigned to that sub-dataset.

Consider an example in

which a hyperplane partitions 20 class 1 data points and 10 class 0 data points. 16 class 1 data points and 4 class 0 data points are assigned to the class 1 node, while 6 class 0 data points and 4 class 1 data points are assigned to the class 0 node. The Entropy of the class 1 node would be: $-\left(\frac{16}{20}\right) \log _{2}\left(\frac{16}{20}\right)-\left(\frac{4}{20}\right) \log _{2}\left(\frac{4}{20}\right)=0.7219$, while the Entropy of the class 0 node would be:

$$
-\left(\frac{6}{10}\right) \log _{2}\left(\frac{6}{10}\right)-\left(\frac{4}{10}\right) \log _{2}\left(\frac{4}{10}\right)=0.971 \text {. The Entropy for the hyperplane is }
$$

the weighted average of the Entropies of the child nodes: $\frac{20}{30} * 0.7219+\frac{10}{30} * 0.971=$ 0.805 .

\subsubsection{Receiver Operating Characteristic (ROC)}

The receiver operating characteristic (Metz, 1978) was originally developed by the US navy in World War II as a method for differentiating between signal noise and incoming enemy aircraft radar signals. 
ROC, when applied to classification methods, offers an alternative means of measuring the performance of a classifier. Accuracy can be misleading, especially in cases where class populations are highly imbalanced. When creating a classifier for a dataset with 990 class 1 data points and 10 class 0 data points, a classifier with $99 \%$ accuracy could be created by stating that all points are class 1 .

Rather than dealing with the total number of correctly or incorrectly classified data points, ROC deals with the number of correctly or incorrectly classified data points on a per class basis. The number of class 0 data points that are correctly classified is referred to as the number of true negatives (TN), and the number of class 0 data points that are incorrectly classified as class 1 data points is referred to as the number of false positives (FP). The number of class 1 data points that are correctly classified is referred to as the number of true positives (TP), and the number of class 1 data points that are incorrectly classified as class 0 data points is referred to as the number of false negatives (FN). This data can be represented in a compact form by using a table known as a confusion matrix. A confusion matrix is a table in which columns represent the class assigned to a data point by the classifier, and rows represent the actual class of the data points. Thus, a confusion matrix is presenting the following information:

Table 1: A confusion matrix

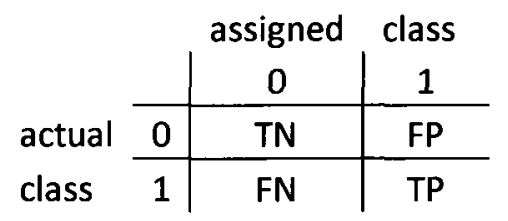

Using these numbers, true positive fraction (TPF), false positive fraction (FPF), true negative fraction (TNF), and false negative fraction (FNF) are defined as: 


$$
\begin{aligned}
& T P F=\frac{T P}{(T P+F N)} \\
& F P F=\frac{F P}{(T N+F P)} \\
& T N F=\frac{T N}{(T N+F P)} \\
& F N F=\frac{F N}{(T P+F N)}
\end{aligned}
$$

Working with these fractions eliminates the skewing of results that can be caused by imbalanced class populations. Going back to the example dataset that is made up of 990 class 1 data points and 10 class 0 data points, if a classifier classifies all points as class 1 , then the following fractions would be found: $\mathrm{TPF}=1, \mathrm{FPF}=1, \mathrm{TNF}=0, \mathrm{FPF}=0$. The $\mathrm{TNF}=0$ shines a spotlight on the information that accuracy glossed over: all class 0 data points were misclassified.

Using 4 fractions as a means of measuring the performance of a classifier is impractical. A single measure is preferred for use as a metric. Note that:

$$
\begin{aligned}
& T P F+F N F=\frac{T P+F N}{T P+F N}=1 \\
& T N F+F P F=\frac{T N+F P}{T N+F P}=1
\end{aligned}
$$

Therefore if one of TPF or FPF is known, the other can be computed; the same applies for TNF and FPF. Thus only two of the four fractions are needed.

Euclidean ROC (EROC) is one way of combining two of the required metrics into a single meaningful metric. EROC is best explained with an example. Consider again the dataset consisting of 990 class 1 data points and 10 class 0 data points. The 
performance of three different classifiers might be as shown in the following 3 confusion matrices:

Table 2: The performance of 3 different classifiers on one dataset

\section{Classifier 1}

\begin{tabular}{lr|r|r}
\multicolumn{1}{c}{} & \multicolumn{2}{c}{ asgn. } & \multicolumn{2}{c}{ class } \\
actual & 0 & 0 & 1 \\
\cline { 2 - 4 } class & 0 & 0 & 10 \\
\hline & 1 & 0 & 990
\end{tabular}
Classifier 2

\begin{tabular}{lr|r|r} 
& & \multicolumn{2}{c}{ asgn. } \\
& & 0 & 1 \\
\cline { 2 - 4 } actual & 0 & 5 & 5 \\
\cline { 2 - 4 } class & 1 & 190 & 800
\end{tabular}

Classifier 3

\begin{tabular}{lr|r|r} 
& & \multicolumn{2}{c}{ asgn. } \\
& & 0 & 1 \\
\cline { 2 - 4 } actual & 0 & 10 & 0 \\
\cline { 2 - 4 } class & 1 & 400 & 590
\end{tabular}

Recall that only one of (TPF,FNF) and one of (TNF,FPF) need be known. Suppose TPF and FPF are the fractions chosen for use, and are then plotted. The resulting plot appears as shown in Figure 6:

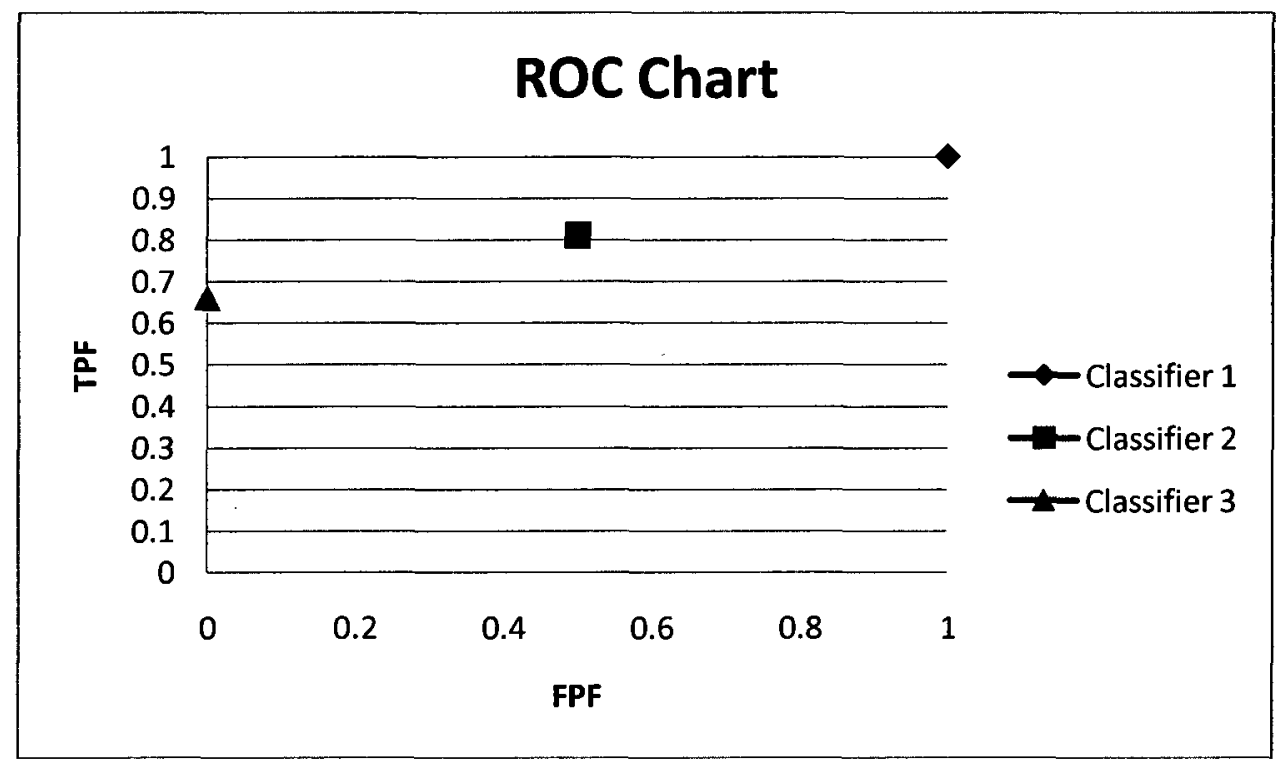

Figure 6: The performance of 3 classifiers in an ROC graph

In this chart, the TPF axis ranges from 0 to 1 , with 0 indicating that all positive data points have been misclassified, and 1 indicating perfect classification of these points. The FPF axis ranges from 0 to 1 , with 0 indicating that no negative data points have been misclassified, and 1 indicating that all negative data points have been misclassified. A perfect classifier would plot at $(0,1)$ on the ROC chart, and a perfect misclassification of 
all points would plot at (1,0). Euclidean ROC (EROC) (Bramer, 2007) is defined as being the euclidean distance of a point on the (FPF,TPF) plane to the $(0,1)$ point on the same plane. Since the $(0,1)$ point represents perfect classification, the closer a classifier's point in the plane is to $(0,1)$, the better, and therefore as low a value of EROC as possible is desired. Of the 3 classifiers plotted in Figure 6, classifier 3 is closest to the $(0,1)$ point on the ROC plane, and would thus be deemed the best performing classifier when using EROC as a metric.

\subsubsection{Cost Function}

A cost function (Metz, 1978) operates by assuming that the 4 different result types, TN, FP, FN, TP all have a cost associated with them. For example, if the binary classification tree is trying to diagnose the presence or absence of a given disease, a TP would have a cost associated with treating the disease, a FP would have a cost associated with subjecting a healthy patient to the treatment for the disease etc... There might also be an overhead, a base cost associated with performing the diagnostic test. Thus a cost function would appear as:

$$
\text { Cost }=c_{\text {overhead }}+c_{t p} * T P+c_{t n} * T N+c_{f p} * F P+c_{f n} * F N
$$

\subsubsection{Hyperplane Creation Methods}

\subsubsection{Univariate Hyperplane Creation Methods}




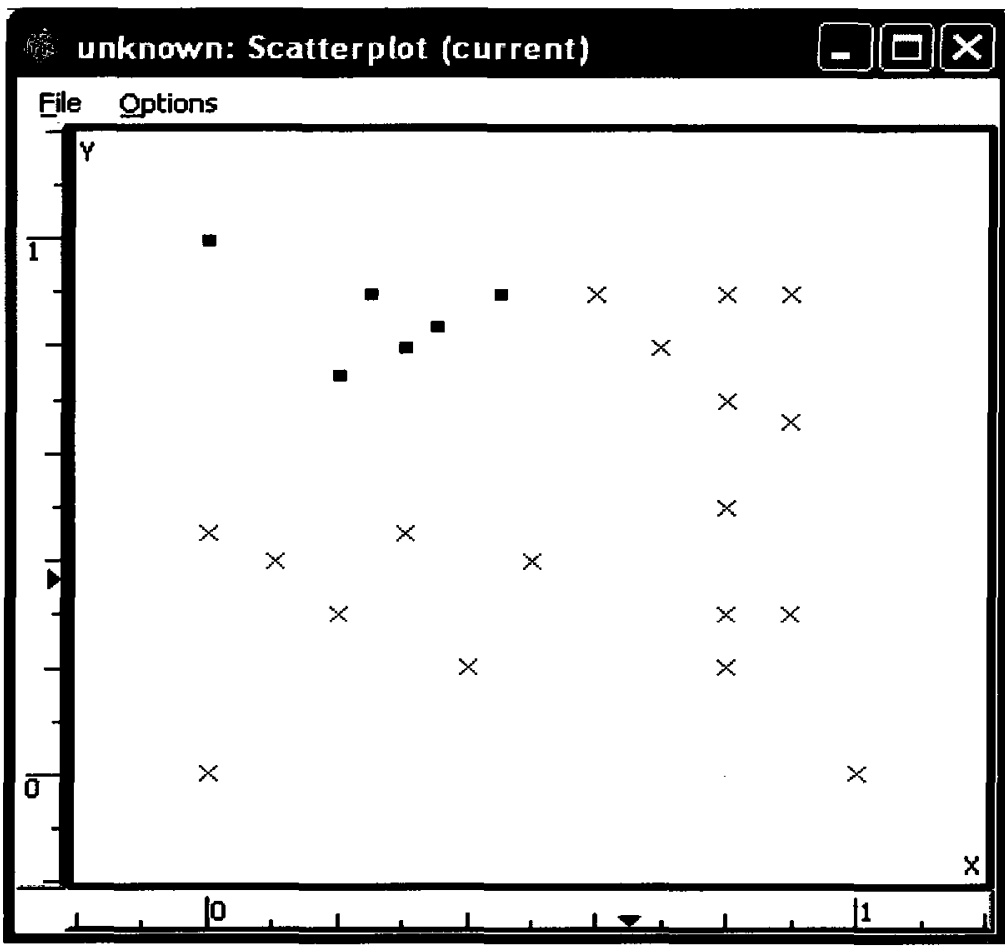

Figure 7: A sample data set

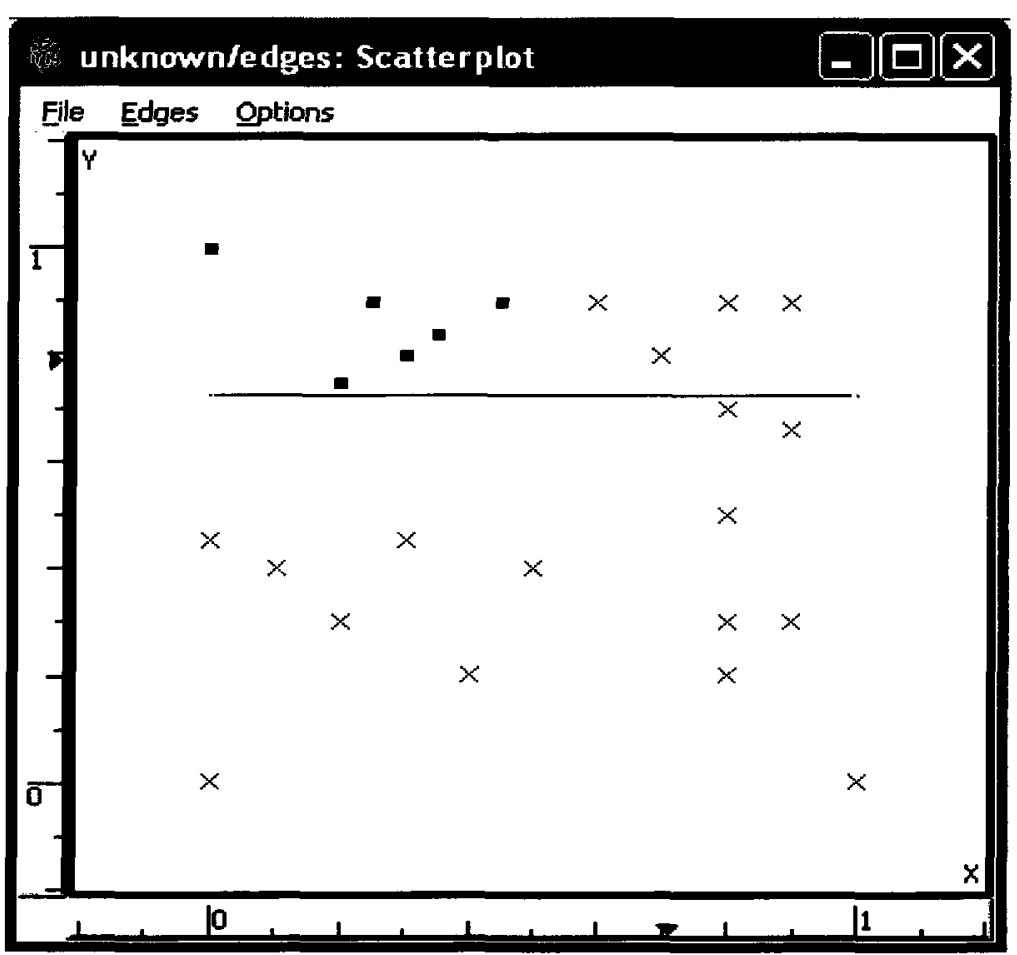

Figure 8: A first univariate split
Univariate

hyperplane placement

techniques were pioneered

by Breiman et al. (1984)

and Quinlan (1986), and

involve the creation of

hyperplanes that are one

dimensional. During the

tree creation process, a

decision is made at each

node regarding which

predictor (dimension)

yields the most information

about the data present in

that node. This predictor is

then used to create an axis-

parallel hyperplane to

further subdivide that data 


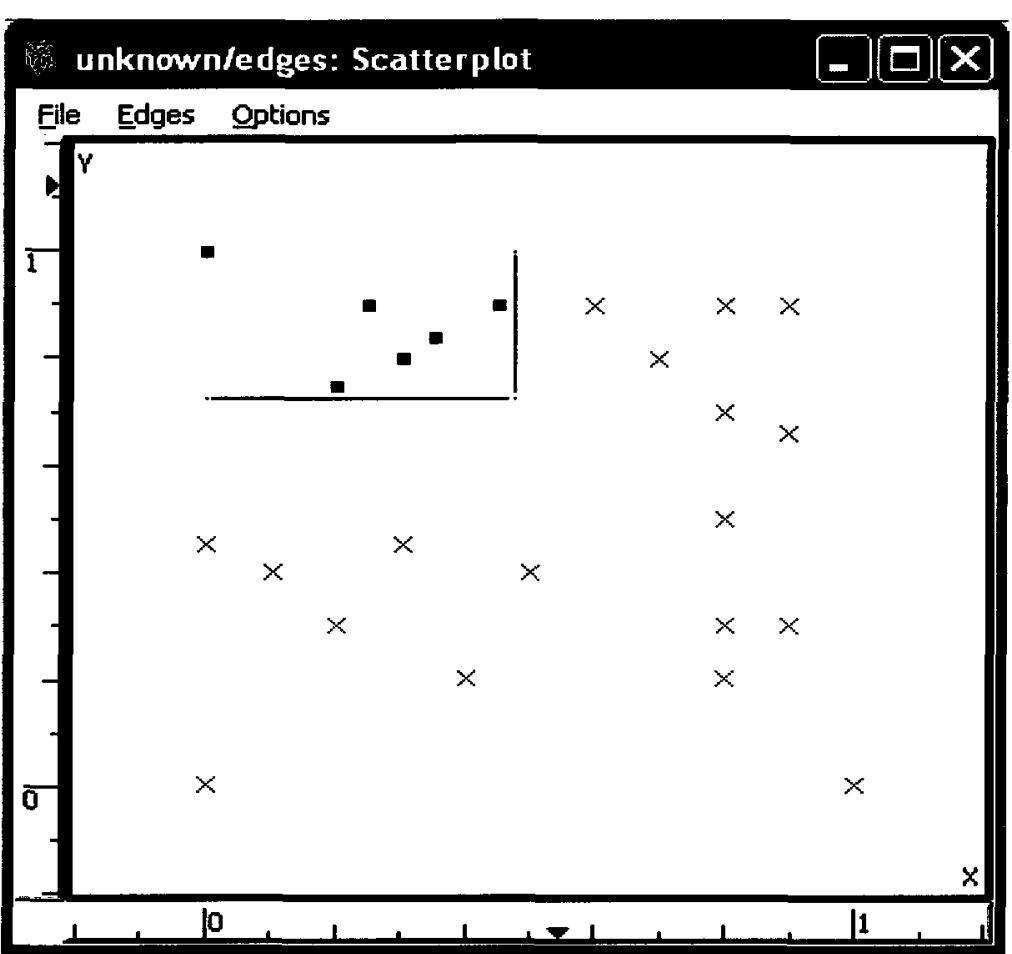

Consider the example shown in Figure 7.

Suppose that $\mathrm{Y}$ is the variable or dimension chosen for the first hyperplane. The first hyperplane then appears as shown in Figure 8.

$\mathrm{X}$ is then the next dimension or variable used Figure 9: The 2 univariate splits correctly classify all the data to create a second hyperplane. The data is now perfectly classified as shown in Figure 9. The graphical representation of this tree is as shown in Figure 10.

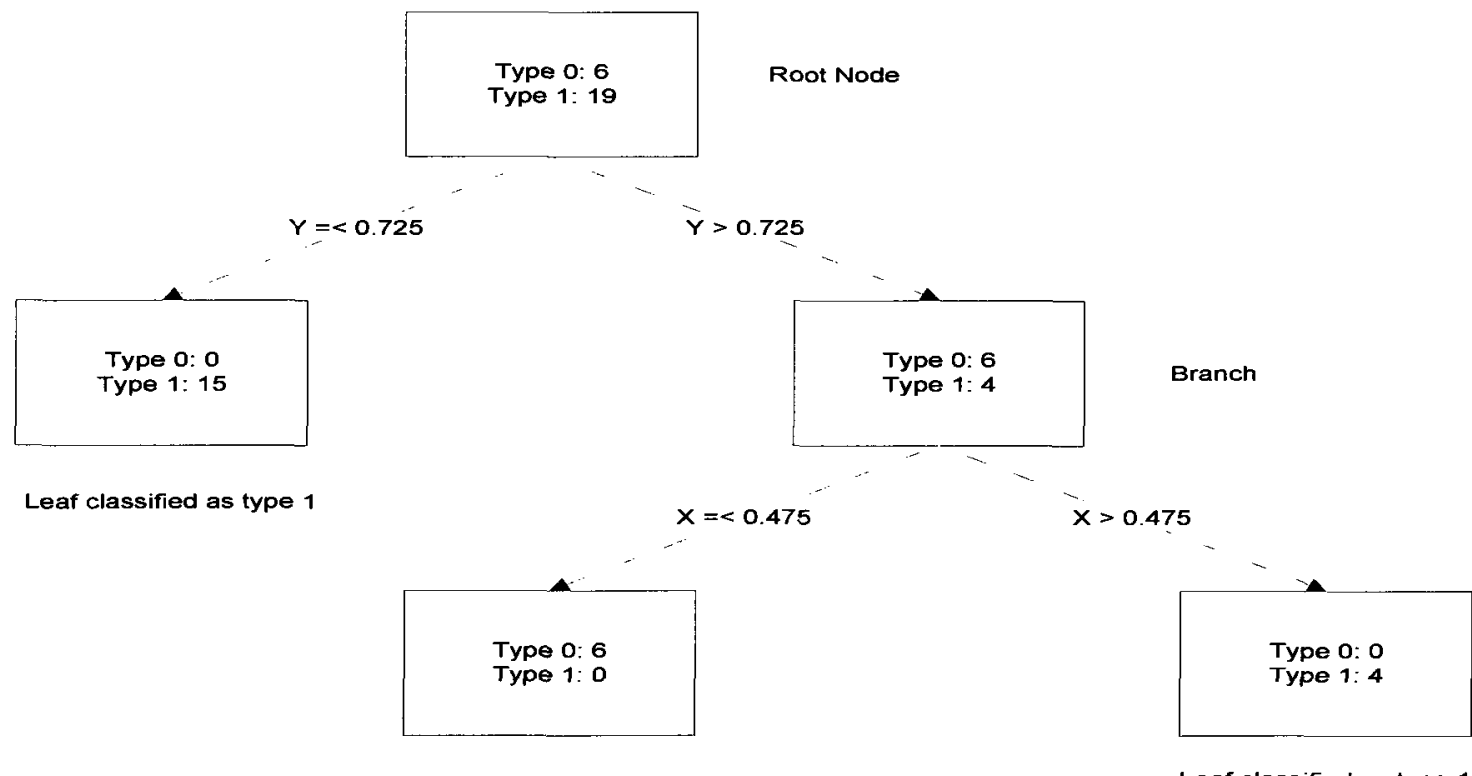

Figure 10: A univariate binary classification tree 


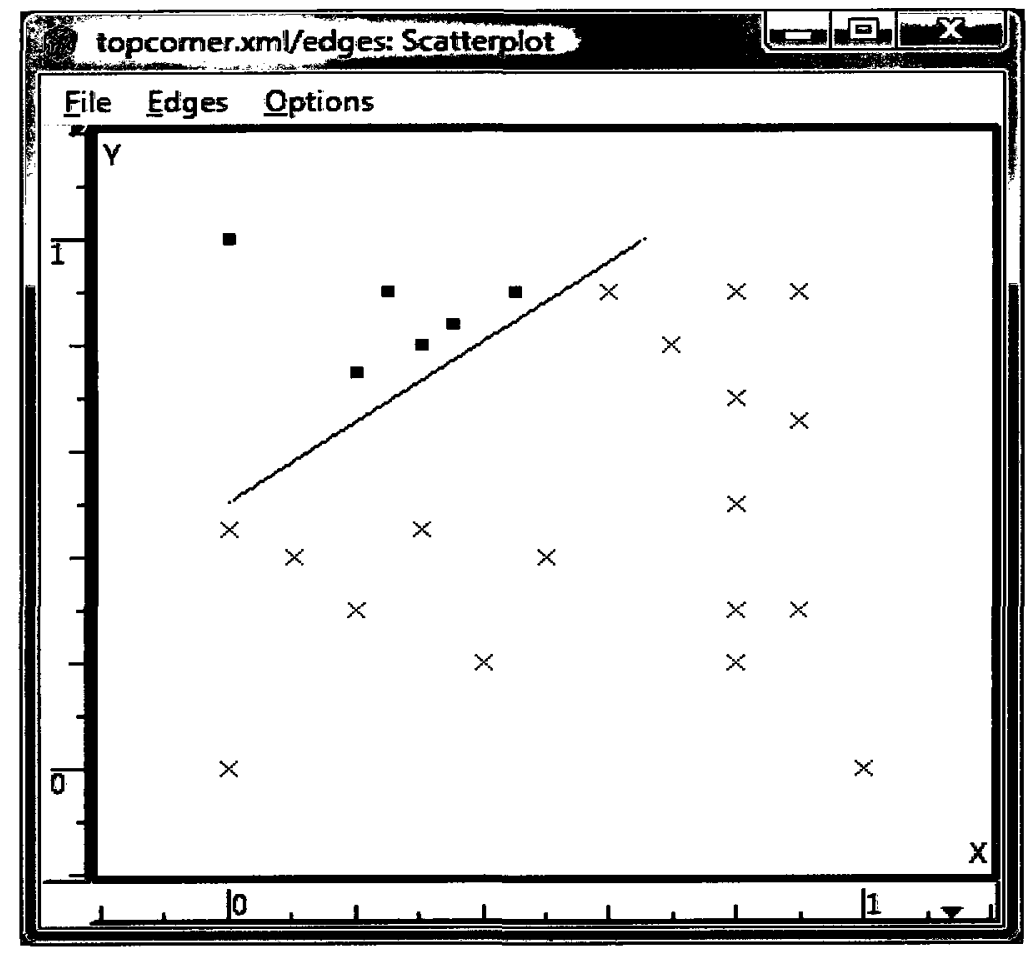

Figure 11: A single multivariate hyperplane classifies all the data correctly

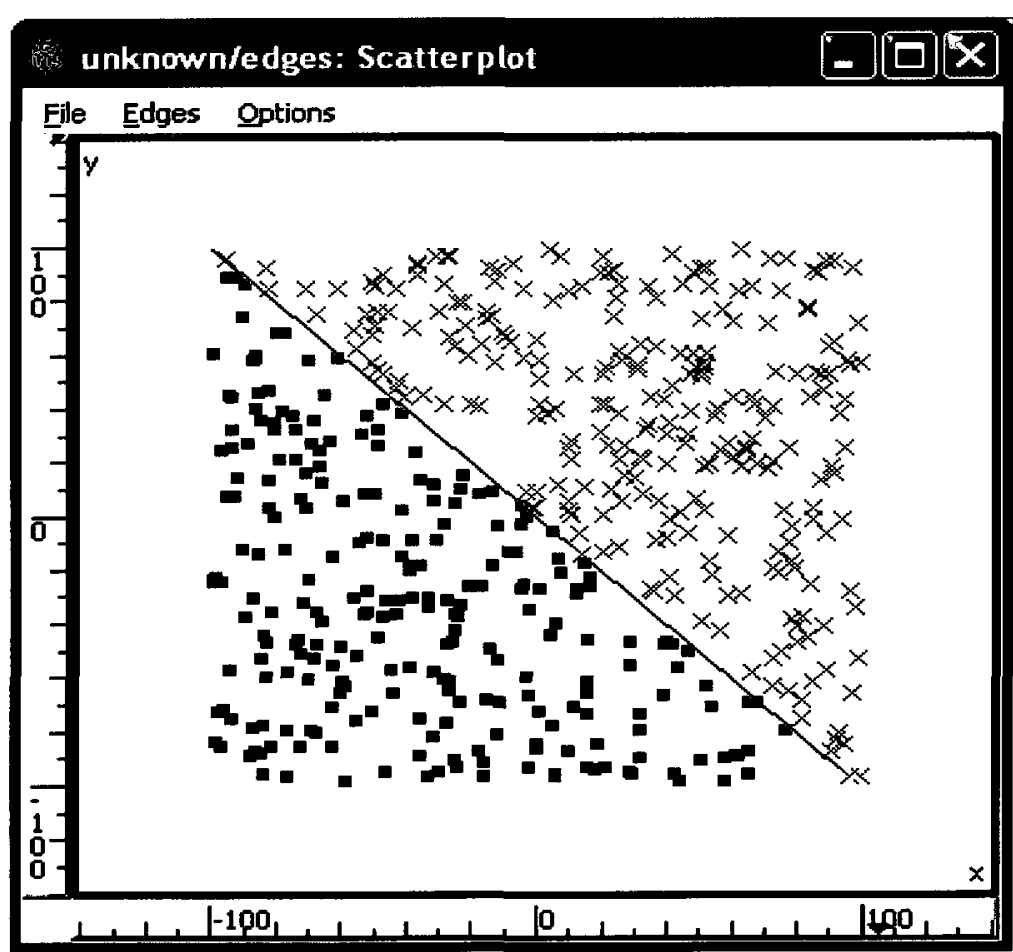

Figure 12: An example in which it would take hundreds of univariate hyperplanes in a staircase pattern to classify this dataset.
The previous

example can give some

insight into the main

problem with univariate

hyperplanes. In order to

classify the data, two

hyperplanes were needed.

Taking the same example,

and solving using

multivariate hyperplanes,

the data can be perfectly

classified using a single

hyperplane, as shown in

Figure 11.

In most cases,

information about a dataset

is not contained in a single

dimension, but in

combinations of

dimensions. Creating

univariate hyperplanes

prevents the tree learning algorithm from capturing information that is multi-dimensional. 
Figure 11 illustrates a minor problem, using two splits instead of one to classify data. In practice, hundreds of univariate hyperplanes are sometimes required to classify data that a single multivariate hyperplane could handle; Figure 12 is an example of such a case.

\subsubsection{Multivariate Hyperplane Placement Methods}

Multivariate hyperplanes, pioneered by Duda and Hart (1973) and by Breiman et al. (1984), allow the creation of hyperplanes that are linear combinations of several dimensions. The drawback to the use of multivariate hyperplanes is the significant increase in the size of the solution space. With univariate hyperplanes, every possible hyperplane can be examined for every dimension, allowing the tree creation algorithm to choose the best axis-parallel hyperplane at each and every node. The use of multivariate hyperplanes increases the number of possible hyperplanes to such an extent that examining all the different possible hyperplanes becomes practically impossible. A heuristic is needed, some method of generating a small set of good hyperplane candidates from which to choose.

Statistically Based Multivariate Hyperplane Placement Methods

CART-LC stands for CART with Linear Combinations, and was the first tree building algorithm to make use of multivariate hyperplanes (Breiman et al., 1984). This method finds an initial univariate hyperplane, and then iterates through the other dimensions making small random adjustments to the coefficients associated with each of those dimensions in an effort to find better multivariate hyperplanes. This process of adjusting the hyperplane stops when the benefit of such adjustments falls below an arbitrary threshold. 
QUEST (Quick Unbiased and Efficient Statistical Tree) was developed by WeiYin Loh and Yu-Shan Shih (1997). While Quest can be used to generate univariate hyperplanes, it also is capable of generating multivariate hyperplanes as well. Multivariate hyperplanes are decided upon by partitioning data, generating a large number of possible multivariate hyperplanes and then choosing the hyperplane with the best performance.

CRUISE (Classification Rule with Unbiased Interaction Selection and Estimation) was developed by Hyunjoong Kim and Wei-Yin Loh (2001). This tree creation process makes use of Linear Discriminant Analysis (LDA) in order to generate candidate hyperplanes. LDA is a statistical process that attempts to find linear combinations of predictors that will do the best job of separating two classes.

$\underline{\text { LP Based Multivariate Hyperplane Placement Methods }}$

Representing the hyperplane placement problem as an LP was first proposed in 1973 (Duda and Hart). Their method minimized the total distance of the misclassified points from the hyperplane.

For any dataset with $n$ dimensions, a hyperplane that separates the data will have $n$ variables and $n+1$ coefficients, with the hyperplane taking the form of:

$$
c_{1} x_{1}+c_{2} x_{2}+\ldots+c_{n} x_{n}+c_{n+1}=0
$$

If this hyperplane successfully classifies all the data points correctly then

for every class 1 data point specified by $\left(x_{1}, x_{2}, \ldots, x_{n}\right)$,

$$
c_{1} x_{1}+c_{2} x_{2}+\ldots+c_{n} x_{n}+c_{n+1} \geq 0
$$

and for every class 0 data point specified by $\left(x_{1}, x_{2}, \ldots, x_{n}\right)$,

$$
c_{1} x_{1}+c_{2} x_{2}+\ldots+c_{n} x_{n}+c_{n+1} \leq 0
$$


From this concept, we can construct a list of constraints (Chinneck et al., 2008), one for every data point. Each constraint stipulates that the associated data point must be above or below the hyperplane, depending on the class of the point. Thus the constraint for any given data point takes the form of:

- $c_{1} x_{1}+c_{2} x_{2}+\ldots+c_{n} x_{n}+c_{n+1} \leq 0$ for class 0 points

- $c_{1} x_{1}+c_{2} x_{2}+\ldots+c_{n} x_{n}+c_{n+1} \geq 0$ for class 1 points

where $x_{j}$ are known dimensions of each data point, and $c_{j}$ are the unknown variables which will eventually form the coefficients of the hyperplane.

For a dataset with $m$ points, there are $m$ constraints that must be satisfied. Any feasible solution to this LP will yield the coefficients for a hyperplane that successfully classifies all the data points.

Degeneracy is a case where all the data points in a dataset are placed on one side of the hyperplane. The use of the constraints just listed may result in a degenerate solution; one such solution that would satisfy all the constraints would be to set all the coefficients to be equal to zero. To help avoid this problem, a small positive constant $\epsilon$ is introduced into the constraints to act as a margin. The constraints now become:

- $c_{1} x_{1}+c_{2} x_{2}+\ldots+c_{n} x_{n}+c_{n+1} \leq-\epsilon$ for class 0 points

- $c_{1} x_{1}+c_{2} x_{2}+\ldots+c_{n} x_{n}+c_{n+1} \geq \in$ for class 1 points

- $c_{i}$ unrestricted in sign

In most cases, there is no single hyperplane that will classify all the data points correctly. How misclassified points are handled is the core of any LP based tree creation algorithm. Incorrectly classified points show up as violated constraints in the LP. In order to ensure that the LP passed to the LP solver has no infeasibilities, nonnegative 
elastic variables are introduced, one per constraint. If a constraint is for a type 0 point, the elastic variable is subtracted; otherwise the elastic variable is added. Thus it is now certain that any LP can be satisfied by adjusting the values of the elastic variables present. Thus for point $i$, the constraint now becomes:

- $c_{1} x_{1}+c_{2} x_{2}+\ldots+c_{n} x_{n}+c_{n+1}-e_{i} \leq-\epsilon$ for class 0 points

- $c_{1} x_{1}+c_{2} x_{2}+\ldots+c_{n} x_{n}+c_{n+1}+e_{i} \geq \in$ for class 1 points

- $c_{i}$ unrestricted in sign

- $e_{i} \geq 0$

These constraints that make use of elastic variables are referred to as elasticized constraints.

The goal is to have as few non-zero elastic variables as possible, since a non-zero elastic variable represents a violated constraint (and therefore a misclassified point). Minimizing the number of non-zero elastic variables creates an integer program, so the LP attempts to minimize the Sum of the Elastic Variables (SEV) instead:

Objective Function: Minimize $\sum_{i} e_{i}$ Where $e_{i} \geq 0$ for $\mathrm{i}=1 \ldots \mathrm{m}$

Now when an LP is solved, the solution will specify the coefficients of the separating hyperplane, and the hyperplane shows which data points are misclassified, and by how much (relatively speaking). This flexibility offers the opportunity to employ all sorts of heuristics to deal with misclassified points. The following example illustrates the LP representing an AND data set: 
Table 3: The AND dataset

\begin{tabular}{|c|c|c|}
\hline$x$ & $y$ & Output \\
\hline 0 & 0 & 0 \\
\hline 0 & 1 & 0 \\
\hline 1 & 0 & 0 \\
\hline 1 & 1 & 1 \\
\hline
\end{tabular}

A hyperplane in this 2 dimensional dataset takes the form:

$$
c_{1} * x+c_{2} * y+c_{3}=0
$$

The 4 data points introduce the following 4 constraints on the values of the coefficients of this hyperplane:

$$
\begin{gathered}
0 * c_{1}+0 * c_{2}+c_{3}-e_{1} \leq-\epsilon \\
0 * c_{1}+1 * c_{2}+c_{3}-e_{2} \leq-\epsilon \\
1 * c_{1}+0 * c_{2}+c_{3}-e_{3} \leq-\epsilon \\
1 * c_{1}+1 * c_{2}+c_{3}+e_{4} \geq \epsilon
\end{gathered}
$$

And the objective function is to minimize the sum of the elastic variables:

$\left(e_{1}+e_{2}+e_{3}+e_{4}\right)$

Assuming that $\epsilon=1$, the solution to this $L P$ is:

$c_{1}=2, c_{2}=2, c_{3}=-3, e_{1}=e_{2}=e_{3}=e_{4}=0$

And the equation of the hyperplane is:

$2 x+2 y-3=0$ 


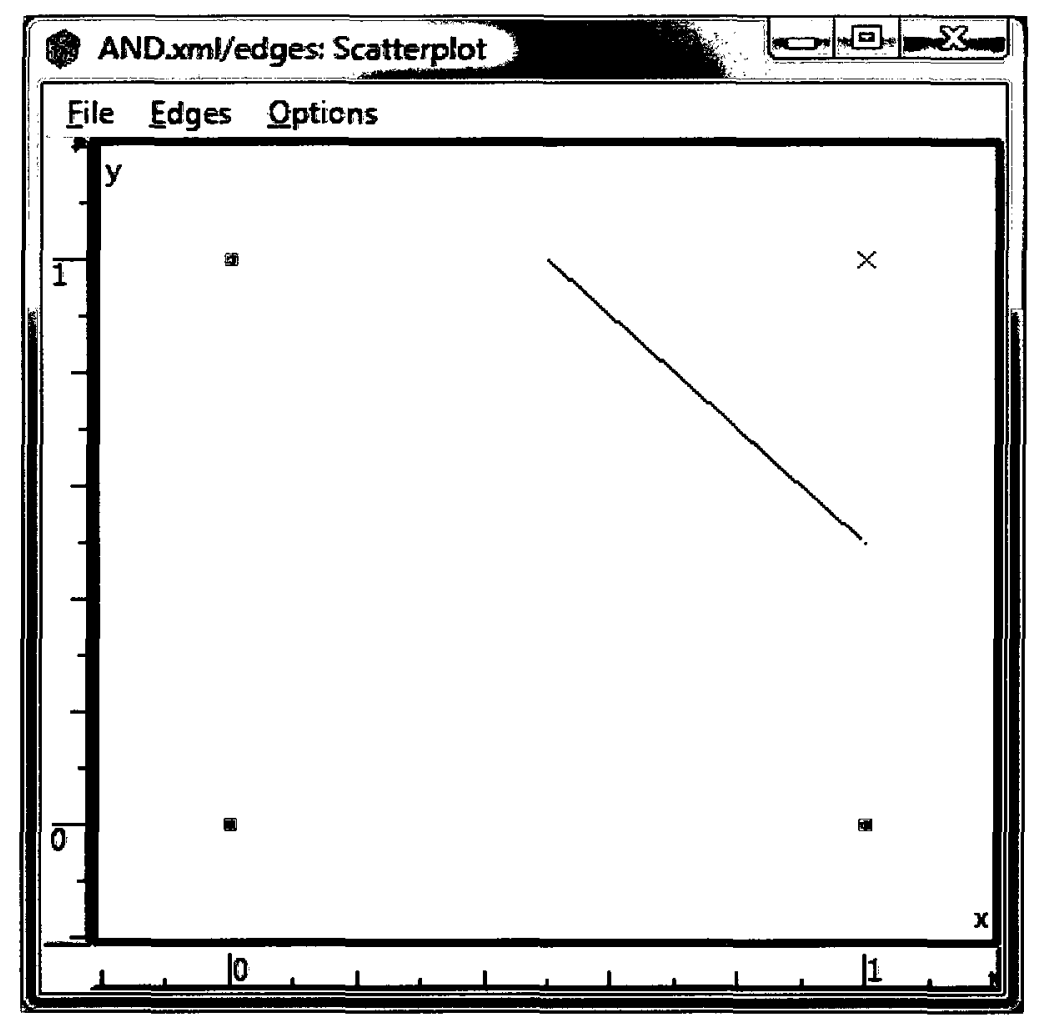

A plot of this dataset, with this hyperplane is shown in Figure 13.

Figure 13: The solution for the AND dataset

\subsubsection{Avoidance of Over-Fitting}

The purpose of a binary classification tree is to extract useful information about a given data point's class from its predictors.

Anomalies can be found in almost any dataset. Data points whose predictors were incorrectly measured, and data points whose predictors just don't conform to other predictors of the same class are examples of anomalies.

A good decision tree will successfully extract useful information that applies to most of the data points of a given class, while ignoring the misinformation that anomalies give. A bad decision tree creates hyperplanes that attempt to correctly classify the anomalies in a dataset. This behavior is known as over-fitting (Bramer, 2007). While this may result in a tree that performs well on its training dataset, this tree will likely perform poorly when presented with other data that was not used in training. 


\subsubsection{Stopping Criterion}

Even the most basic tree creation procedure must have some criterion in place to determine when to stop branching. The most basic tree construction methods will stop branching if one of the following two conditions is met (Quinlan, 1993):

1) All data points in a node belong to the same class

2) All data points have exactly the same predictors

Using only these criteria, the procedure will continue to branch a tree until all of the data is perfectly classified, except for those cases where two points of different class have exactly the same predictors. Using basic stopping criterion alone will not prevent over-fitting.

There are numerous rules that can provide more robust stopping behavior. Three examples of such rules are:

1) Maximum tree depth: Specify a depth to which a tree may expand. After that, the tree is terminated (Rokach and Maimon, 2002).

2) Minimum number of data points: For any given branch to be acceptable, each child node must have a minimum number of data points (Rokach and Maimon, 2002).

3) Minimum metric improvement: In order for a branch to be kept a certain improvement in metric must be gained, otherwise the branch is discarded. (Safavian and Landgrebe, 1991)

These rules help limit the growth of a decision tree, and thus prevent over-fitting. 


\subsubsection{Pruning}

Basic stopping criteria, described in Section 2.1.4.1, can result in fully expanded and over-fitted trees. If, after fully expanding a tree, the hyperplanes are re-examined and those that contribute to over-fitting are removed, then an acceptable tree is left. This process of removing hyperplanes is called pruning.

There are numerous pruning methods currently in use. Almost all fall into two basic categories (Esposito et al., 1997):

\section{Pruning By Using a Test Dataset}

These pruning methods require that a portion of the data be set aside as a test or pruning dataset. Once the tree is created using the training dataset, the resulting tree is then tested with the previously unseen test or pruning set. Generally speaking, the tree's performance on the training dataset will improve as the tree grows larger. The tree's performance on the test dataset will improve with tree growth only to a certain extent. After that limit has been reached, the test set's performance drops, due to the fact that the growing tree is now over-fitting itself to the training dataset. Breiman et al. (1984) provide an excellent example of this in Figure 14. 


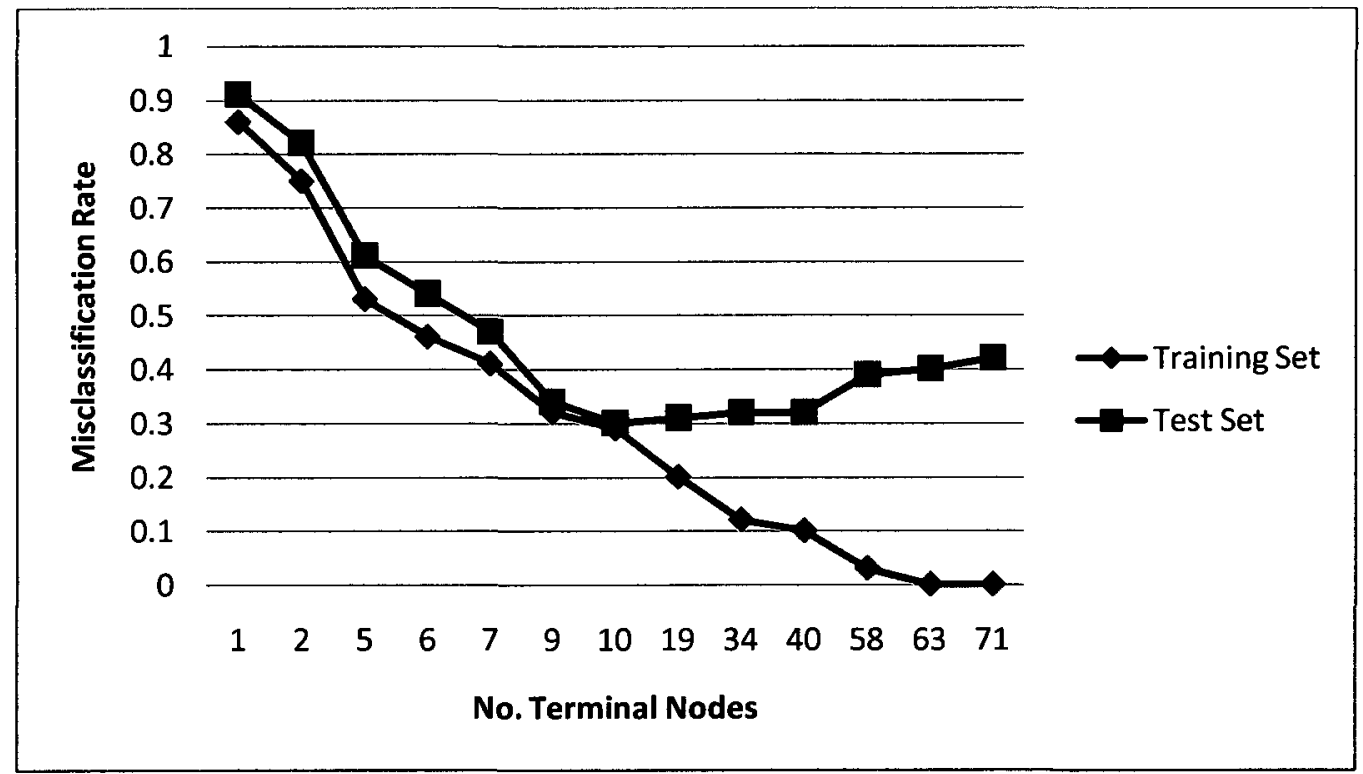

Figure 14: Test Set vs. Training Set Performance

The goal is to prune the tree back to the point where it performs optimally on the test dataset. The various methods that perform this sort of pruning distinguish themselves based on what measure they use for tree performance, and how they choose which nodes are candidates for pruning.

\section{Pruning Without Using a Test Dataset}

This method of pruning traverses through a fully branched tree and prunes branches whose addition results in a metric improvement that is below a specified threshold. Methods vary on what metric is used to decide whether or not to prune a branch, and in which direction to traverse the tree.

Esposito et al. (1997) provide an authoritative reference regarding decision tree pruning. A well written summary of these pruning methods is presented by Moghrabi (2003). 


\subsubsection{A Comparison of Pruning to Stopping Criteria}

Stopping criteria prevent over-fitting by halting tree growth during the tree creation process. When tree growth is halted depends on what stopping criteria are selected. The process of pruning takes place after a tree is fully grown, and re-examines the various hyperplanes in the tree to see if performance can be improved by the removal of certain hyperplanes.

Decision tree learning methods that make use of stopping criteria are faster due to the fact that tree size limitations are enforced when building the tree. Decision tree learning methods which employ pruning as a way of avoiding over-fitting take more time due to the fact that the tree is grown to its maximum size, and unwanted branches are then removed. The drawback to stopping criteria is that they may not terminate tree growth at the appropriate time. Judging appropriate parameters for criteria such as maximum tree depth, or minimum population counts is difficult. Misjudgment that allows a tree to grow too large, or cuts growth short, will have a negative impact on the performance of the tree (Esposito et al., 1997).

\subsection{Ten Fold Cross-Validation}

When creating a decision tree, if all the available data points are used in the training dataset, then testing is compromised since the test dataset will be a subset of the training dataset. Partitioning the dataset into a training set and a test set helps reduce this problem, but since the data points are randomly assigned to each set, results can still be skewed if the anomalous points are unevenly distributed.

$\mathrm{K}$-fold cross validation (Geisser, 1975) is a method of validating a classification procedure that partitions the data to allow for the creation of multiple training and test 
sets while maximizing the use of the data points available for tree creation. K-fold cross validation is performed by:

1) Splitting the dataset into K equally sized sub-datasets.

2) Each of these K sub-datasets will act as a test dataset.

3) The training dataset for each of the $\mathrm{K}$ test datasets is comprised of the remaining K-1 sub-datasets.

4) Train $K$ classifiers, using 1 training/test dataset pair per classifier

The results of the $\mathrm{K}$ classifiers are then averaged, and the variances are calculated to give a very good idea of the overall performance of a classifier.

\subsection{K Nearest Neighbour Classification}

When attempting to classify an unknown sample, the K Nearest Neighbour (also referred to as $K N N$ ) method examines the class of the $\mathrm{K}$ closest neighbours in the training set and assigns the sample the same class as the majority of its $\mathrm{K}$ closest neighbours from the training set (Cover and Hart, 1967). 


\section{A Review of the State of the Art}

When it comes to hyperplane placement in commercial data classifiers, development has been somewhat stagnant for a long time. CART, produced commercially by Salford Systems (2009), and See5 (Quinlan, 2002) produced commercially by Rule Quest, rely on the same basic hyperplane placement methods that their original creators described two decades ago.

Research into hyperplane placement methods in the academic community has been quite broad in scope, with some researchers focusing on developing hyperplane placement methods that are highly tailored towards a specific application (Lazaridès et al., 1996), while other researchers focus on developing methods that can be generally applied. The review of the state of the art will focus on hyperplane placement methods that are intended for broad general use, since the methods proposed in this thesis are also intended for general use.

\subsection{Homogenous Hyperplane Placement}

A homogenous hyperplane placement method is one that employs the same hyperplane placement algorithm in all situations.

\subsubsection{Univariate Hyperplane Placement}

Quinlan has produced numerous software packages for tree creation over the years: ID3 (Quinlan, 1986), C4.5 (Quinlan, 1993), and See5 (Quinlan, 2002). The hyperplane placement method has remained the same; univariate splits are created using entropy as a measure of a hyperplane's performance. 


\subsubsection{Multivariate Hyperplane Placement}

Cart (Breiman et al., 1984) has a commercial version produced by Salford Systems. The latest version, Cart 5 (Salford Systems, 2009), supports 7 univariate and one multivariate hyperplane placement algorithm. The univariate methods are applied by determining which dimension a split should be made on, and then finding the best split. The 7 univariate methods use different criterion for determining what the "best" split is. The multivariate hyperplane placement method is referred to as Cart-LC and is described in Section 2.1.3.2.

Chinneck (2009) has made numerous additions to the basic LP based hyperplane placement method described in 2.1.3.2. These modifications include:

1) Introducing an algorithm that uses the basic LP in an iterative process which attempts to create a hyperplane that misclassifies the fewest data points possible.

2) Implementing a heuristic to speed up the iterative process (Chinneck, 2009).

3) Introducing alterations to the iterative process to allow for the promotion of metrics other than accuracy (Chinneck, 2009).

This iterative approach to hyperplane placement forms the basis for the algorithms that are presented in this thesis. The algorithm for this process is detailed in Algorithm 1. 
INPUT: A set of data points, converted to elasticized linear constraints

1) Following the procedure outlined in Section 2.1.3.2, create a list of elasticized constraints

2) Solve the elasticized LP

3) If $\mathrm{SEV}=0$ then exit

4) Construct a list of candidate constraints for removal in decreasing order of merit

5) For each candidate constraint

a. Temporarily remove the candidate constraint

b. Solve the new LP and note the new SEV

c. Reinstate the removed candidate constraint

6) Permanently remove the candidate constraint whose removal yielded the greatest reduction in SEV

7) Go to step 2

OUTPUT: A hyperplane that misclassifies the fewest data points.

Algorithm 1: Chinneck's (2009) most recent LP based hyperplane placement algorithm

In the basic hyperplane placement method, the list of data points that are

candidates for removal is comprised of all those data points whose constraints are

violated or tight. A tight constraint is one which has a non-zero dual price in the solution

to the LP. Modifying the hyperplane placement in this algorithm is a matter of putting

restrictions on which data points can be candidates for removal. Chinneck (2009)

introduces numerous hyperplane placement methods using this technique; of particular

interest is a pure cut hyperplane placement algorithm. A pure cut hyperplane placement

is a hyperplane placement method which attempts to separate the largest homogeneous

group of data points that can be found. Chinneck (2009) achieves a pure cut hyperplane

placement by making a slight modification to Algorithm 1 . In order to create a pure cut

hyperplane, Step 4 in Algorithm 1 is modified such that only constraints belonging to the 
class that the pure cut hyperplane is to separate may be added to the list of candidate constraints for removal.

\subsubsection{Boosting}

Boosting (Schapire, 1989), is a heuristic that can be applied to homogenous hyperplane placement methods. The process of boosting involves creating numerous decision trees. After the first decision tree is created, the training set used to create this tree is weighted, giving greater weight to the data points that were misclassified by the decision tree just created. This weighted training set is then used to create a second tree, after which the training set is re-weighted, and a third decision tree is created etc...

Giving greater weight to a data point decreases the likelihood that it will be misclassified by a tree creation algorithm, since the greater weight will result in a lower metric being scored when that point is misclassified. Once $n$ trees have been created, classification of data is then done by having each of the $n$ trees classify a given point and then assigning the class arrived at by the majority of the trees to the data point in question.

Boosting is currently employed by commercial decision tree software packages such as C4.5 (Quinlan 1993), and See5 (Quinlan 2002).

\subsection{Heterogeneous Hyperplane Placement}

A heterogeneous hyperplane placement method is one that has more than one hyperplane placement algorithm that it can choose to make use of when placing a hyperplane.

Moghrabi (2003) explored the possible benefits of creating heterogeneous binary classification trees. A set of 9 policies that specified how to generate heterogeneous 
binary classification trees were created. 8 policies operated by creating numerous greedy candidate hyperplanes at any given node, and then choosing from among them. Different policies made use of different criteria to choose which candidate hyperplane would be kept. The $9^{\text {th }}$ policy created 8 trees in parallel, one for each of the 8 other policies, and would then keep the best performing tree. This policy will be referred to as "Moghrabi's best" since it takes the best performing of the other 8 policies as its own.

Ling and Sheng (2005) propose a hybrid classifier that combines a greedy, though cost sensitive, decision tree with a cost sensitive Naïve Bayes Classifier in an effort to achieve the best overall results. The decision tree, which uses univariate splits, is used to select from a group of tests, and the Naïve Bayes is used to actually classify the samples.

SMILES (Ferri-Ramírez et al., 2002), is a tree creation method that builds an initial tree with several OR nodes. An OR is actually a group of several candidate nodes, each of which is fully branched as a possible continuation of the tree. Once the initial tree is built, the OR nodes are all revisited, and the candidate nodes that result in a better overall tree are kept. Different candidate nodes are created by using a greedy hyperplane placement algorithm, but making use of different metrics.

\subsection{Non-greedy Tree Creation}

So far, no papers have been found that document non-greedy hyperplane placement methods.

There are a few tree creation algorithms in which a tree is created using greedy hyperplane placement methods. These hyperplanes are then modified to improve the overall performance of the tree, and this modification changes these hyperplanes from being greedy hyperplanes to being non-greedy hyperplanes. 
GATree (Athanassios and Dimitrios, 2001) is a tree creation method in which an initial greedy tree is created, and then a Genetic Algorithm is applied to alter the solution towards a better overall result.

GTO, created by Kristin Bennet (1994), generates a fully grown greedy tree, using a similar greedy LP based hyperplane placement method as Chinneck (2009). Once the greedy tree is fully grown and pruned, a second LP is formulated to take the tree and modify its branches in an effort to produce a better over-all tree. 


\section{Creating Better Binary Classification Trees}

\subsection{Motivation}

The motivation for designing better hyperplane placement algorithms is well illustrated by the graphs shown in Figure 15.
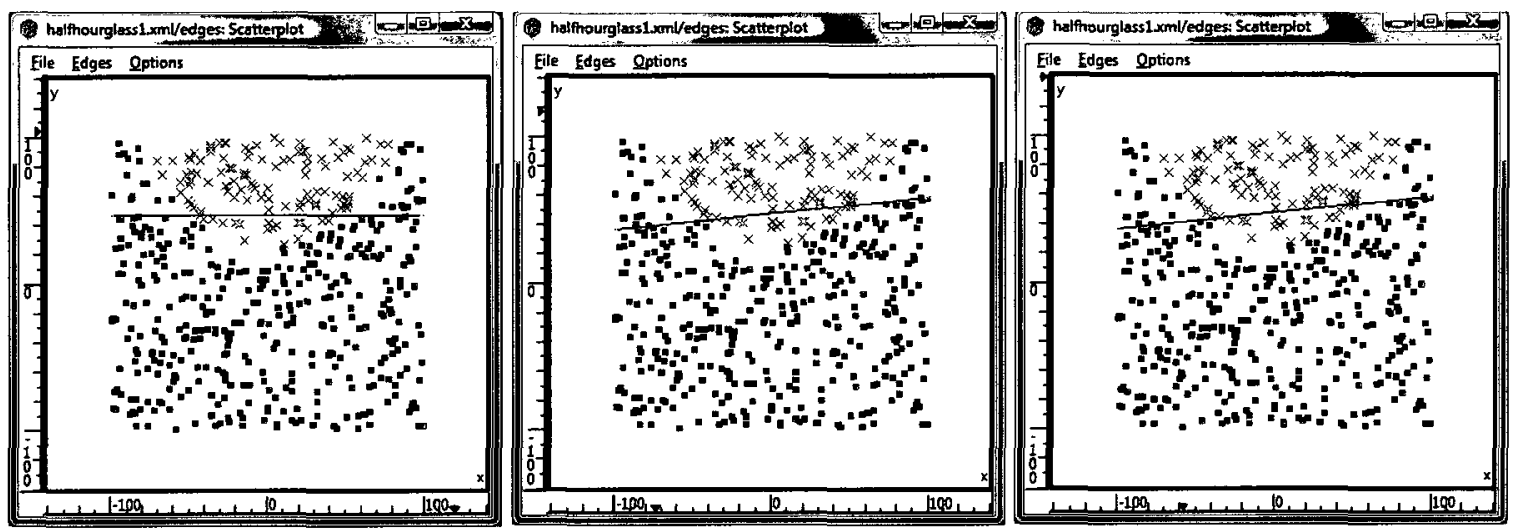

Figure 15: The first hyperplane placed using 3 different greedy algorithms: Accuracy, Gini, and Entropy

These graphs show the first hyperplane placed by three different greedy hyperplane placement algorithms on the synthetic dataset halfhourglass. The initial branches have all misclassified a group of class " $x$ " data points, and a greedy hyperplane placement method will have no way of placing additional hyperplanes that will result in these points being reclassified correctly.

The problem with greedy algorithms is that they maximize locally, not globally. When placing a hyperplane, a greedy algorithm works on the assumption that there will be no further branching performed, and therefore that all trade-offs in terms of which points are classified correctly and incorrectly need to be made immediately. 


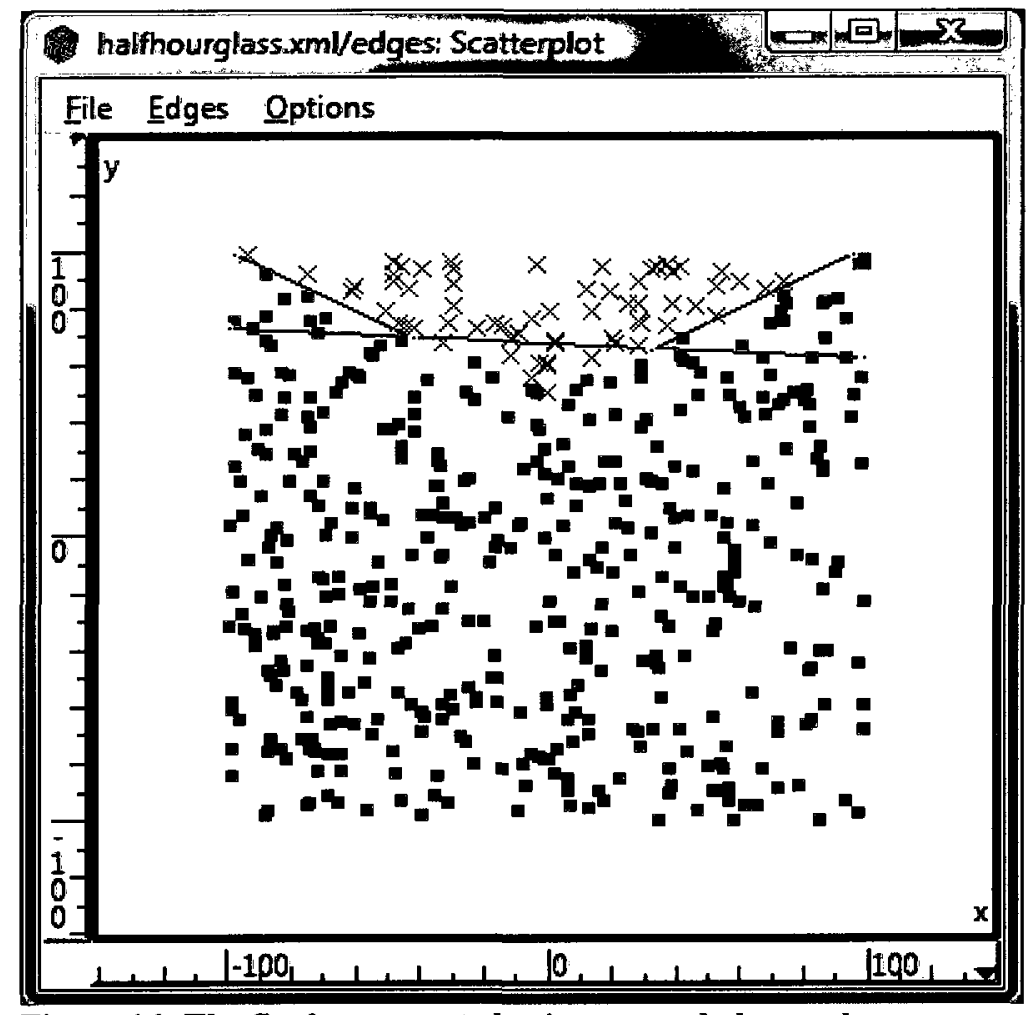

Figure 16: The final tree created using a greedy hyperplane placement method which maximizes for accuracy.
Figure 16 shows the final tree created using a greedy hyperplane placement method that maximizes for accuracy. The greedy algorithm was unable to reclaim that initial triangle of class " $x$ " points that were misclassified by the placement of the first hyperplane (shown in

Figure 15).

This example highlights the need for non-greedy hyperplane placement methods that avoid this pitfall.

\subsection{Problem Statement}

The goal of this thesis is to demonstrate that non-greedy hyperplane placement methods, used in heterogeneous tree construction policies, lead to the creation of better binary classification trees. New non-greedy hyperplane placement methods, and new heterogeneous policies for their application, will be developed. The new methods will be empirically compared to homogeneous greedy methods.

The various tree creation methods discussed in Chapter 1 , and the hyperplane placement methods they use, can be classified into three groups: 
- Homogenous Trees created using greedy hyperplane placement methods

- Heterogeneous Trees created using greedy hyperplane placement methods

- Homogeneous Trees created using greedy hyperplane placement methods that are later modified into non-greedy trees.

None of the literature reviewed suggests that anyone has attempted to create decision trees using non-greedy hyperplane placement methods.

\subsection{Overview of the Research}

The thesis research focuses on three main areas:

\section{Combinations of Greedy Hyperplane Placement Methods}

Accuracy, Gini, Entropy, EROC and Cost Functions are existing hyperplane placement methods that have all been implemented. Research with these methods focuses on creating policies that will choose which of these hyperplane placement methods to apply at a given node in order to create a better overall tree.

\section{Non-Greedy Hyperplane Placement Methods}

This research focuses on the creation of branches that don't maximize for any given metric. Rather an attempt is made to place branches that will produce the best overall tree. The thesis develops non-greedy methods that either:

1) Separate out a completely homogeneous or a completely heterogeneous zone of points.

2) Focus on a subset of data points that represents a single linear or near-linear interface between different zones. The interface could be between two homogeneous groups of data points, or between a homogeneous and a heterogeneous group of data points. 


\section{$\underline{\text { Policies }}$}

Policies are rule sets which specify what hyperplane placement technique should be used at a given node. Policies which allow combinations of multiple greedy methods, multiple non greedy methods, and even combinations of greedy and non greedy methods have been studied. The prime goal is to find a good way of selecting which hyperplane placement method should be used at any given point in time. 


\section{New Methods}

\subsection{New Non-Greedy Hyperplane Placement Methods}

The idea behind the non-greedy hyperplane placement methods developed here is to identify zones of intersection, either between two homogenous groups of data points, or between a homogenous group and a heterogeneous group.

\subsubsection{Mixed Zone Cut}

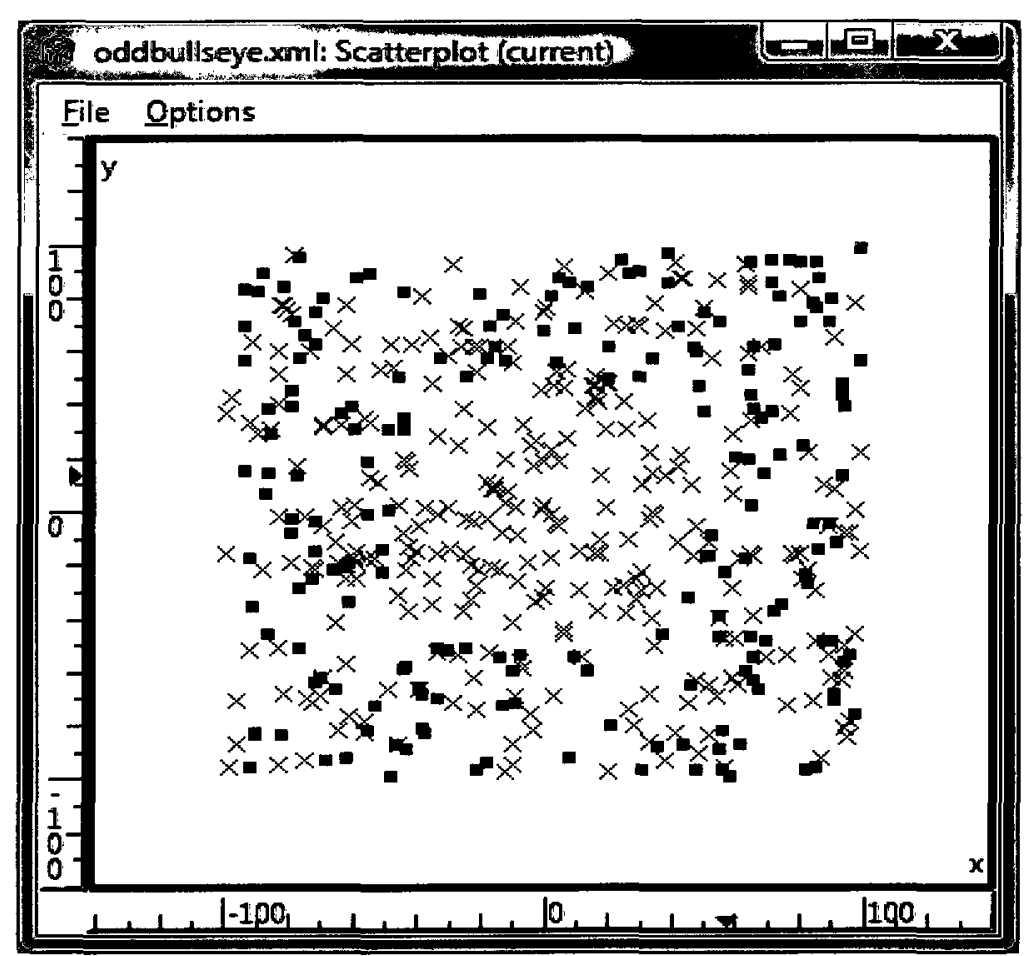

It is conceivable

that the outer edges of a

dataset or data subset could

be comprised entirely of a

mixed zone of data points.

Attempts to place a greedy

hyperplane result in

degeneracy and attempts to

place a pure cut hyperplane

Figure 17: The MixedBullseye dataset

also fail. The MixedBullsEye dataset illustrates this idea (Figure 17): This dataset

highlights the need for a hyperplane placement method that separates a mixed zone of data points from a pure zone. Such mixed zones can be further subdivided later. Once the mixed zones in this example are removed, there remains a homogenous center which can be classified as class 1 data points. 
In order to place a hyperplane that separates a group of mixed-zone data points from the rest of the dataset, the dataset must be transformed. Instead of separating class 0 and class 1 data points, the hyperplane now needs to separate mixed-zone data points from pure zone data points. This is still a binary classification problem. To determine if a data point is considered to be in a mixed zone, its' $\mathrm{K}$ nearest neighbours are examined. A data point which has a neighbour of the opposite class is considered to be in a mixed zone, a data point whose neighbours are all of the same class is not. All points considered to be in a mixed zone are reassigned to be class $M$ for mixed, all points in homogenous

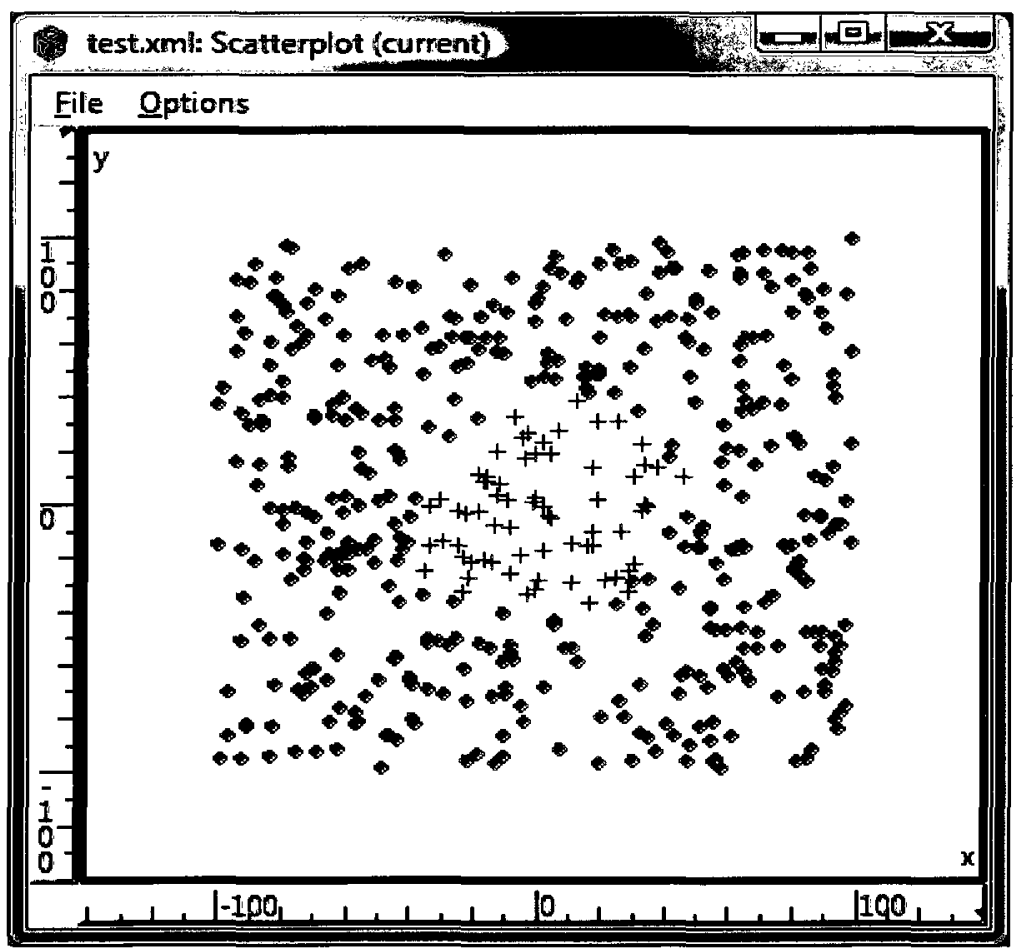

regions are considered to be class $P$ for pure. This transform, created using a $\mathrm{K}$ of 10 , results in a new dataset, shown in Figure 18.

\section{In Figure 18, a}

circle represents a class $M$ data point, and a "+" symbol represents a class $P$ data point.

Figure 18: The transformed dataset. "+" symbols represent class $P$ data points, and circles represents class $M$ data points 


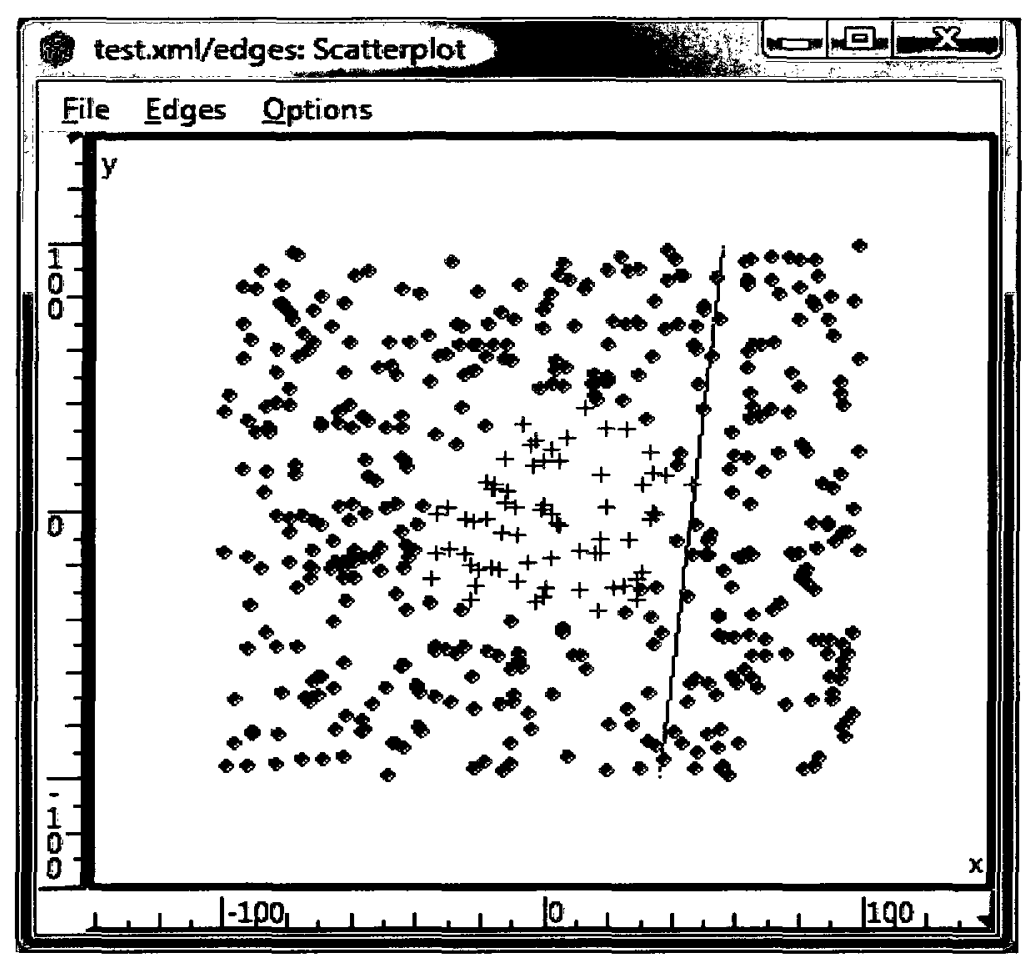

Figure 19: A pure cut taken on the transformed dataset

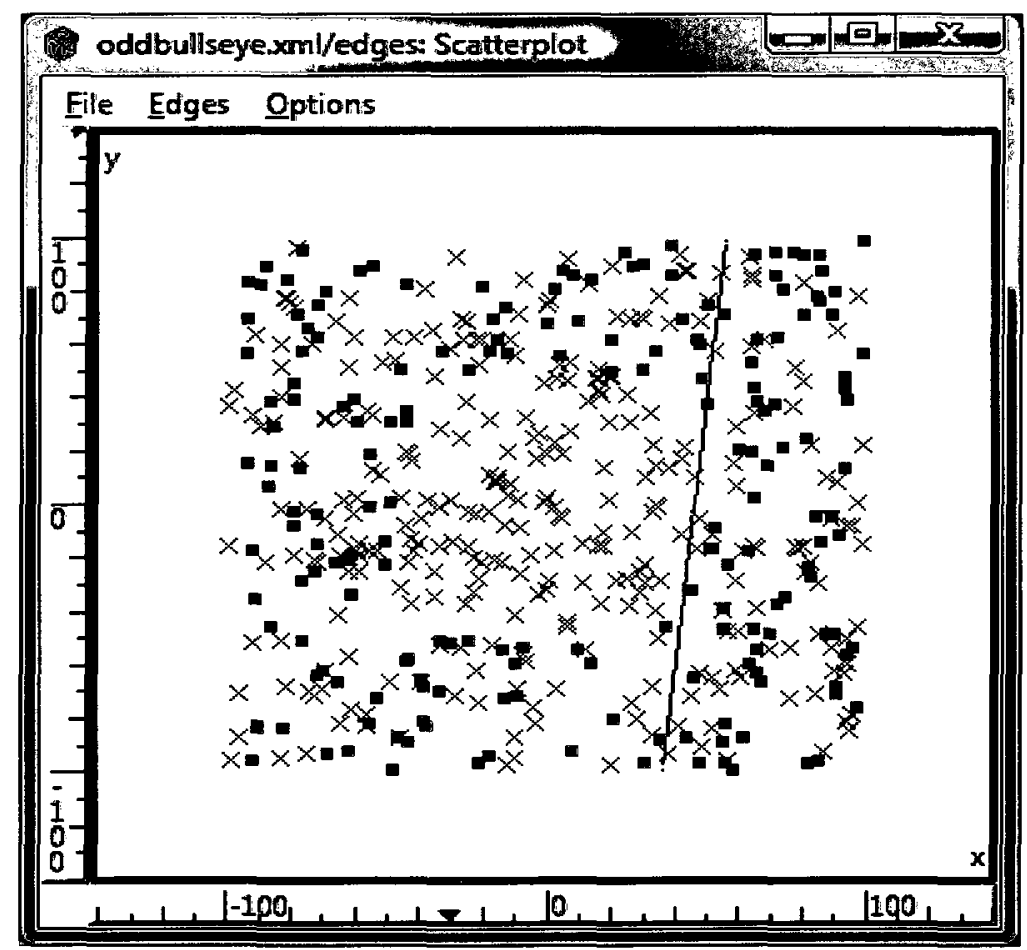

Figure 20: The same hyperplane seen on the original dataset
Now if a pure cut

hyperplane is created for

this transformed dataset, it

actually separates a group of

mixed zone data points from

a group of pure data points.

This pure cut

hyperplane is shown in

Figure 19, on the

transformed dataset.

\section{Placing the}

hyperplane just created on

the original dataset shows

that it has successfully

shaved off a mixed zone

from the rest of the dataset

(Figure 20). 


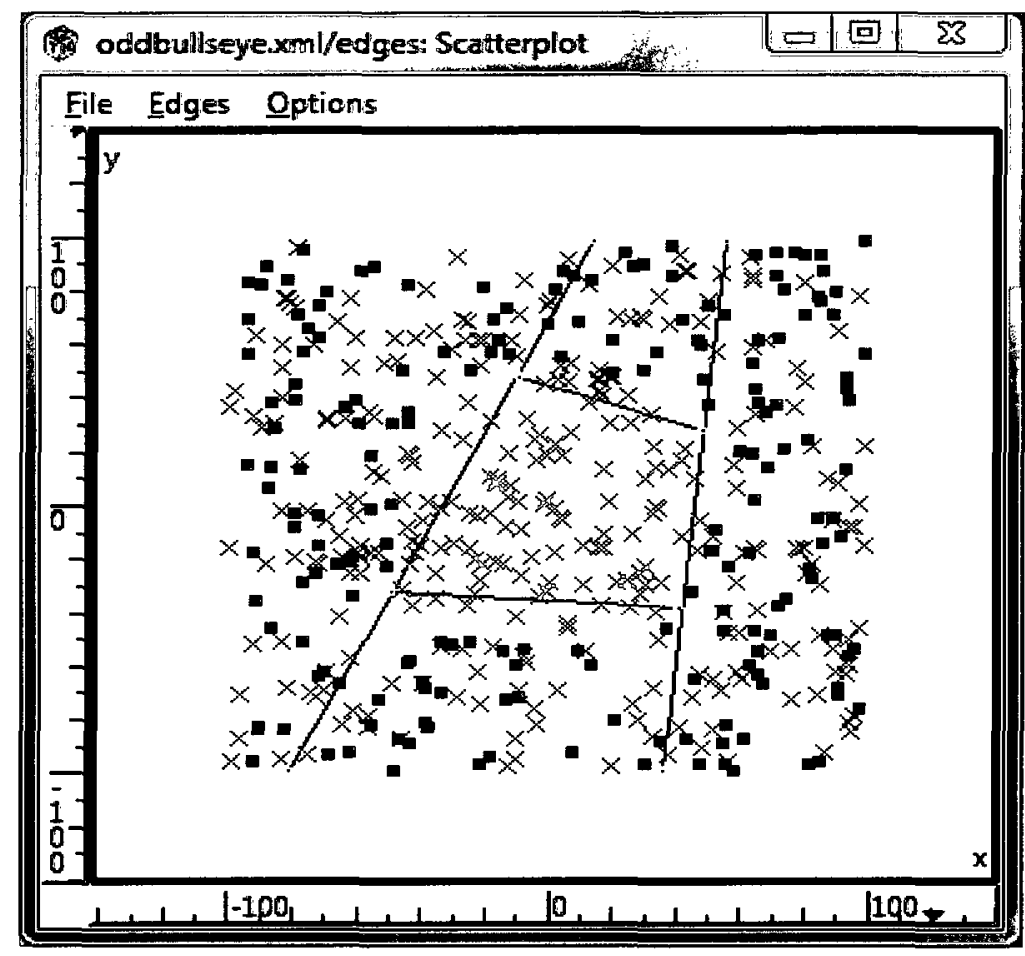

Do this three more

times and the homogenous

region of class 1 data

points is separated out from

the rest of the dataset, as

shown in Figure 21.

Pseudo code for

placing a mixed zone

separating hyperplane is

Figure 21: After 4 mixed zone cuts, the homogenous group of class 1 data points has been isolated.

shown in Algorithm 2.

INPUT: A dataset consisting of data points from two distinct classes: $\mathrm{A}$ and $B$

1) For each data point in the data set

a. Examine the $\mathrm{K}$ nearest neighbours

b. If any of those neighbours are of the opposite class, add this data point to the Transformed dataset as a class $M$ point. Otherwise add it to the Transformed data set as a class $P$ point

2) Place a pure cut on the Transformed subset.

OUTPUT: A hyperplane separating out a mixed zone of data points.

Algorithm 2: Mixed Zone Hyperplane Placement

\subsubsection{Edge Following (EF) Hyperplane Placement Methods}

The Edge Following Hyperplane Placement methods developed here attempt to

find regions where two different groups of data points meet, and then attempt to place a hyperplane through this region. The term "different groups" may refer to two 
homogenous groups of different class type, or a homogenous group and a heterogeneous group. The EF methods that deal with two groups of homogenous data points are called EF Class Isolation Pure Cut, and EF Class Isolation Greedy Cut. The Edge Following methods that deal with a homogenous group and a heterogeneous group of data points are called EF Mixed Isolation Pure Cut, and EF Mixed Isolation Greedy Cut.

Edge Finding methods offer an intuitive hyperplane placement method that differs from pure cut methods and greedy methods. Rather than trying to optimize for a given metric or trying to shave off a large homogenous or heterogeneous region of data, the Edge Finding methods attempt to place a hyperplane along the longest edge of intersection in the dataset being examined. The halfhourglass dataset will be used to illustrate this idea.

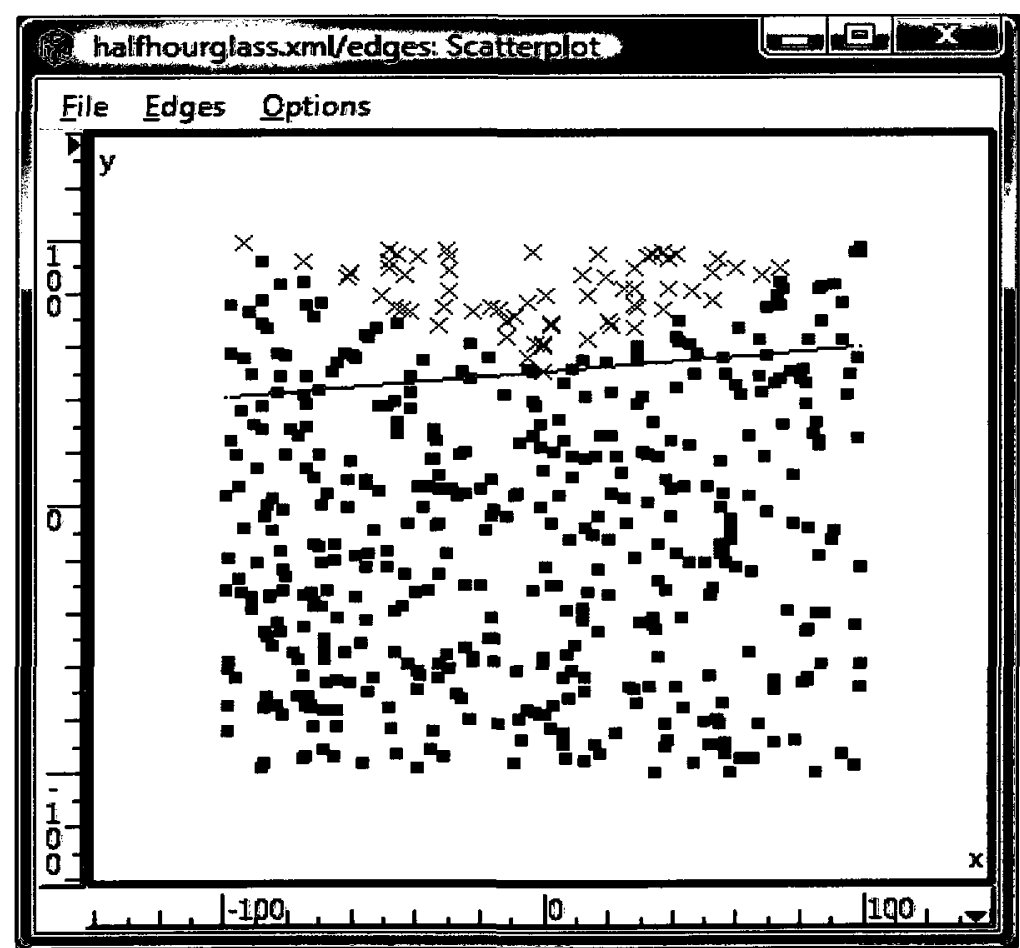

Figure 22: The first hyperplane placed by a pure cut hyperplane placement method
Section 4.1

illustrates how poorly

greedy hyperplane

placement methods do

when attempting to classify

this simple dataset. A pure

cut hyperplane placement

method performs better.

The first hyperplane

appears as shown in Figure 22. 


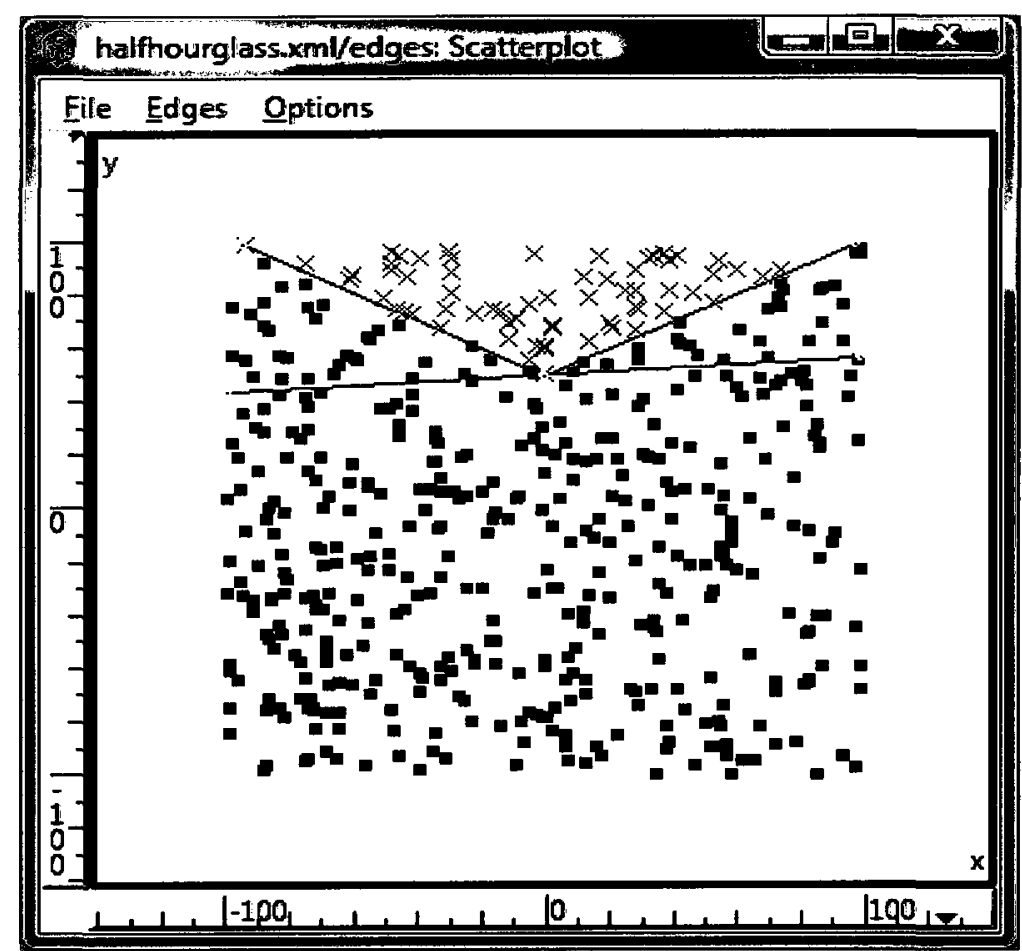

Figure 23: The final tree created by a pure cut hyperplane placement method
This hyperplane

isolates all the class 1

points on one side of the

hyperplane, and inspection

shows that perfect

classification of the data

could require as little as

two more hyperplanes.

After two more pure cuts, the final classification

appears as seen in Figure

23.

Pure cuts have performed better than the greedy hyperplane placement technique in this example; there are no misclassified points. Note that in the end, the initial hyperplane was rendered redundant. The final two hyperplanes are what truly separate out the class 1 points from the class 0 points. 


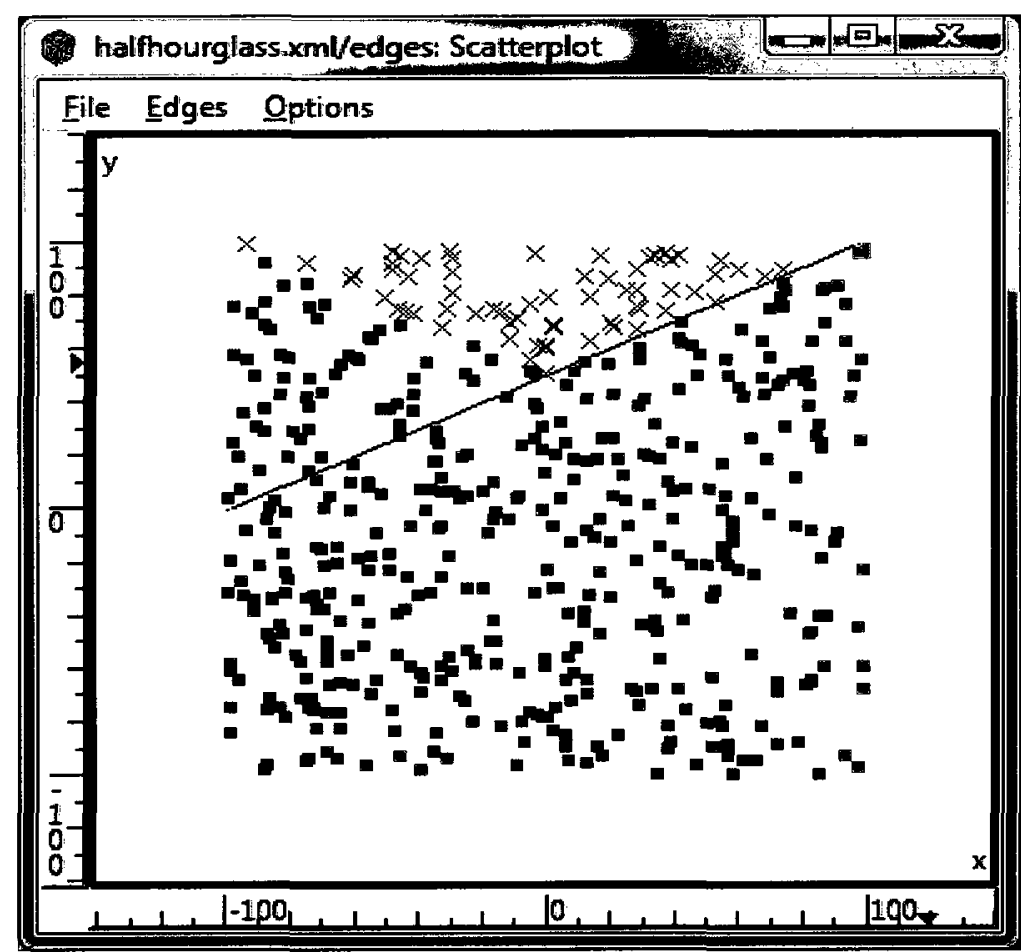

Figure 24: The first hyperplane, placed using a Edge Following hyperplane placement method
The idea behind

Edge Following cuts is to

place hyperplanes in

regions where two different

groups of data points meet.

Note that the class 1 points

form a triangle shaped

cluster in the graph. Two

edges of this triangle would

represent two regions

where different groups of data points meet. By placing hyperplanes along those two edges of the triangle, the data could be perfectly classified. The first of the two hyperplanes would appear as shown in Figure 24.

The placement of a second hyperplane along the other edge of the triangle correctly classifies all the data points, and more importantly, captures the true nature of the dataset. 


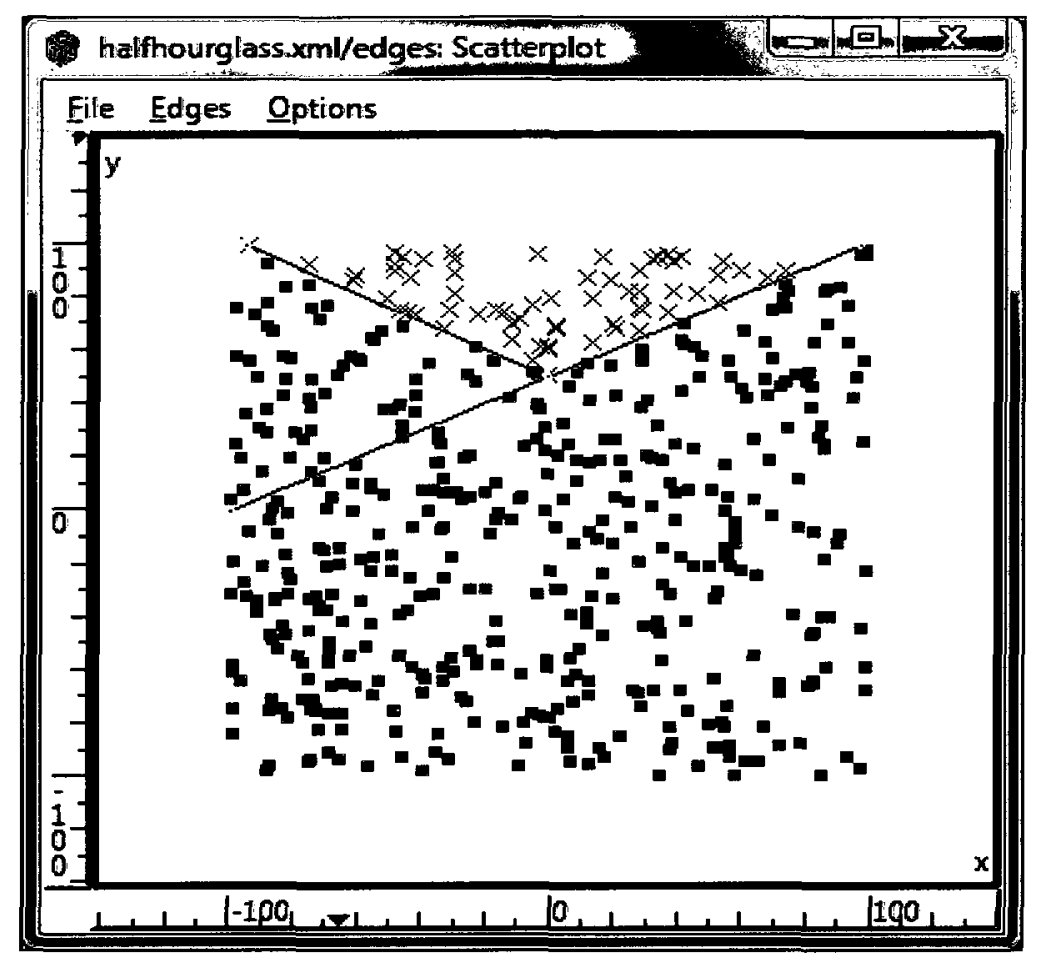

The final set of

hyperplanes is shown in

Figure 25.

The question is how

to elicit this sort of

decision from the

hyperplane placement

algorithm.

Figure 25: The final tree, created using an EF hyperplane placement method

\subsubsection{Edge Following (EF) Class Isolation Pure Cut Hyperplane Placement}

\section{Method}

The EF Class Isolation Pure Cut hyperplane placement method is best understood by an example:

Consider again the halfhourglass dataset. Since the goal is to place a hyperplane in a region where two different groups of data points meet, the first step is to identify an appropriate region. This is done by generating the $K N N$ subset by examining the $\mathrm{K}$ nearest neighbours of each data point in the data set. Any data point with a neighbour of the opposite class type is included in the KNN subset. For the halfhourglass dataset, the $\mathrm{KNN}$ subset for $\mathrm{k}=20$ appears as shown in Figure 26. 


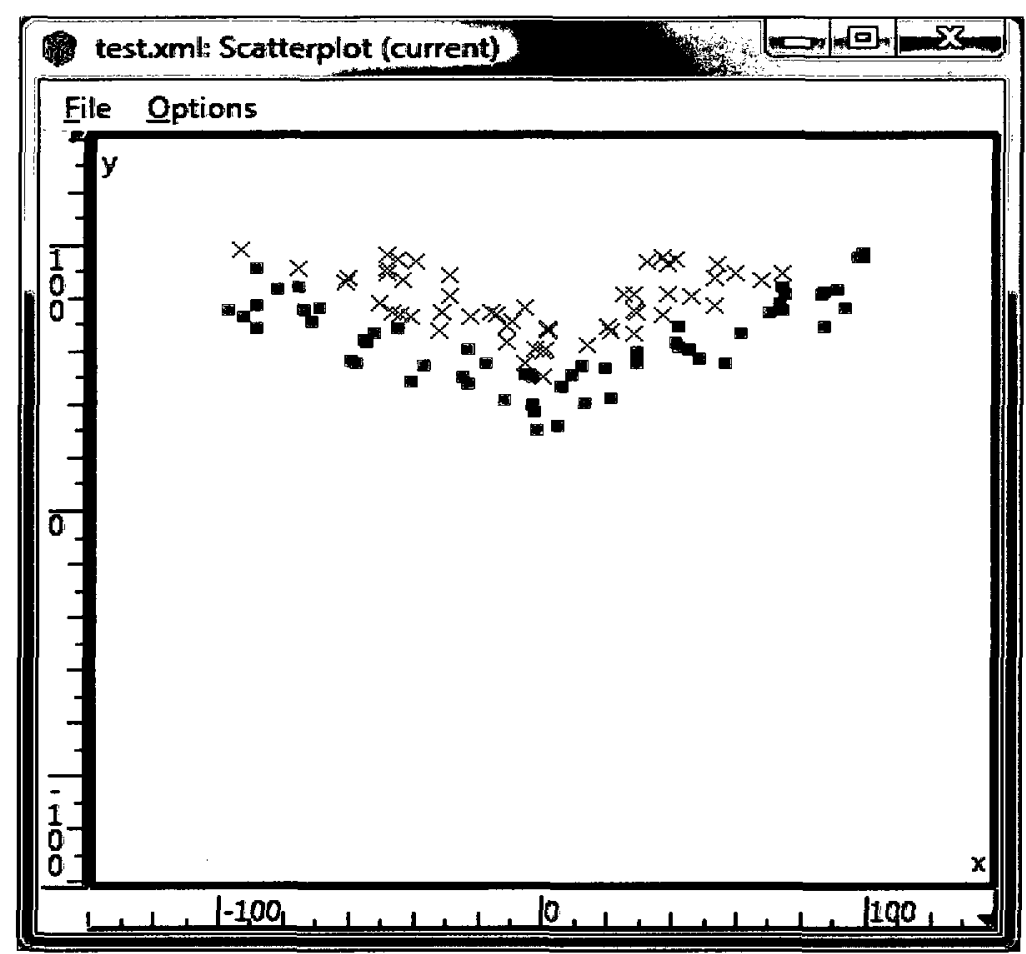

This process

eliminates large numbers

of data points that are in

homogenous regions of the

dataset. The points

remaining will be from

regions where points of

different class types are

found in close proximity,

Figure 26: The KNN subset of the halfhourglass dataset for $k=20$

and it is in these regions

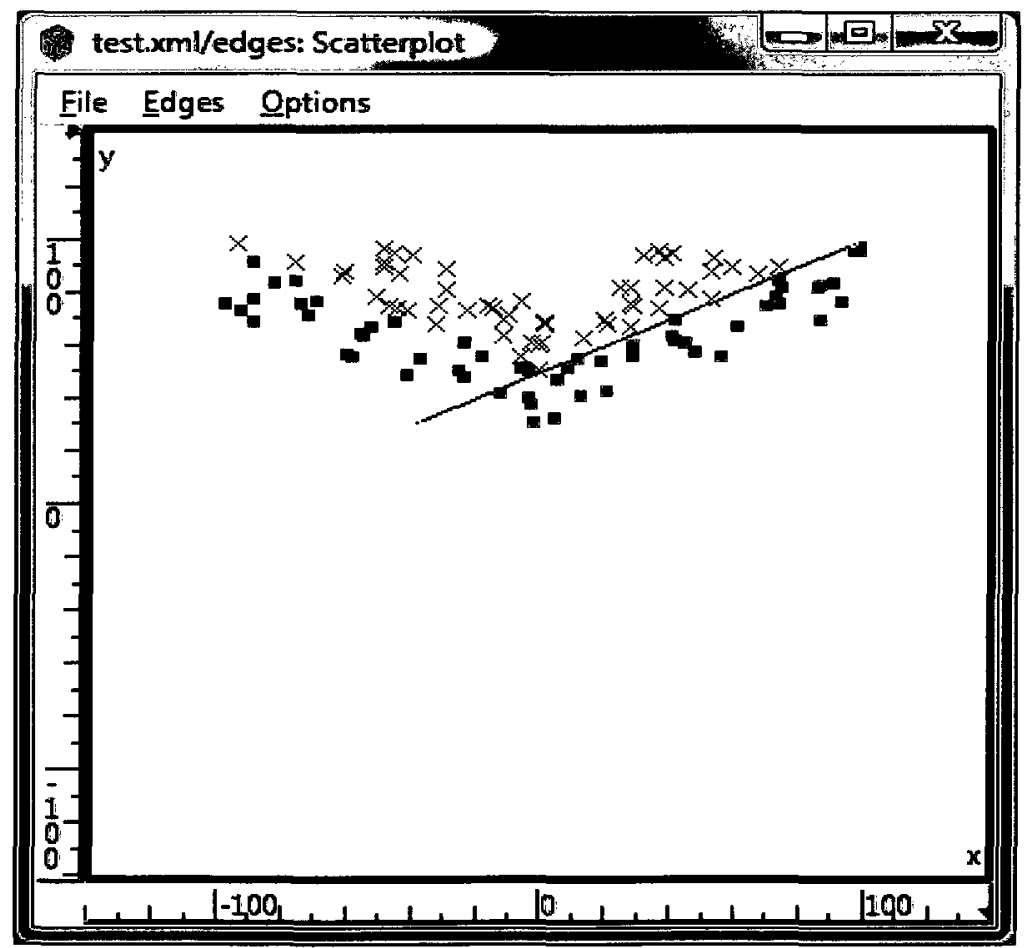

that separating surfaces are to be placed.

The next step in the process is to find the best pure cut. This is shown in Figure 27. Note how this cut slices right along the intersection of the two different classes. This cut becomes the first branch in

Figure 27: A pure cut placed in the KNN subset. the tree, shown in Figure 28. 


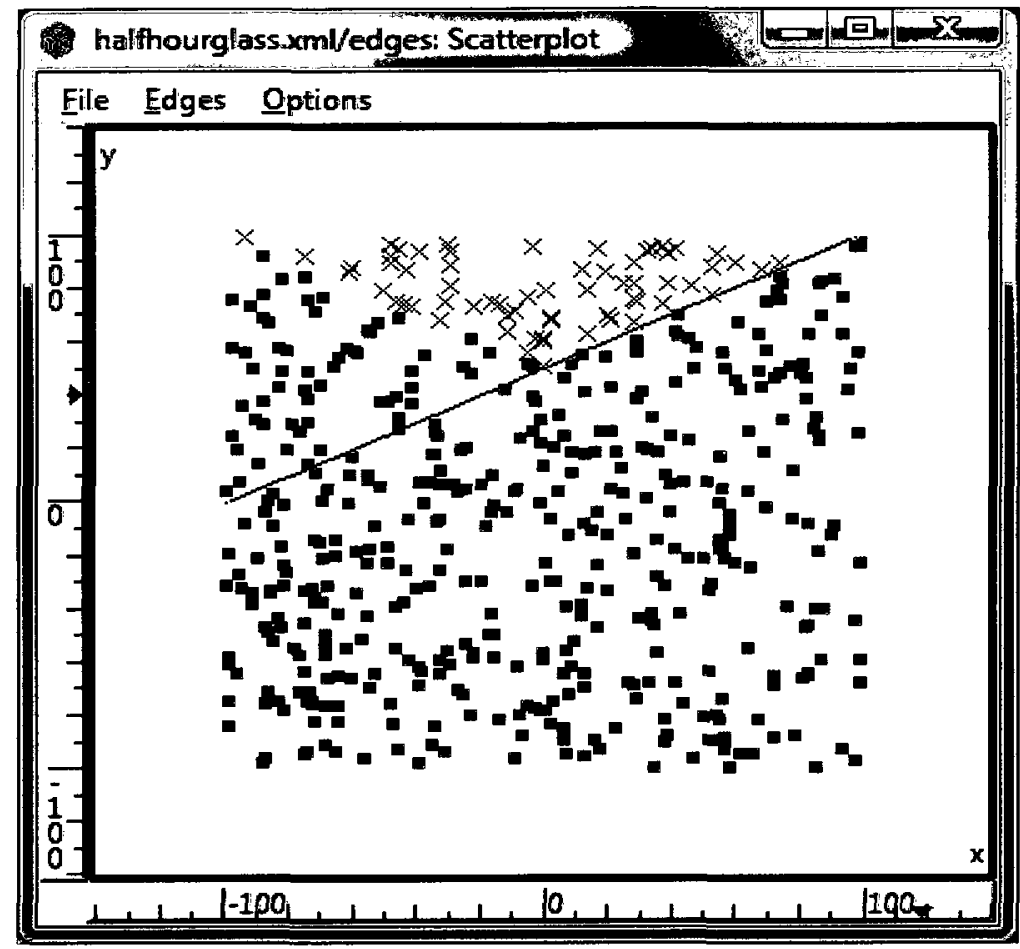

Repeating this

process results in a second

hyperplane being placed as

shown in Figure 29. The

EF Class Isolation Pure Cut

hyperplane placement

method has correctly

classified all the data, and

Figure 28: The same pure cut from the KNN subset, placed on the original dataset.

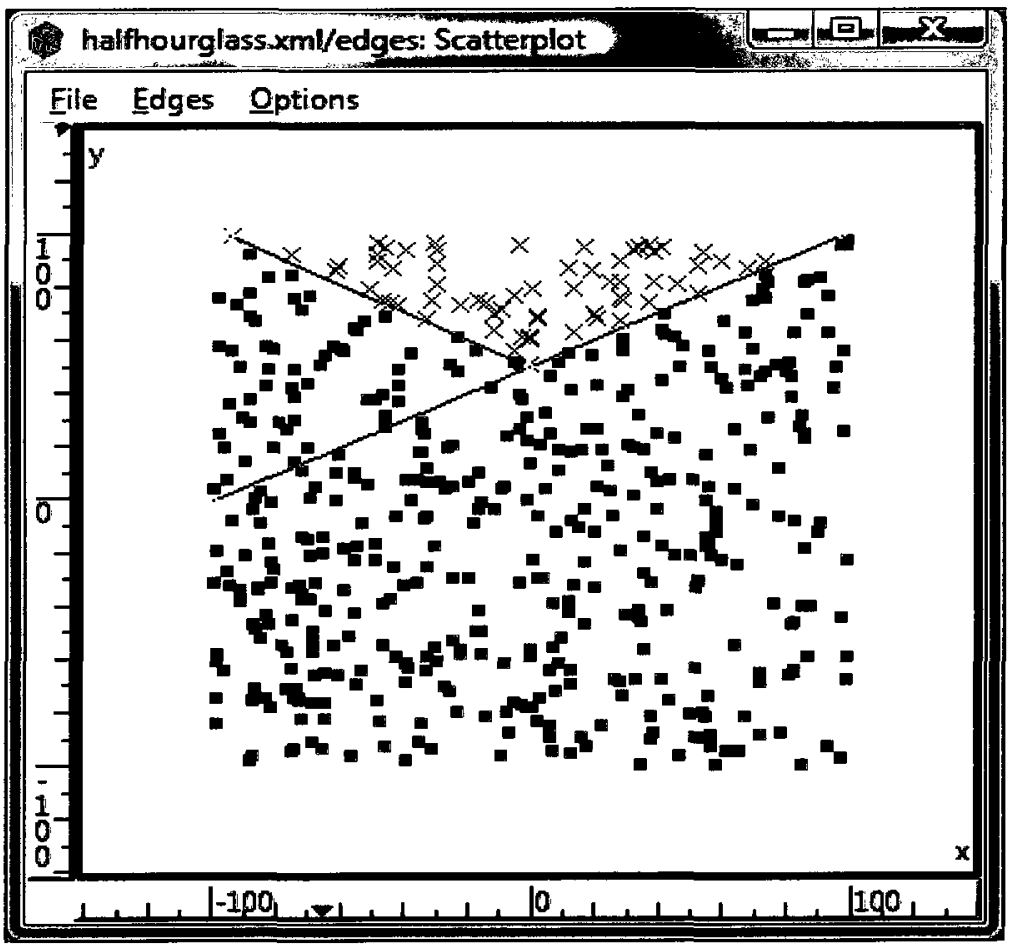

has done so with fewer

hyperplanes than the pure

cut hyperplane placement

method.

Pseudo code for the

EF Class Isolation Pure Cut

Hyperplane Placement

algorithm is shown in

Algorithm 3.

Figure 29: The final tree, created by the placement of two EF Class Isolation Pure Cut hyperplanes. 
INPUT: A dataset consisting of data points from two distinct classes: A and $B$

1) For each data point in the data set

a. Examine the $\mathrm{K}$ nearest neighbours

b. If any of those neighbours are of the opposite class, add this data point to the KNN subset.

2) Place a pure cut on the KNN subset.

OUTPUT: A pure cut hyperplane placed along an edge of intersection between classes A and B.

Algorithm 3: EF Class Isolation Pure Cut Hyperplane Placement

\subsubsection{Edge Following (EF) Class Isolation Greedy Cut Hyperplane Placement Method}

In most cases the region where two different groups of data points meet will contain a certain amount of overlap. The EF Class Isolation Greedy Cut hyperplane placement method attempts to place a hyperplane in this region of overlap, balancing the number of correctly and incorrectly classified data points according to a specified metric.

The EF Class Isolation Greedy Cut method uses the same first step that the EF Class Isolation Pure Cut method does. The mixed zone within which the hyperplane is to be placed still needs to be found. This mixed zone is found by taking the KNN subset of the original dataset. See Figure 26 in Section 5.1.2.1 for a diagram of the mixed zone for the halfhourglass dataset.

In the EF Class Isolation Pure Cut method, after the KNN subset is found, a pure cut hyperplane is then created for that subset. 


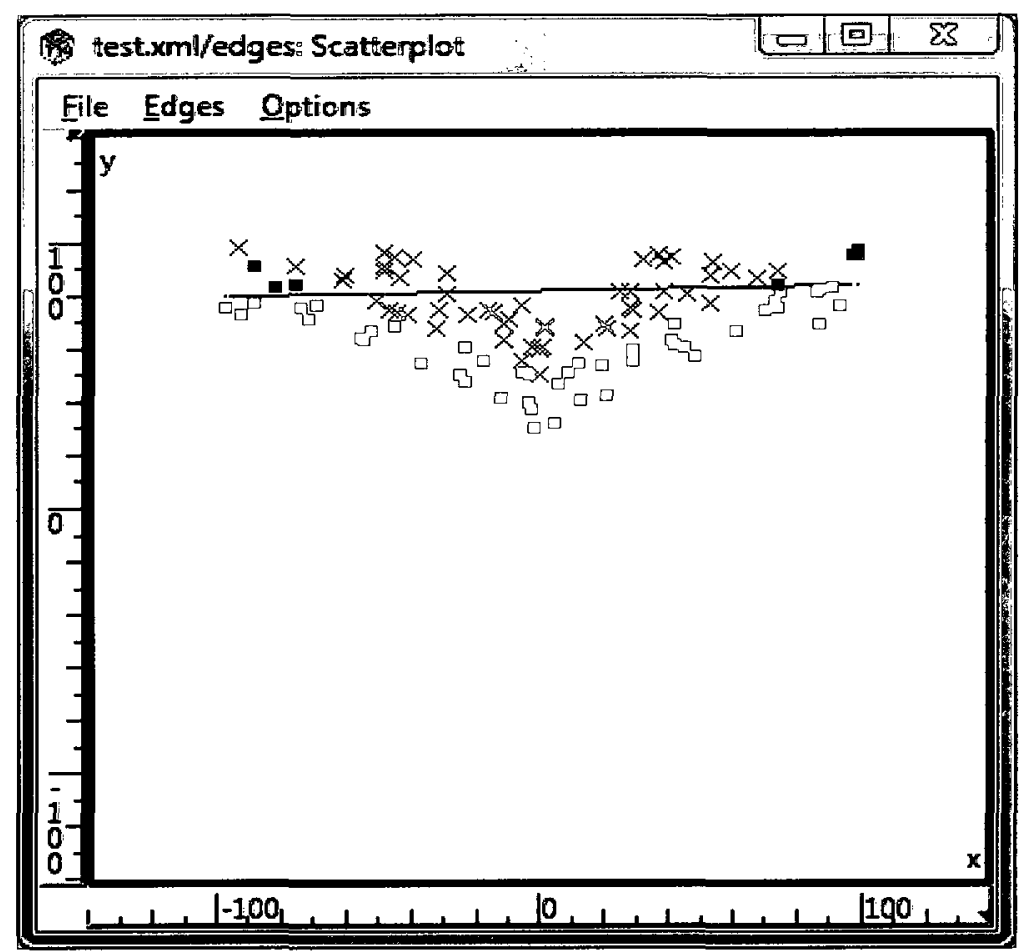

If, after creating

the KNN subset, a greedy

hyperplane placement

method is used instead, the

hyperplane seen in Figure

30 results. The greedy line

placement algorithm seeks

only to maximize for a

given metric, and thus

Figure 30: A greedy cut placed in the KNN subset doesn't work

arrives at a most unsatisfactory choice of hyperplane, such as the one seen in Figure 30.

In order to place a greedy line, some further preprocessing of this data is necessary. The goal here is to further reduce the data to a subset that contains one linear, or almost linear, region of separation. This further reduced dataset will be referred to as the linear subset.

The EF Class Isolation Pure Cut method offers a reasonable heuristic to obtain such a linear subset. Recall that the EF Class Isolation Pure Cut method will take the KNN subset and find the best pure cut that can be taken. Such a pure cut will tend to fall along the longest edge of intersection between two groups of data points of different class type, given a relatively uniform density of the data points (see Figure 27 in Section

\subsubsection{1 for a diagram).}

Now, instead of being kept, this cut is simply used to further reduce the 


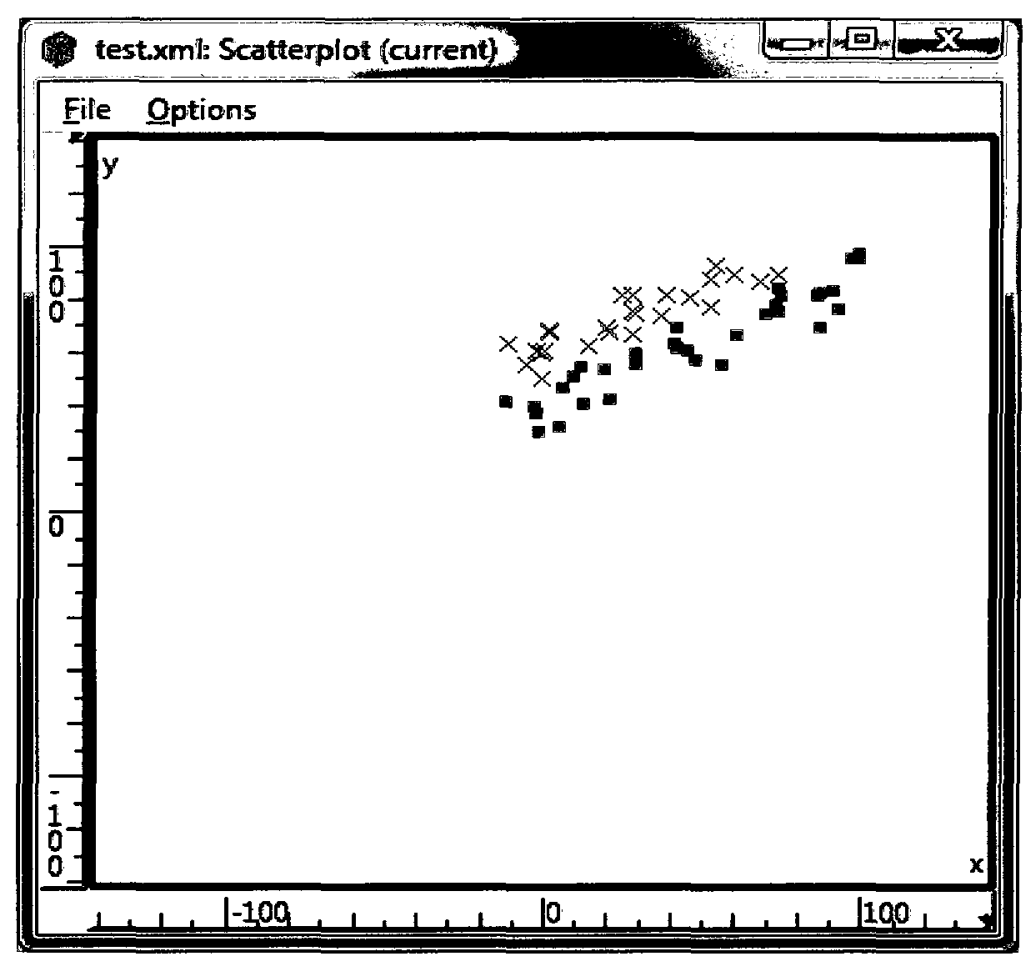

Figure 31: The linear subset

dataset. The linear subset includes all of the class 0 data points that the pure cut has just segregated AND all the type 1 points which were identified as nearest neighbours of those class 0 data points. All other points are discarded. The

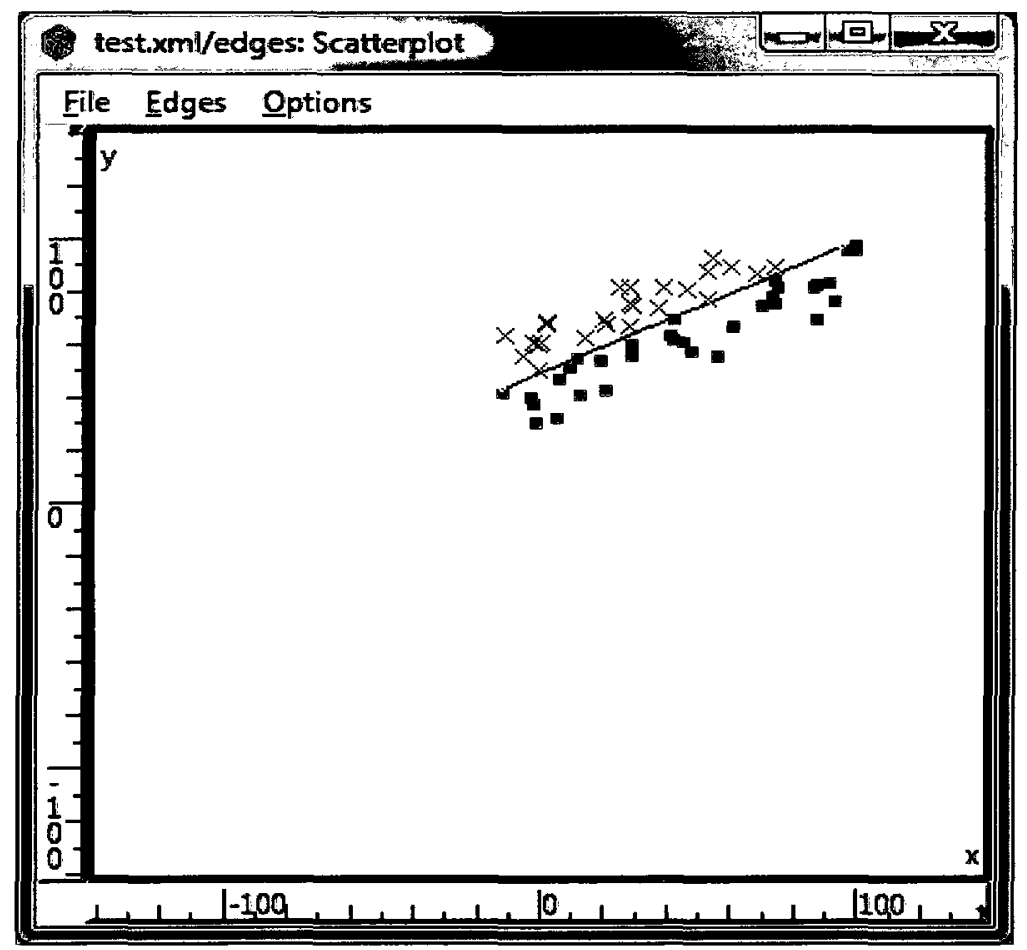

linear subset now consists of the data points shown in Figure 31 .

Now when a greedy hyperplane placement algorithm is used to separate these data points, the hyperplane shown in Figure 32 is produced.

Figure 32: A greedy cut placed in the linear subset 


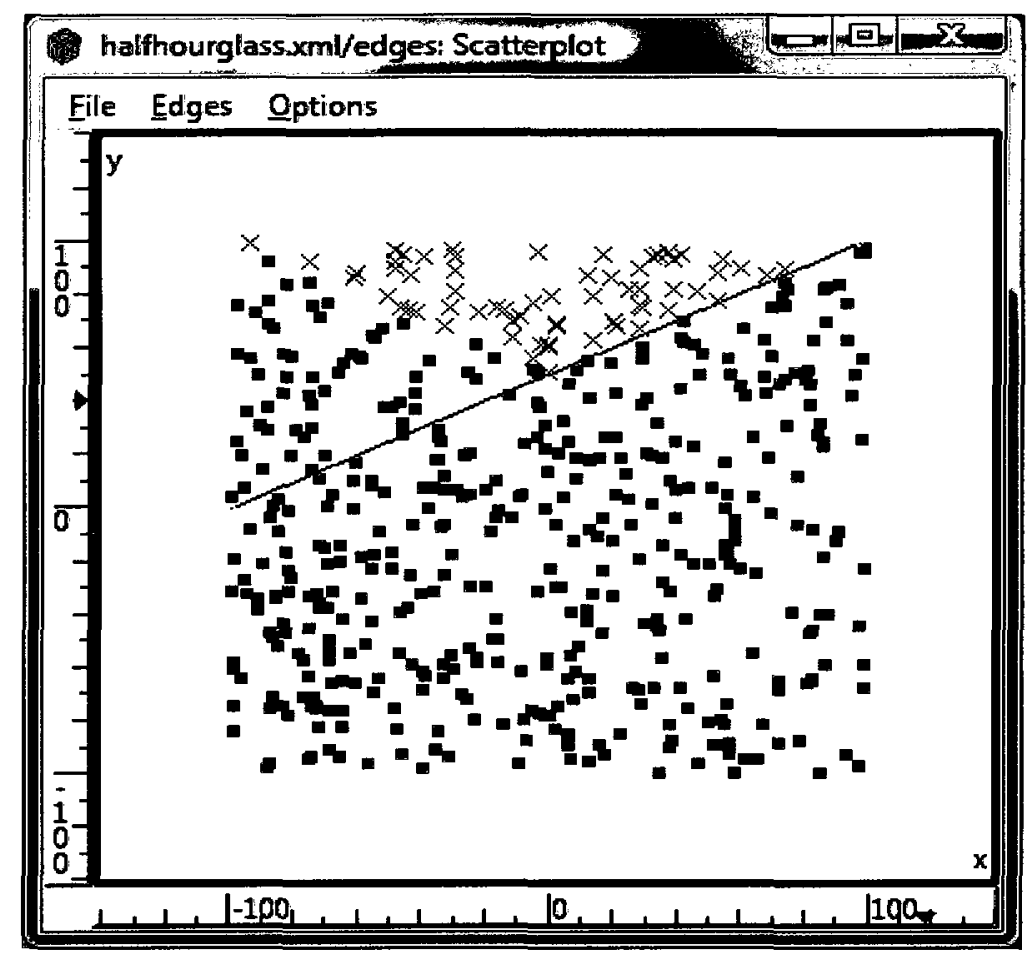

Figure 33: An EF Class Isolation Greedy Cut on the original dataset.

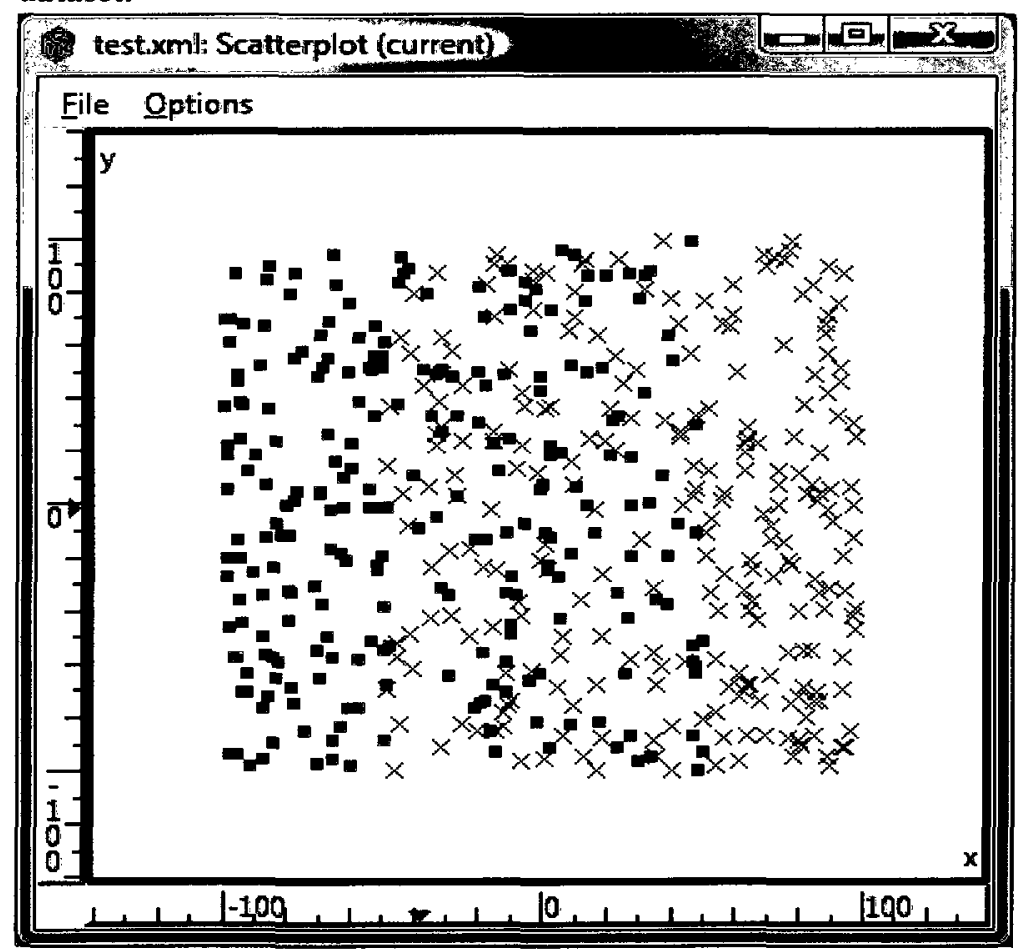

Figure 34: A sample dataset with a large mixed zone.
This hyperplane,

placed on the original

dataset appears as seen in

Figure 33.

\section{In order to}

accommodate datasets

where the zone of

intersection between class

0 and class 1 points is

significantly larger, the

algorithm described above

is organized into an

iterative process.

\section{Consider the}

dataset shown in Figure 
Applying the non-iterative approach, the diagram on the left hand side of Figure 35 shows the initial pure cut, placed on the KNN subset of the dataset shown in Figure

34. The diagram on the right side of Figure 35 shows the linear subset.

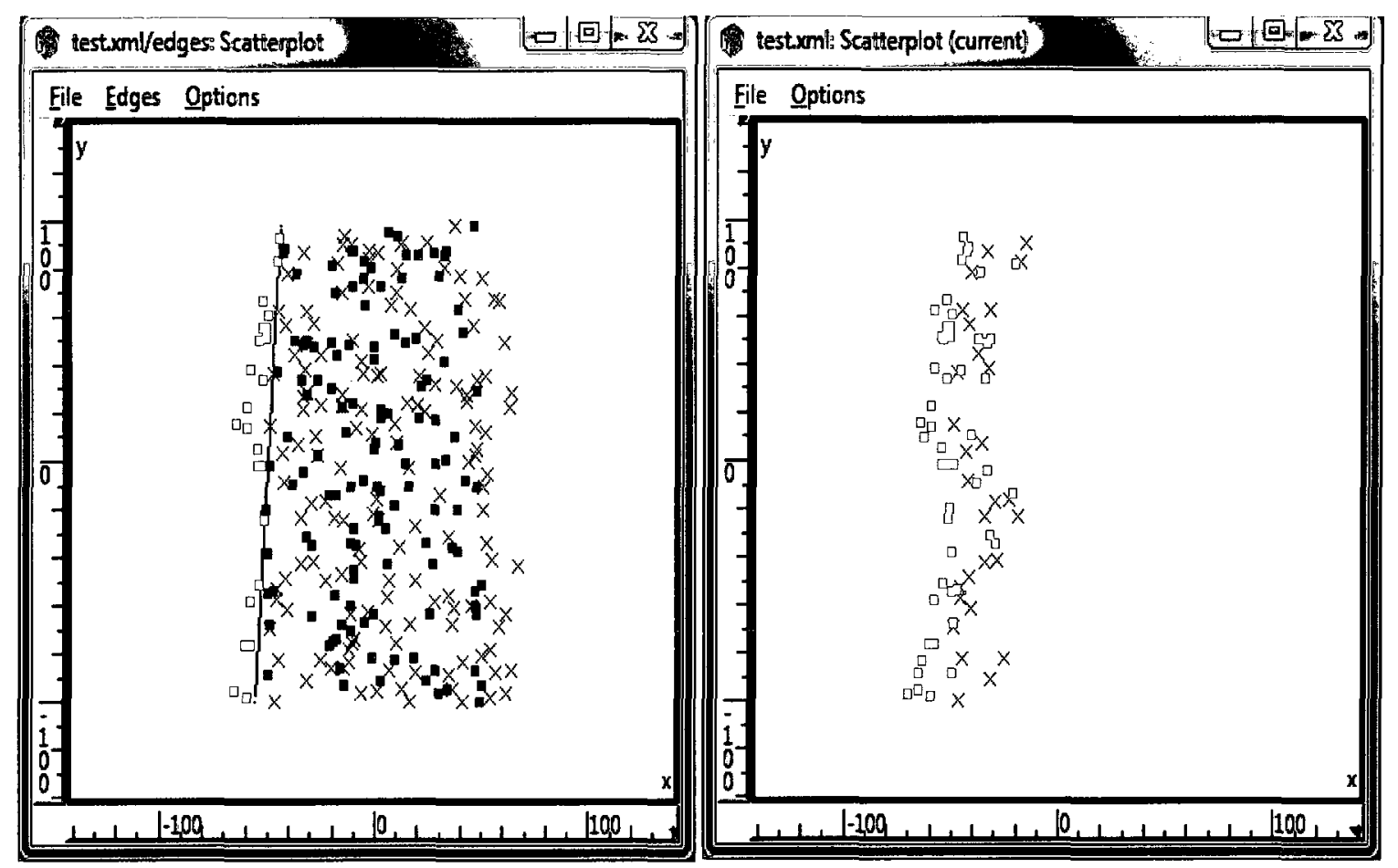

Figure 35: A pure cut is taken on the KNN sub-dataset. The sub-dataset is then further reduced to the linear subset.

The diagram on the left of Figure 36 shows a greedy hyperplane placed in the linear subset, but when this hyperplane is viewed against the original dataset, it is fairly clear that this is not an ideally placed hyperplane. 


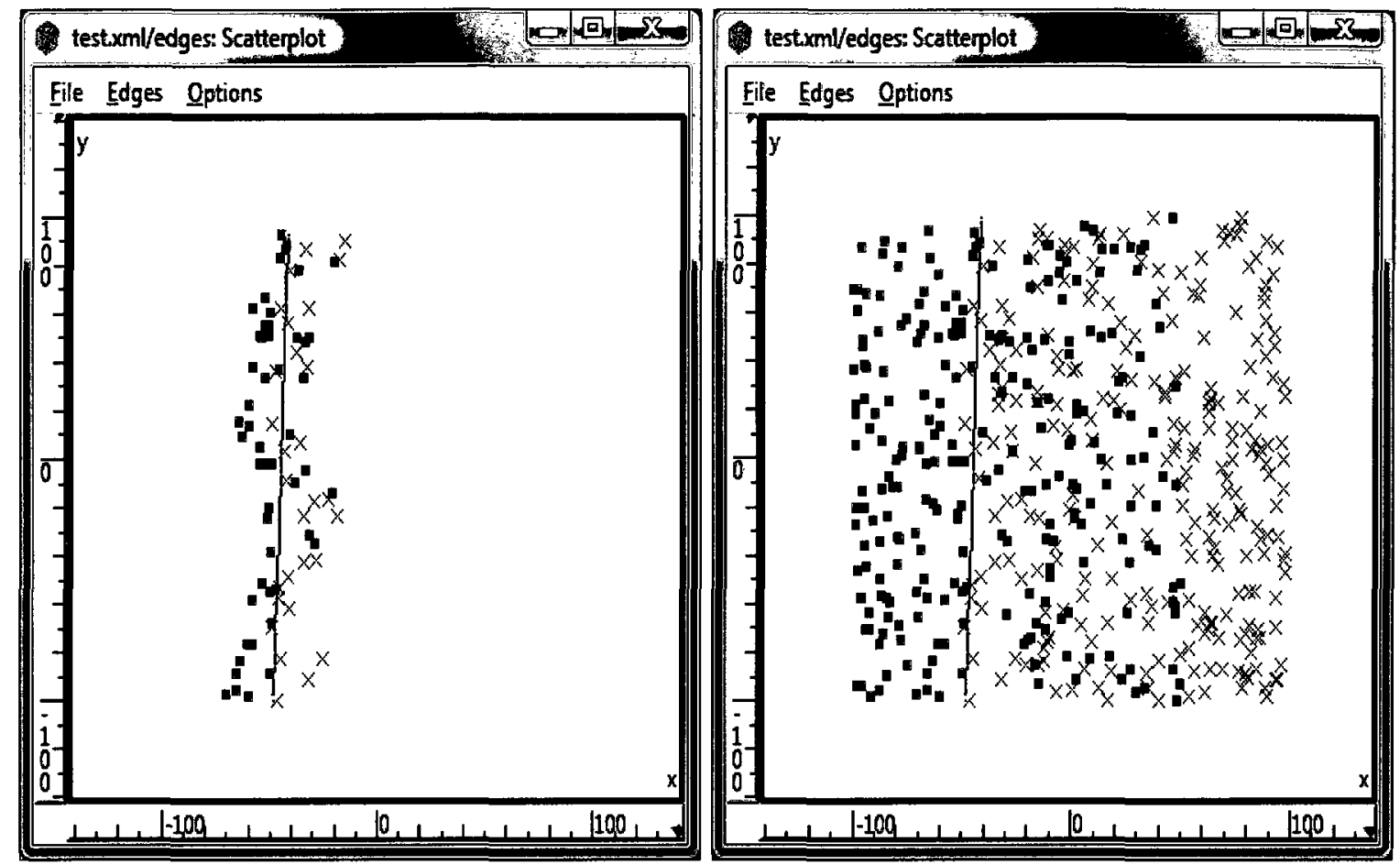

Figure 36: A greedy cut is placed in the linear subset, but this greedy cut when viewed against the full data set doesn't make much sense.

The problem with the non iterative approach is that it fails to include all points in the mixed zone for consideration and thus results in a greedy line placement that is clearly not correct. A close examination of Figure 35 highlights the problem. The graph on the left (the KNN subset) shows that the mixed zone extends from roughly $x=-60$ to $\mathrm{x}=60$. The graph on the right hand side of Figure 35 shows the linear subset, but the mixed zone data points in this subset extend from roughly $\mathrm{x}=-60$ to $\mathrm{x}=-20$. Thus when the greedy cut is taken, only a portion of the mixed zone data points are being used, resulting in a hyperplane that is improperly placed.

To avoid this problem, the EF Class Isolation Greedy Cut hyperplane placement algorithm is organized into an iterative process, outlined in Algorithm 4. 
INPUT: A dataset consisting of data points from two distinct classes: A and $B$

1) For each data point in the data set:

a. Examine the $\mathrm{K}$ nearest neighbours

b. If any of those neighbours are of the opposite class, add this data point to the KNN subset.

2) Place a pure cut on the KNN subset. Label the points in the pure zone as being of class $\mathrm{C}$. All points not in class $\mathrm{C}$ are labeled as being of class $\mathrm{D}$.

3) Create a linear subset composed of all points in class $C$, and all class D points that are among the $k$ nearest neighbours of class $C$ points

4) While the linear subset contains no class $D$ data points that are in a pure zone:

a. Find the nearest Class $D$ neighbours (in the original dataset) of the class $D$ data points in the linear subset using the KNN method.

b. Add them to the linear subset.

5) The linear subset now contains all the class $D$ data points needed. Complete the linear subset by including all class $C$ data points that are neighbours of those class $D$ data points.

6) Apply a greedy hyperplane placement algorithm to place a hyperplane through the linear subset.

OUTPUT: A greedy hyperplane placed along an edge of intersection between classes A and B.

Algorithm 4: EF Class Isolation Greedy Cut Hyperplane Placement

Applying this algorithm to the sample dataset under consideration, Step 4 of

Algorithm 4 grows the mixed zone as shown in Figure 37 to Figure 39. 


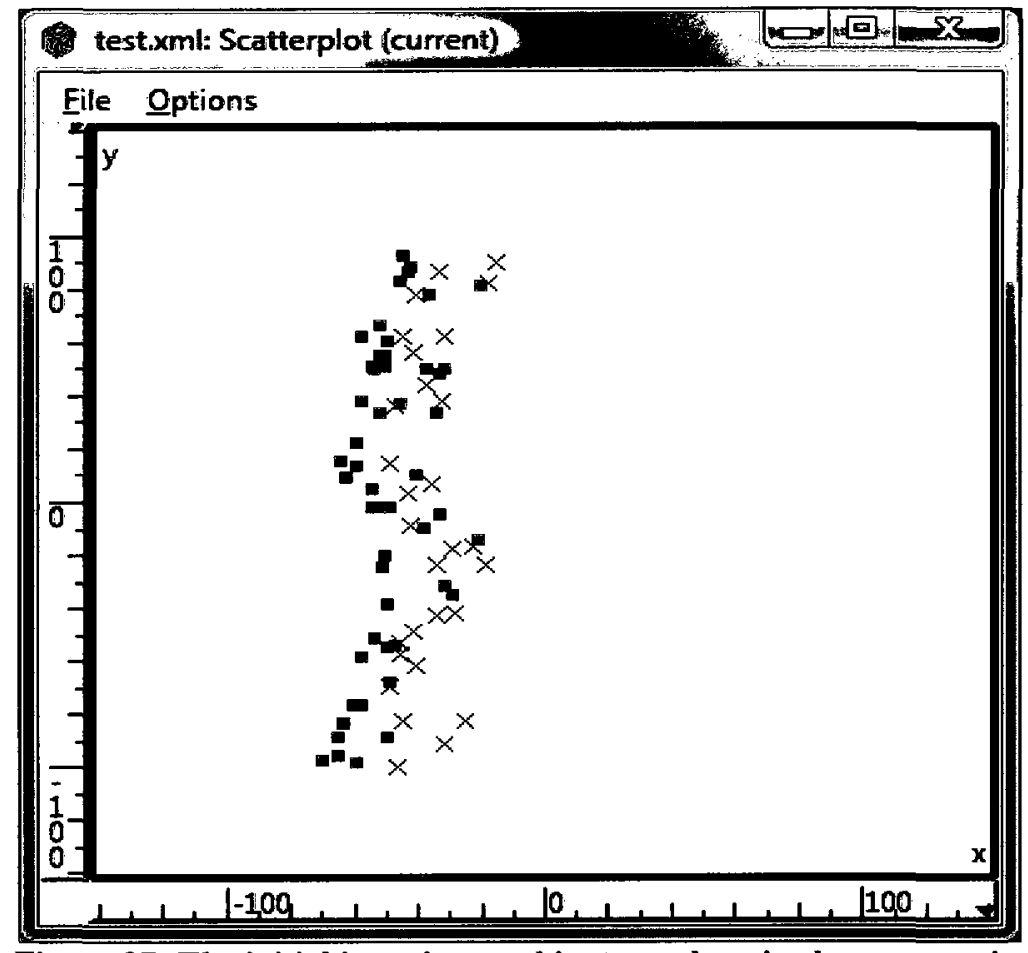

Figure 37: The initial iteration, at this stage, the mixed zone contained in the linear subset extends only to about $x=-20$
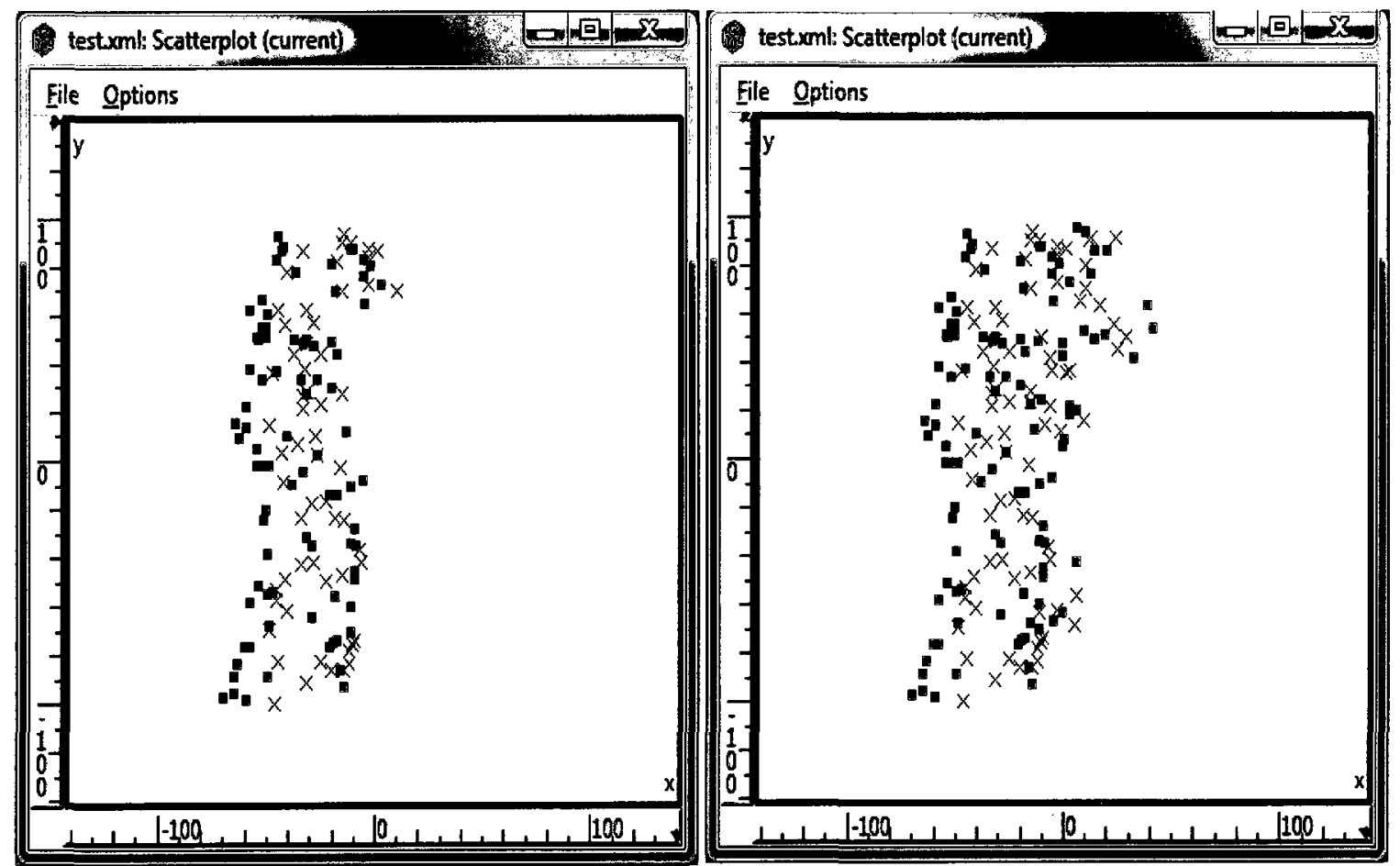

Figure 38: After 2 more iterations, a larger section of mixed zone points is now incorporated into the linear subset. 


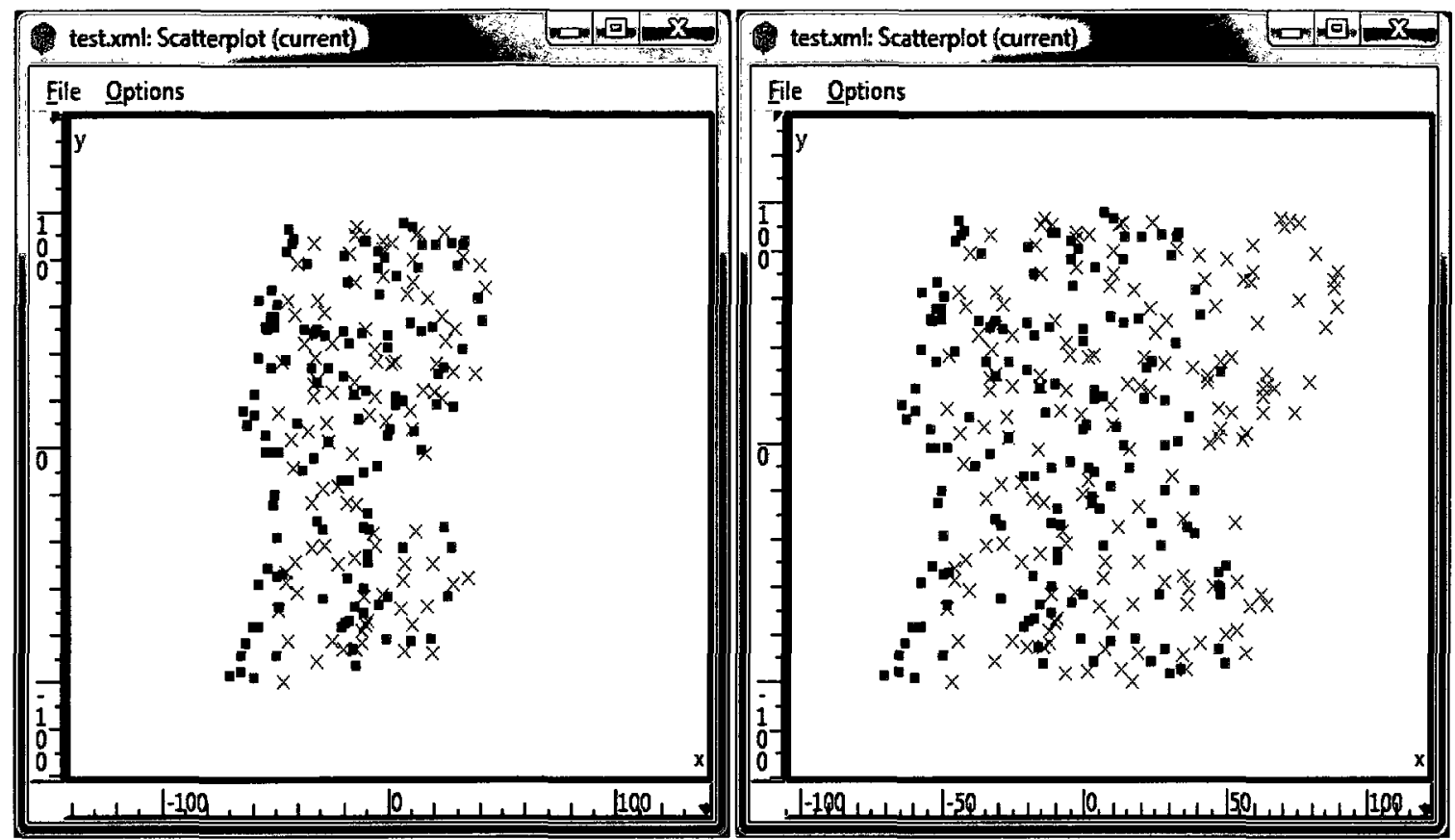

Figure 39: After a couple more iterations, the complete mixed zone is now incorporated into the linear subset.

Now that the iterative process in Step 3 of Algorithm 4 has retrieved all points in the mixed zone, an appropriate greedy cut can now be made as shown in Figure 40.
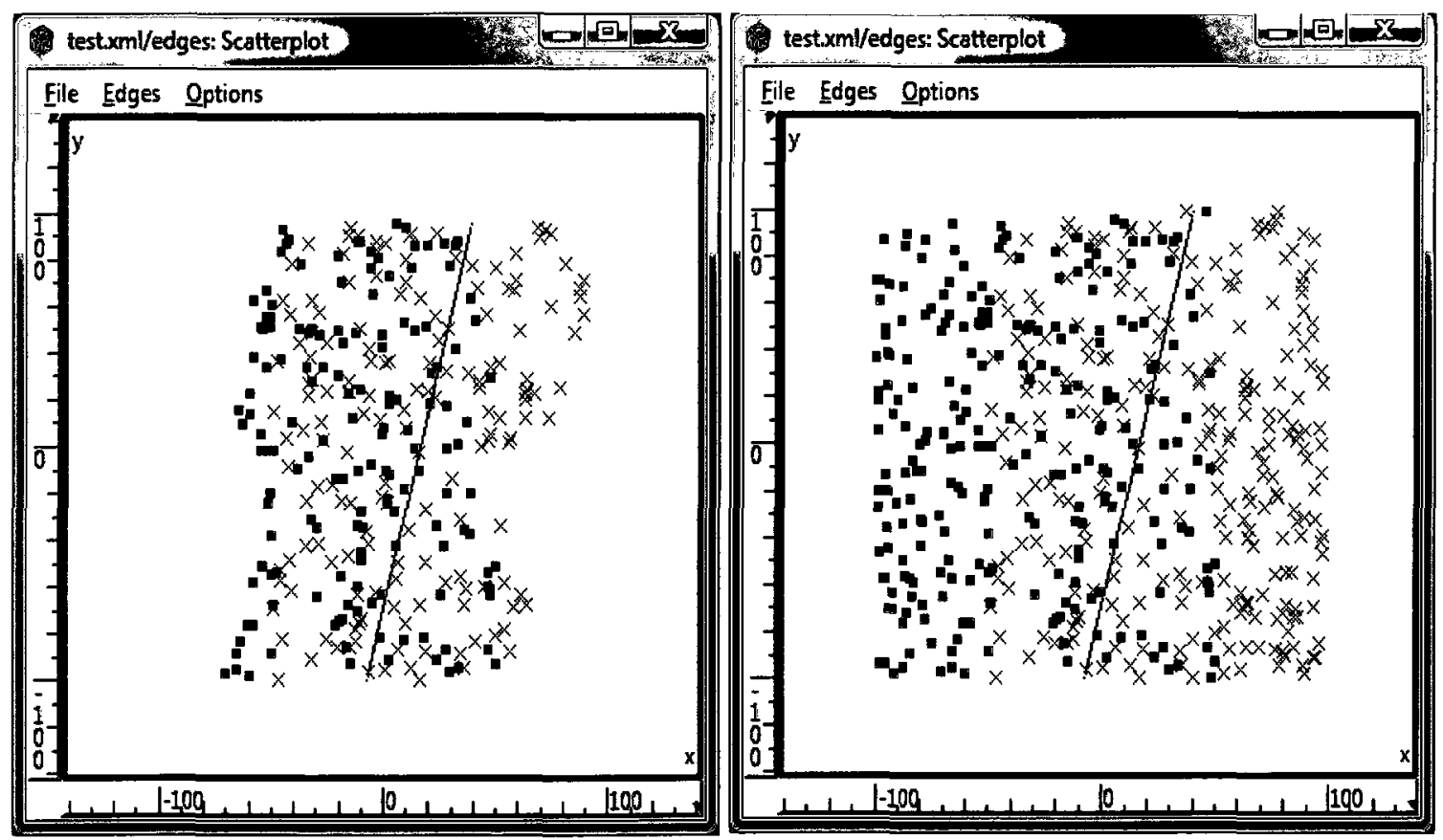

Figure 40: The final hyperplane, seen against the linear subset and against the original dataset 


\subsubsection{Edge Following (EF) Mixed Isolation Pure Cut Hyperplane Placement Method}

The EF Mixed Isolation Pure Cut method functions the same way as the EF Class Isolation Pure Cut method does, except that it is applied to a transformed dataset. The transformed dataset assigns data points the class of $P$ for those data points that are in pure regions and $M$ for those data points that are found to be in mixed regions. Determining if a data point is found in a mixed region is done by examining the $K$ nearest neighbours of that data point. If any of those nearest neighbours are of the opposite class type then the data point in question is in a mixed zone.

Pseudo code for the EF Mixed Isolation Pure Cut Hyperplane Placement algorithm is shown in Algorithm 5. Note that $K_{m z t}$ refers to the number of nearest neighbours examined to determine if a data point is considered to be in a mixed zone in the transformed data set, $K_{\mathrm{mz}}$ refers to the number of nearest neighbours examined to determine if a data point is considered to be in a mixed zone when creating the $\mathrm{KNN}$ subset.

The use of $\mathrm{K}_{\mathrm{mzt}}$ and $\mathrm{K}_{\mathrm{mz}}$ allows for flexibility. For $\mathrm{K}_{\mathrm{mz}}$, a value of less than ten seems to result in such small KNN subsets that the EF Hyperplane Placement Methods start producing poor hyperplanes. An upper bound is difficult to determine, but generally speaking, if $\mathrm{K}_{\mathrm{mz}}$ is set to be more than $30 \%$ of the total data points, the EF methods start exhibiting behavior similar to ordinary greedy hyperplane placement methods. The value of $K_{m z t}$ is a matter of personal preference, and comes down to a trade off: A large value of $\mathrm{K}_{\mathrm{mzt}}$ results in a larger number of seemingly pure zone data points being included in 
the Mixed Zone portion of the dataset, while a low value may result in small patches of data points in a seemingly mixed zone section of the dataset being labeled as being pure.

INPUT: A dataset consisting of data points from two distinct classes: $A$ and $B$

1) For each data point in the data set

a. Examine the $\mathrm{K}_{\mathrm{mzt}}$ nearest neighbours

b. If any of those neighbours are of the opposite class, add this data point to the Transformed dataset as a class $\mathrm{M}$ point. Otherwise add it to the Transformed data set as a class $P$ point

2) For each data point in the transformed data set

a. Examine the $\mathrm{K}_{\mathrm{mz}}$ nearest neighbours

b. If any of those neighbours are of the opposite class, add this data point to the KNN subset.

3) Place a pure cut on the KNN subset.

OUTPUT: A pure cut hyperplane placed along an edge of intersection between a homogenous group of data points and a heterogeneous group of data points.

Algorithm 5: EF Mixed Isolation Pure Cut Hyperplane Placement

\subsubsection{Edge Following (EF) Mixed Isolation Greedy Cut Hyperplane Placement}

\section{Method}

The EF Mixed Isolation Greedy Cut method functions the same way as the EF

Class Isolation Greedy Cut method does, except that it is applied to a transformed dataset.

The transformed dataset assigns data points the class of $P$ for those data points that are in pure regions and $M$ for those data points that are found to be in mixed regions.

Determining if a data point is found in a mixed region is done by examining the $\mathrm{K}$ nearest neighbours of that data point. If any of those nearest neighbours are of the opposite class type then the data point in question is in a mixed zone.

Pseudo code for this algorithm is shown in Algorithm 6. Note that $\mathrm{K}_{\mathrm{mzt}}$ will refer to the number of nearest neighbours examined to determine if a data point is considered to be in a mixed zone in the transformed data set, $\mathrm{K}_{\mathrm{mz}}$ will refer to the number of nearest 
neighbours examined to determine if a data point is considered to be in a mixed zone when creating the KNN subset.

INPUT: A dataset consisting of data points from two distinct classes A and B:

1) For each data point in the data set:

a. Examine the $\mathrm{K}_{\mathrm{mzt}}$ nearest neighbours

b. If any of those neighbours are of the opposite class, add this data point to the Transformed dataset as a class $M$ point. Otherwise add it to the transformed data set as a class $P$ point

2) For each data point in the transformed data set:

a. Examine the $\mathrm{K}_{\mathrm{mz}}$ nearest neighbours

b. If any of those neighbours are of the opposite class, add this data point to the KNN subset.

3) Place a pure cut on the KNN subset. Label the points in the pure zone as being of class C. All points not in class $\mathrm{C}$ are labeled as being of class $\mathrm{D}$.

4) Create a linear subset composed of all points in class $C$, and all class $D$ points that are among the $\mathrm{k}$ nearest neighbours of class $\mathrm{C}$ points

5) While the linear subset contains no class $D$ data points that are in a pure zone:

a. Find the nearest Class $D$ neighbours (in the original dataset) of the class $D$ data points in the linear subset using the KNN method.

b. Add them to the linear subset.

6) The linear subset now contains all the class $D$ data points needed. Complete the linear subset by including all class $C$ data points that are neighbours of those class $\mathrm{D}$ data points.

7) Apply a greedy hyperplane placement algorithm to place a hyperplane through the linear subset.

OUTPUT: A greedy hyperplane placed along an edge of intersection between a homogenous group of data points and a heterogeneous group of data points. 


\subsection{Policies}

\subsubsection{Policies Employing Greedy Hyperplane Placement Techniques}

There are several existing metrics that can be used to measure the performance of a hyperplane (see section 2.1.2). Using modifications to the basic LP based hyperplane placement algorithm, proposed by Chinneck (2009), LP based hyperplane placement methods that maximize for Gini Index, entropy, EROC, and a cost function have been developed and implemented.

These methods, along with the basic LP based hyperplane placement method which maximizes for accuracy, are then applied in policies in an effort to produce better performing decision trees. A policy is a set of rules that are used to determine what hyperplane placement technique to use at a given node. The goal of this research is to find a way of determining which greedy policy to use at a given node that will maximize the overall performance of the decision tree, rather than maximizing the performance of the given node.

\subsubsection{Policies Employing Non-Greedy Hyperplane Placement Techniques}

\subsubsection{Fixed Order Policies}

Fixed order policies are policies in which hyperplane placement methods are applied in a rigid order. If a policy is set to apply hyperplane placement method 1 and method 2 (in that order), then at each node, the policy attempts to place a hyperplane using method 1. If it is unable to create a hyperplane using method 1 , then it attempts to place a hyperplane using method 2. 
Different hyperplane placement methods may fail for different reasons. Edge Following methods will fail if they generate a KNN subset or a linear subset consisting of too few data points. Any hyperplane placement method will fail if an unresolvable degeneracy is encountered. A greedy hyperplane placement method will fail if the hyperplane produced results in no improvement to the overall tree.

There are 15 fixed order policies proposed and tested in this thesis. The hyperplane placement methods used, and the order in which they are attempted is specified in Table 4. Note that in Table 4 "Greedy", indicates the use of Chinneck's (2009) greedy hyperplane placement method, where the metric being maximized is specified by the user as a parameter.

Table 4: Fixed Order Policies

\begin{tabular}{|l|l|l|l|}
\hline $\begin{array}{l}\text { Policy } \\
\text { Number }\end{array}$ & Method 1 & Method 2 & Method 3 \\
\hline 1 & Pure Cut & Greedy & none \\
\hline 2 & Mixed Zone Cut & Greedy & none \\
\hline 3 & EF Class Isolation Pure Cut & Greedy & none \\
\hline 4 & EF Class Isolation Greedy & Greedy & none \\
\hline 5 & EF Mixed Isolation Pure Cut & Greedy & none \\
\hline 6 & EF Mixed Isolation Greedy & Greedy & none \\
\hline 7 & Pure Cut & Mixed Zone Cut & Greedy \\
\hline 8 & Pure Cut & EF Mixed Isolation Cut & Greedy \\
\hline 9 & Pure Cut & EF Mixed Isolation Greedy & Greedy \\
\hline 10 & EF Class Isolation Pure Cut & Mixed Zone Cut & Greedy \\
\hline 11 & EF Class Isolation Pure Cut & EF Mixed Isolation Pure Cut & Greedy \\
\hline 12 & EF Class Isolation Pure Cut & EF Mixed Isolation Greedy & Greedy \\
\hline 13 & EF Class Isolation Greedy & Mixed Zone Cut & Greedy \\
\hline 14 & EF Class Isolation Greedy & EF Mixed Isolation Pure Cut & Greedy \\
\hline 15 & EF Class Isolation Greedy & EF Mixed Isolation Greedy & Greedy \\
\hline
\end{tabular}

There are a vast number of fixed order policies that could be created using the various hyperplane placement methods seen in Table 4. Testing all possible 
combinations is infeasible. The 15 methods shown in Table 4 were chosen due to the following reasons:

- If a Class Isolation method is employed and fails, the vast majority of the time other Class Isolation methods also fail. The same applies for Mixed Isolation methods. Thus if a policy first applies a Class Isolation method, all subsequent methods should apply either mixed Isolation methods, Pure Cuts, or a greedy method.

- The use of a greedy method as the first method applied tends to produce inferior results. Greedy methods rarely fail until late in the tree creation process, and in many cases, non-greedy methods are then unable to correct the errors made by their greedy counterparts.

\subsubsection{Partially Dynamic Policies}

Partially Dynamic policies introduce a certain amount of selection to the process of choosing which hyperplane placement method will be used.

In all Edge Following methods, the region where two different groups of data points meet is found by generating the $\mathrm{KNN}$ subset. A pure cut hyperplane is then placed on the KNN subset. This hyperplane will usually fall along the longest edge of separation between two groups of data points. Generally speaking, the more data points found to be separated out by the pure cut (this number will be referred to as the $K N N$ subset metric), the longer the edge of separation between two groups of data points. The partially dynamic policies all rely on this as a means of determining whether or not to employ an EF Mixed Isolation method or an EF Class Isolation method. The method whose $\mathrm{KNN}$ subset metric is larger is the method chosen. 
It should be noted that all partially dynamic policies choose between an EF Class Isolation method and an EF Mixed Isolation Method. Creating a partially dynamic policy that chooses between the two EF Class Isolation Methods (or the two EF Mixed Isolation Methods) would be pointless: their KNN subsets would be identical, and thus their KNN subset metrics would be the same.

Table 5: Partially Dynamic Policies

\begin{tabular}{|l|l|l|}
\hline $\begin{array}{c}\text { Policy } \\
\text { Number }\end{array}$ & \multicolumn{1}{|c|}{ Choose Between } & \multicolumn{1}{|c|}{ Fall Back } \\
\hline 16 & $\begin{array}{l}\text { EF Class Isolation Pure Cut and } \\
\text { EF Mixed Isolation Greedy Cut }\end{array}$ & greedy \\
\hline 17 & $\begin{array}{l}\text { EF Class Isolation Greedy Cut and } \\
\text { EF Mixed Isolation Greedy Cut }\end{array}$ & greedy \\
\hline 18 & $\begin{array}{l}\text { EF Class Isolation Pure Cut and } \\
\text { EF Mixed Isolation Pure Cut }\end{array}$ & greedy \\
\hline 19 & $\begin{array}{l}\text { EF Class Isolation Greedy Cut and } \\
\text { EF Mixed Isolation Pure Cut }\end{array}$ & greedy \\
\hline
\end{tabular}

The pseudo code representing the generic approach employed by the dynamic policies is given in Algorithm 7.

INPUT: A dataset consisting of data points from two distinct classes: $A$ and $B$

1) Attempt to create two candidate hyperplanes, one for each of the Edge Following methods employed by the policy, and note the KNN subset metric for each method.

2) If no candidate hyperplanes are created:

a. Attempt to create a hyperplane using the Fallback method. If this fails, return a NULL hyperplane and exit.

3) If only one candidate hyperplane is created:

a. Return the one candidate hyperplane and identify the method used to create it.

4) If two candidate hyperplanes are created:

a. Return the candidate with the larger KNN subset metric and identify the method used to create it.

OUTPUT: A hyperplane, and the identity of the Edge Following method used to create it 


\subsubsection{Fully Dynamic Policies}

Fully Dynamic Policies can choose from any of the 4 Edge Following methods when it comes to deciding what hyperplane placement method to use at a given node. When making this determination, the first step is to determine whether or not to use an EF Mixed Isolation method or an EF Class Isolation method. As with Partially Dynamic Policies, the Edge Following method with a larger KNN subset metric is chosen.

The second step is to determine whether to use a greedy or a pure cut version of the Edge Following Method selected. This determination is made by examining the linear subset created by the greedy version. If the mixed zone of data points in the linear subset is large, a pure cut version of the Edge Following Method selected is used. If the mixed zone is small, then a greedy cut version of the Edge Following Method selected is used.

Recall that Algorithm 4 describes an iterative process used to place an EF Class Isolation Greedy Cut (the same iterative approach is employed in Algorithm 6 for EF Mixed Isolation Greedy Cuts). The iterative portion of these algorithms ensures that the linear subset contains the entire mixed zone between two different groups of data points. The more iterations it takes to include the entire mixed zone, the larger the mixed zone is. In policies 20,21 , and 22 , the number of iterations needed is used to determine whether or not to employ a greedy or a pure version of the Edge Following method selected in Step 1. The maximum number of iterations allowed before policy 20,21 , or 22 choose to use a pure cut version is 1,2 , and 3 respectively. Policy 23 uses a fixed percentage to determine whether or not to use a pure cut or greedy cut version of the Edge Following 
Method selected. If the mixed zone in the linear subset contains more than $5 \%$ of the data points in the original dataset, then a pure cut version is employed.

INPUT: A dataset consisting of data points from two distinct classes: $\mathrm{A}$ and $B$

1) Attempt to create an EF Class Isolation Pure Cut Hyperplane and an EF Mixed Isolation Pure Cut Hyperplane, and note the KNN subset metric for each method.

2) If no candidate hyperplanes are created:

a. Attempt to create a hyperplane using the Fallback method. If this fails, return a NULL hyperplane and exit.

3) If only one candidate hyperplane is created:

b. The candidate's method becomes the method selected, move to step 5

4) If two candidate hyperplanes are created:

c. Select the candidate with the larger KNN subset metric.

d. Move to step 5

5) Create a greedy version of the Edge Following method selected.

6) For Policies 20, 21 and 22: If the greedy version used more iterations than allowed, keep the pure cut version, otherwise return the greedy cut version

7) For Policy 23: If the greedy version of the Edge Following method has a mixed zone containing more than $5 \%$ of the total data points, return the pure cut version of the Edge Following method, otherwise return the greedy cut version.

OUTPUT: A hyperplane, and the identity of the Edge Following method used to create it Algorithm 8: Fully dynamic policies

\subsubsection{Tree Growth Termination Within Policies}

Once a tree's construction is halted by its stopping criterion, the Edge Following methods will often leave a tree which separates data points into 3 distinct groups:

1. A pure or nearly pure zone containing class A data points

2. A pure or nearly pure zone containing class B data points

3. A highly mixed zone containing class A and class B data points

The mixed zone groups need to be dealt with; a decision needs to be made as to what class to assign to the various data points in these mixed zones. The obvious way to sort 
the mixed zones out is to employ a greedy hyperplane placement method to assign classes to the various points.

Simply employing a greedy hyperplane placement method on the leaf nodes that contain a mixed group of data points is a violation of the tree's stopping criterion. Instead, the parent of every leaf whose sibling node is also a leaf is revisited. A greedy hyperplane is created for that parent node, and its performance is compared to the performance of the non-greedy hyperplane that is already in place. If the greedy hyperplane performs better, then it replaces the non-greedy hyperplane, the two old leaves are removed, and a new pair of leaves replaces them. 


\section{Experimental Setup}

\subsection{Hardware}

The hardware used consisted of two separate machines:

1) Pentium Core2Quad $2.4 \mathrm{GHZ}$, with 4 Gigabytes of RAM

2) Pentium Core2Quad 2.4 GHZ, with 8 Gigabytes of RAM

The datasets processed in the following experiments were split between the two machines, with any one dataset being fully processed by only one machine to ensure that there would be a valid basis for comparison between the different branching techniques.

\subsection{Software}

Visual Studio .NET 2008 Professional Edition (Microsoft, 2008) was used to develop an application to implement a tree creation program that applies the various hyperplane placement methods discussed in this thesis. The user interface, data objects, and tree objects were developed using Visual Basic .NET.

The actual hyperplane placement algorithm was implemented as a C++ library, and makes use of Forrest's (2009) LP solving library CLP. CLP has numerous parameters that can be set, but the only parameter that was modified from the default setting was Perturbation, which was set at 100 (indicating that the library was free to employ any perturbation it saw fit).

All results, including trees, creation times, and performance metrics were stored in an MSSQL Server 2000 database. 


\subsection{Datasets}

\subsubsection{Dataset Restrictions}

Complete Data Present: Incomplete records were removed from datasets before use.

No Categorical Data: Only datasets consisting entirely of numerical data that expresses a scale of some sort were allowed. No categorical data such as "eye color" or "vehicle type". These attributes have no continuous measurable space in which to be measured. It is important to distinguish these types of predictors from integer predictors in continuous space. A predictor of "IQ" might be permitted to contain integer values only, but the values themselves have some meaning relative to each other. Even when assigning integer values to an attribute such as eye color, the data has no meaning in relation to the different measurements taken.

Number of Samples: There is no hard definition of what qualifies a dataset as being "too small". Clearly 50 points is too small to extract reliable information from a dataset, especially when using tenfold cross validation. In the experiments performed in this thesis, datasets of less than 150 data points were not used. Due to resource limitations during testing, some of the extremely large datasets required too much time or RAM and were removed from consideration.

Corrupt Format: Some datasets have a format that make it impossible to distinguish one record from another. These datasets were removed from consideration.

Perfect Classification: Some datasets were so simple that all policies and algorithms achieved perfect classification. These datasets were removed from consideration. 


\subsubsection{UCI Repository}

All of the datasets employed in the various experiments were taken from the UCI dataset repository (University of California at Irvine, 2009). UCI datasets have become the standard sets used to test data classifiers, whether those classifiers are neural networks, statistically based classifiers, or classification trees. These datasets consist of data gathered in various studies on all sorts of real-world issues. Datasets include information on such topics as chemical analysis, disease prevalence, hand writing features, and chess end game theory. The fact that these datasets are all taken from real life situations, as opposed to being artificially created, the number of samples taken, and the breadth of different fields covered, makes the UCI datasets ideal for testing almost any classifier one might create.

Table 6 details the 35 datasets chosen for use in this thesis. Those datasets that are shown in bold were also used by Moghrabi (2003) for the purpose of testing his decision tree classifiers. The results achieved on these common datasets will be used to compare the results achieved in this thesis to his. 
Table 6: UCI datasets used in this thesis

\begin{tabular}{|c|c|c|c|c|c|c|c|c|}
\hline Dataset & $\begin{array}{l}\text { Total } \\
\text { Points } \\
\end{array}$ & $\begin{array}{l}\text { Points } \\
\text { Missing } \\
\text { data }\end{array}$ & $\begin{array}{l}\text { Net } \\
\text { Points }\end{array}$ & $\begin{array}{l}\text { Number } \\
\text { of Class } \\
0 \text { pts }\end{array}$ & $\begin{array}{l}\text { Class } 0 \\
\text { Category }\end{array}$ & $\begin{array}{l}\text { Number } \\
\text { of Class } \\
1 \text { pts }\end{array}$ & $\begin{array}{l}\text { Class } 1 \\
\text { Category }\end{array}$ & predictors \\
\hline Allbp & 2801 & 158 & 2643 & 2515 & negative & 128 & others & 21 \\
\hline Allhyper & 2800 & 157 & 2643 & 2568 & negative & 75 & others & 21 \\
\hline Allhypo & 2800 & 157 & 2643 & 2427 & negative & 216 & others & 21 \\
\hline allrep & 2800 & 157 & 2643 & 2568 & negative & 75 & others & 21 \\
\hline $\begin{array}{l}\text { balance- } \\
\text { scale }^{*}\end{array}$ & 625 & $\mathbf{0}$ & 625 & 576 & others & 49 & balanced & 4 \\
\hline $\begin{array}{l}\text { breast- } \\
\text { cancer- } \\
\text { wisconsin }\end{array}$ & 699 & 16 & 683 & 444 & benign & 239 & malig & 9 \\
\hline bupa $^{*}$ & 345 & 0 & 345 & 145 & 1 & 200 & 2 & 6 \\
\hline dermatology & 366 & 8 & 358 & 247 & others & 111 & 1 & 34 \\
\hline ecoli $^{*}$ & 336 & $\mathbf{0}$ & 336 & 193 & others & 143 & cp & 7 \\
\hline glass $^{*}$ & 214 & $\mathbf{0}$ & 214 & 163 & window & 51 & $\begin{array}{c}\begin{array}{c}\text { non- } \\
\text { window }\end{array} \\
\end{array}$ & 9 \\
\hline haberman ${ }^{*}$ & 306 & $\mathbf{0}$ & 306 & 81 & 2 & 225 & 1 & 3 \\
\hline $\begin{array}{l}\text { hd- } \\
\text { hungarian }\end{array}$ & 294 & 33 & 261 & 163 & 0 & 98 & others & 13 \\
\hline heart ${ }^{*}$ & 270 & $\mathbf{0}$ & 270 & 120 & 2 & 150 & 1 & 19 \\
\hline $\begin{array}{l}\text { house-votes- } \\
84^{*}\end{array}$ & 435 & 203 & 232 & 124 & rep & 108 & dem & 16 \\
\hline hypothyroid & 3163 & 1163 & 2000 & 1878 & negative & 122 & positive & 18 \\
\hline ionosphere & 351 & $\mathbf{0}$ & 351 & 126 & bad & 225 & good & 33 \\
\hline $\begin{array}{l}\text { letter- } \\
\text { recognition }\end{array}$ & 2329 & $\mathbf{0}$ & 2329 & 1516 & Vor W & 813 & $\mathbf{U}$ & 16 \\
\hline monks-1 ${ }^{*}$ & 556 & $\mathbf{0}$ & 556 & 278 & 0 & 278 & 1 & 6 \\
\hline monks-2 ${ }^{*}$ & 601 & 0 & 601 & 395 & $\mathbf{0}$ & 206 & 1 & 6 \\
\hline monks-3 $3^{*}$ & 554 & $\mathbf{0}$ & 554 & 266 & $\mathbf{0}$ & 288 & 1 & 6 \\
\hline new-thyroid" & 215 & $\mathbf{0}$ & 215 & 150 & normal & 65 & others & 5 \\
\hline parkinsons & 197 & 3 & 194 & 47 & 0 & 147 & 1 & 22 \\
\hline $\begin{array}{l}\text { pima- } \\
\text { indians- } \\
\text { diabetes }\end{array}$ & 768 & $\mathbf{0}$ & 768 & 500 & $\mathbf{0}$ & 268 & 1 & 8 \\
\hline $\begin{array}{l}\text { sick- } \\
\text { euthyroid }\end{array}$ & 3163 & 1163 & 2000 & 1762 & negative & 238 & others & 18 \\
\hline sonar & 208 & 0 & 208 & 111 & $\mathbf{m}$ & 97 & $\mathbf{r}$ & 60 \\
\hline tic-tac-toe & 958 & $\mathbf{0}$ & 958 & 332 & negative & 626 & positive & 18 \\
\hline vehicle & 946 & 100 & 846 & 628 & others & 218 & bus & 18 \\
\hline wdbc & 569 & $\mathbf{0}$ & 569 & 357 & benign & 212 & malig & 30 \\
\hline winel ${ }^{*}$ & 178 & 0 & 178 & 59 & 1 & 119 & others & 13 \\
\hline wine $2^{*}$ & 178 & 0 & 178 & 71 & 2 & 107 & others & 13 \\
\hline wine $3^{*}$ & 178 & 0 & 178 & 48 & 3 & 130 & others & 13 \\
\hline
\end{tabular}




\begin{tabular}{|l|c|c|c|c|c|c|c|c|}
\hline & $\begin{array}{l}\text { Total } \\
\text { Points }\end{array}$ & $\begin{array}{l}\text { Points } \\
\text { Missing } \\
\text { data }\end{array}$ & $\begin{array}{l}\text { Net } \\
\text { Points }\end{array}$ & $\begin{array}{l}\text { Number } \\
\text { of Class } \\
\text { 0 pts }\end{array}$ & $\begin{array}{l}\text { Class 0 } \\
\text { Category }\end{array}$ & $\begin{array}{l}\text { Number } \\
\text { of Class } \\
\text { 1 pts }\end{array}$ & $\begin{array}{l}\text { Class 1 } \\
\text { Category }\end{array}$ & predictors \\
\hline wpbc & 194 & 0 & 194 & 148 & n & 46 & r & 33 \\
\hline yeastcyt & $\mathbf{1 4 8 4}$ & $\mathbf{0}$ & $\mathbf{1 4 8 4}$ & $\mathbf{4 6 3}$ & cyt & $\mathbf{1 0 2 1}$ & others & $\mathbf{8}$ \\
\hline yeastnuc & 1484 & 0 & 1484 & 1055 & others & 429 & nuc & 8 \\
\hline yeastother & 1484 & 0 & 1484 & 892 & $\begin{array}{l}\text { nuc or } \\
\text { cyt }\end{array}$ & 592 & others & 8 \\
\hline
\end{tabular}

Note: Datasets marked with a "*" were used in the first half of the experiment.

\section{Rejected UCI Data Sets}

The UCI repository (University of California at Irvine, 1987) has 118

classification dataset sources. Some sources contain directories with multiple data sets. From these 118 sources, 50 datasets were found that had neither categorical data nor corruption problems.

Of the 50 datasets initially taken, the following datasets were removed due to the fact they needed more resources than were available in order to process them: abalone, clean1, clean2, cmc, eighthr, hillvalleywithnoise, hillvalleywithoutnoise, magic, onehr, optdigits, pageblocks, sat, segmentation, and spambase. The iris dataset was removed due to the fact that all classifiers were able to perfectly classify the data in this dataset.

\subsection{Parameter Settings}

The various hyperplane placement and tree creation methods make use of several parameters that are available for modification by the user.

The Edge Following hyperplane placement methods make use of several parameters, the following values were used:

- $\mathrm{K}_{\mathrm{mz}}=20$

- $\mathrm{K}_{\mathrm{mzt}}=10$

- Minimum dataset size permitted for an EF method to be applied: 40 . 
- $\quad$ Minimum KNN subset metric allowed: 4

Notes on the Edge Following parameters:

- The minimum dataset size was somewhat arbitrary, obviously there must be at least $\mathrm{K}_{\mathrm{mz}}$ data points in a dataset to use an $\mathrm{EF}$ method, otherwise there would not be enough neighbors present to determine whether a data point is in the $\mathrm{KNN}$ subset. Clearly if there are only $\mathrm{K}_{\mathrm{mz}}$ data points present then all data points will be included in the KNN subset, which would make applying an EF method pointless. A setting of $2 * \mathrm{~K}_{\mathrm{mz}}=40$ was decided upon.

- A KNN subset metric of less than 4 indicates that a dataset is so heavily mixed, or so pure, that less than 4 data points were separated out when placing a pure cut on the KNN subset. Such a dataset indicates that the node should either be terminated as a leaf, or be subdivided using a greedy hyperplane placement method.

The only stopping criterion used stipulated that a minimum of 5 data points be present in order for further subdivision to be attempted. No pruning was employed.

\subsection{Metrics}

All experiments were performed using 10 fold cross validation (see Section 2.2). The experimental results list the average accuracy and standard deviation across the 10 folds. Comparisons of the various policies are also made using performance profiles. 


\subsubsection{Performance Profiles}

The concept of performance profiles was developed by Dolan and More (2002) as a way of benchmarking optimization software.

Given $D$ datasets, and $H$ tree development policies $a_{h, d}$ represents the accuracy a tree development policy $h$ achieves on a given dataset $d$. The performance ratio is defined as:

$$
r_{h, d}=\frac{a_{h, d}}{\max \left\{\mathrm{a}_{\mathrm{h}, \mathrm{d}}: \mathrm{h} \in \mathrm{H}\right\}}
$$

So for any given dataset, the performance ratio for each policy represents the ratio of the accuracy of that given policy to the accuracy of the best performing policy.

The performance of a given group of policies, even when presented as performance ratios, on any given dataset does not give an accurate depiction of their relative worth. A comparison over many datasets is needed. The distribution function of the performance ratios is referred to as a performance profile and is defined as:

$$
\rho_{h}(\tau)=\frac{1}{n_{d}} \operatorname{size}\left\{d \in D: r_{h, d} \geq \tau\right\}
$$

and represents the probability that a tree development policy $h$ will be within a factor $\tau$ of the best result. D represents the set of datasets used, and $n_{d}$ represents the number of datasets. 


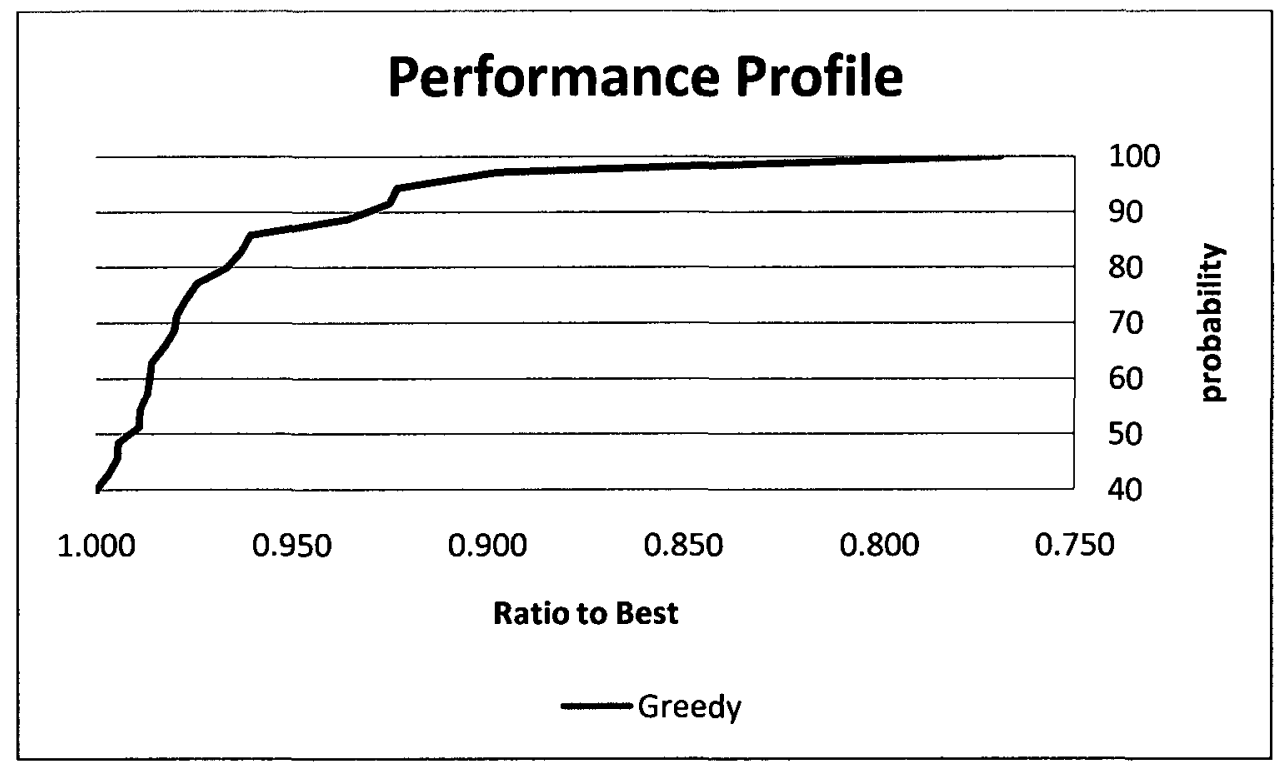

Figure 41: An example of a performance profile.

Figure 41 is an example of the format in which performance profiles are presented in this thesis. The x-axis, labeled as "Ratio to Best", represents the possible values of $\tau$, and the y-axis, labeled as "Probability" represents the possible values of $\rho_{h}(\tau)$.

When comparing classifiers, care must be taken to define what is meant by the word "better". The "No free lunch" principle (Duda and Hart, 1973) essentially states that there is no such thing as a classifier that will outperform all other classifiers on all datasets. The use of performance profiles allows the performance of various classifiers to be compared across many datasets. In this thesis, when one classifier is said to have performed better than another, this means that its test results have produced a better performance profile.

When considering the performance profile of a single policy, the higher up, and further left the curve is on the plot, the better the policy's performance. When comparing the performance profiles of two policies, one policy is said to be "better" if the majority 
of its performance profile is above and to the left of the other policy's performance profile.

\subsection{Algorithms Tested}

A quick test of 3 greedy hyperplane placement methods (accuracy, Gini and EROC) against all 35 datasets, summarized in Table 7, showed that the difference in performance between these three greedy methods was quite small. Given the small difference in performance among these greedy methods, and the popularity of accuracy as a greedy hyperplane placement method, it was decided to use accuracy as the greedy hyperplane placement method in the experiments performed in this thesis.

Table 7: Average performance of 3 greedy hyperplane placement methods.

\begin{tabular}{|l|l|l|l|}
\hline Greedy Hyperplane Placement Method & Accuracy & Gini & EROC \\
\hline Average Accuracy & $\mathbf{0 . 8 8}$ & $\mathbf{0 . 8 8 3}$ & 0.876 \\
\hline
\end{tabular}

Due to the large number of policies, the experimental process to test their effectiveness was split into two steps.

The first step of the experiment involved testing all 23 policies, along with a greedy hyperplane placement algorithm, on the smallest 15 data sets. These datasets are marked with an "“"” beside their names in Table 6. The results from this experiment were used to reduce the number of policies that were tested in the second step of the experiment.

The second step of the experiment consisted of processing each dataset using the policies selected in Step 1 as well as a Greedy hyperplane placement algorithm. 10 fold cross validation is used. The average performance and standard deviation across the 10 folds is then taken as representative of the policies performance on the various datasets. 
The results of this experiment were then compared to the results documented in Moghrabi's (2003) thesis, and to the performance of the following commercial classifiers:

- C4.5 (Quinlan 1993)

- Cart 6.0 (Salford Systems, 2009)

- OC1 (Murthy, 1997)

- GATree (Athanassios and Dimitrios, 2001) 


\section{Experimental Results}

The goal of the experiment is to document the performance of the policies developed in this thesis. These policies are compared to an implementation of Chinneck's (2009) greedy hyperplane placement method, and other state of the art methods.

The first step in the experiment involved testing all 23 policies, along with a greedy hyperplane placement algorithm that maximizes for accuracy, on 15 of the datasets. The performance of these policies was measured in terms of accuracy, and the best performing fixed order policies, partially dynamic policies and fully dynamic policies were then chosen and used in the second step of the experiment.

Instead of presenting numerous tables requiring significant interpretation, the results of the first step in the experiment are summarized in the performance profiles shown in Figure 42 to Figure 44. 


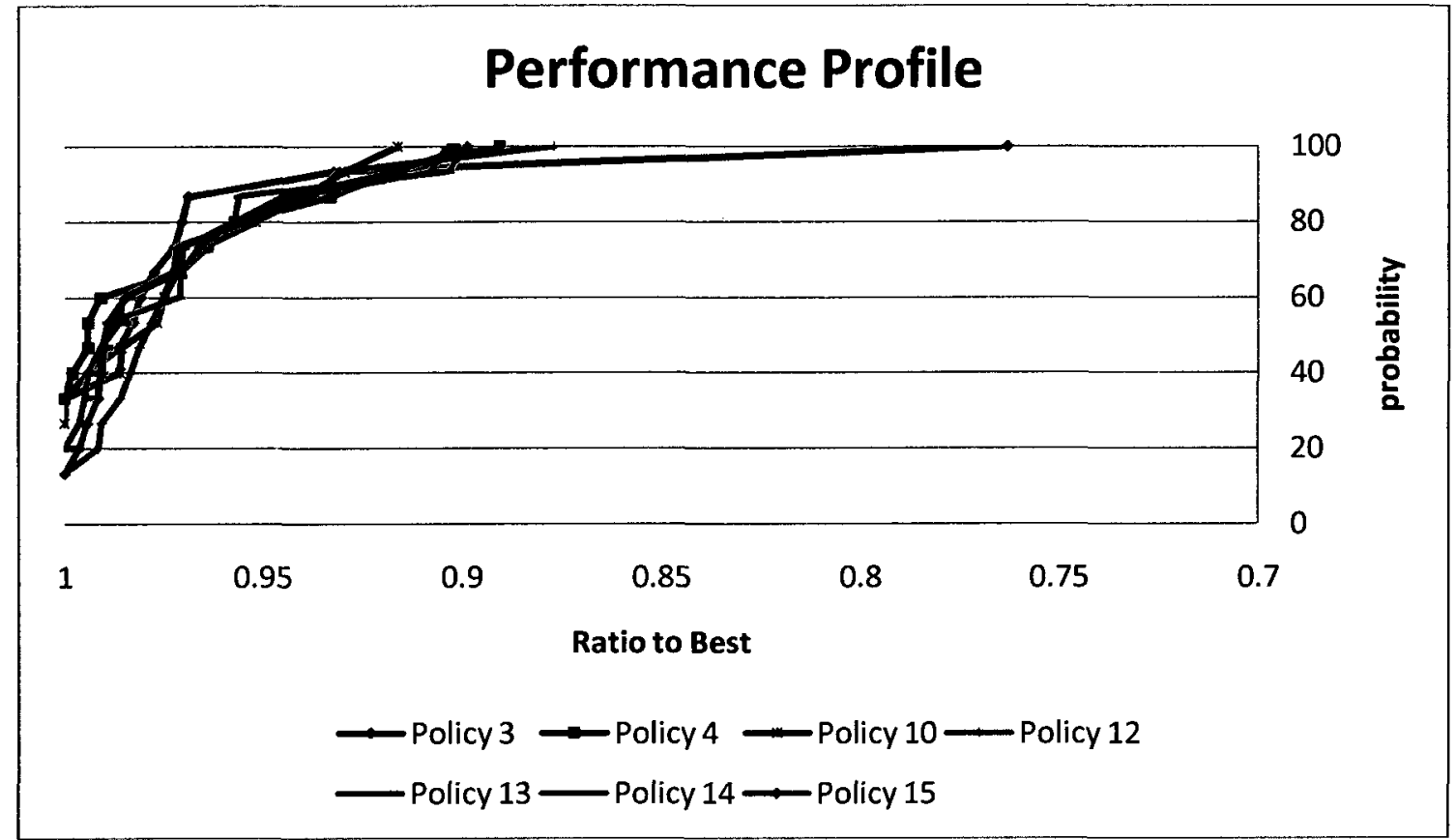

Figure 42: The performance profile for the best 7 fixed order policies.

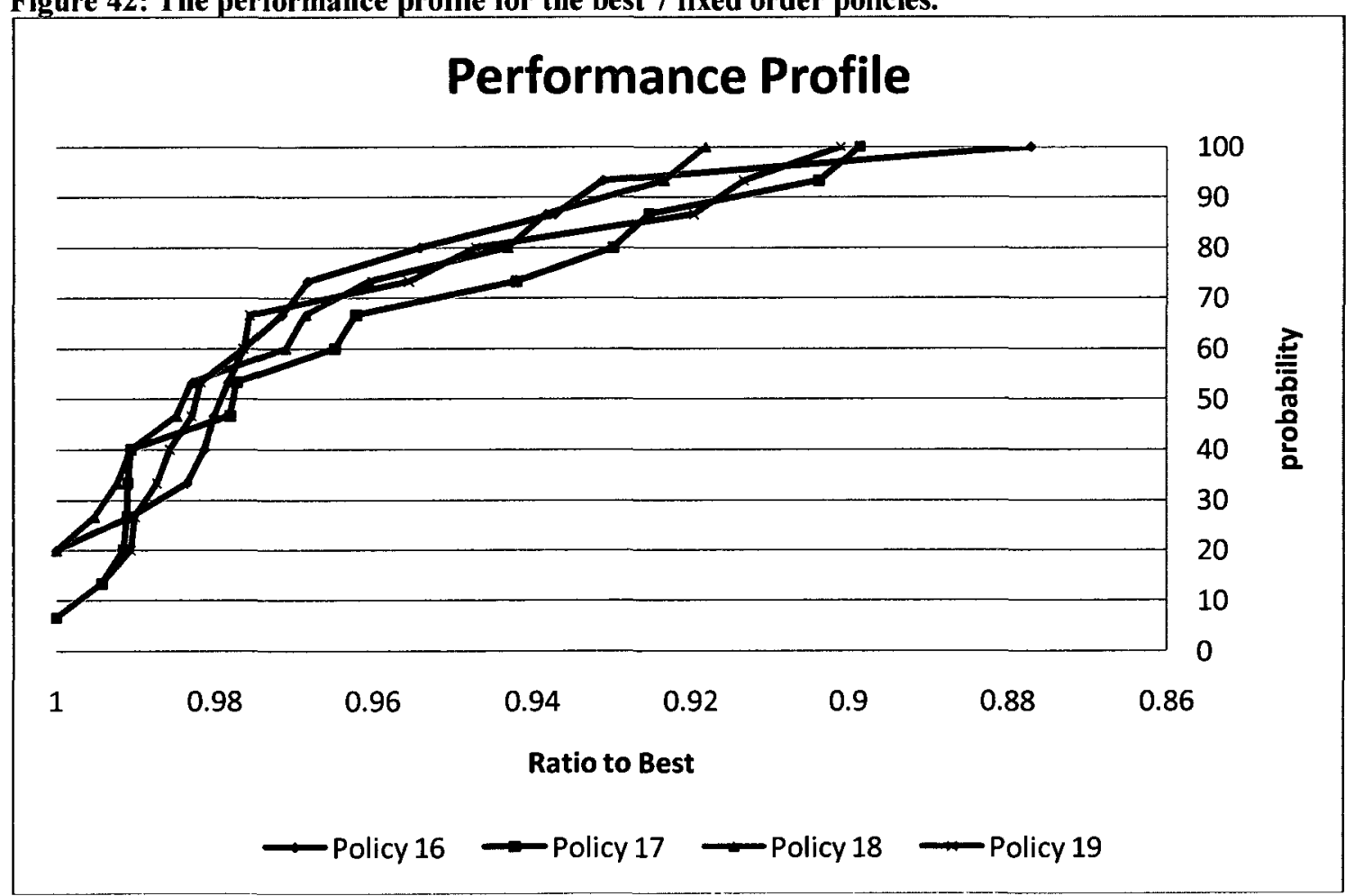

Figure 43: The Performance Profile for the 4 partially dynamic policies. 


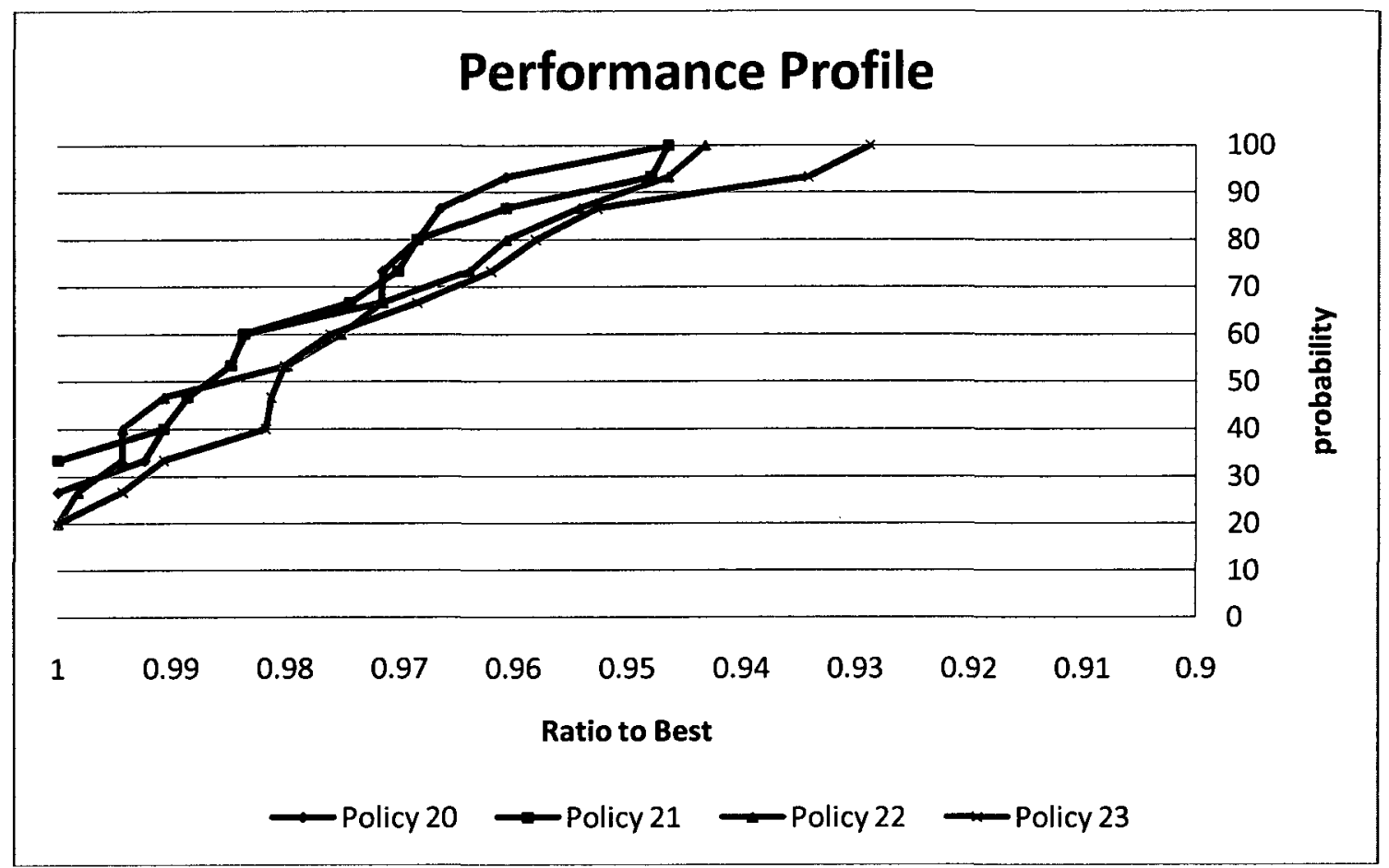

Figure 44: The Performance Profile for the fully dynamic policies.

Due to the large number of fixed order policies, 4 were chosen for use in the second half of the experiment. Determining the "best" 4 fixed order policies is difficult. An examination of Figure 42 shows that the best 7 policies are fairly evenly matched. Policies 3 and 4 clearly distinguish themselves, and after much consideration, 10 and 13 were decided upon as the remaining two candidates.

The partially dynamic and fully dynamic policies were easier to adjudicate. An examination of Figure 43 shows that policies 16 and 18 outperform policies 17 and 19, and an examination of Figure 44 shows that Policies 20 and 21 outperform Policies 22 and 23 .

The second step in the experiment involves taking the policies selected in the first step of the experiment and running them, along with a greedy hyperplane placement algorithm (again maximizing for accuracy), against all 35 datasets. Table 8 summarizes the results. For each dataset listed, the "avg" row represents the average accuracy of the 
10 folds, and the "SD" row represents the standard deviation. The bolded entries highlight the highest accuracy achieved for a given dataset. Some rows in Table 8 contain what appear to be ties for the best performing Policy, and have one metric highlighted while others are not. This is due to the fact that results in Table 8 are truncated to 3 digits in order to fit the results to the page. Two scores that may appear to be a tie may in actual fact be slightly different. Table 9 lists the average accuracy and standard deviation from the results compiled.

Table 8: Experimental Results

\begin{tabular}{|c|c|c|c|c|c|c|c|c|c|c|}
\hline Data Sets & & Greedy & $\begin{array}{c}\text { Policy } \\
3 \\
\end{array}$ & $\begin{array}{c}\text { Policy } \\
4 \\
\end{array}$ & $\begin{array}{c}\text { Policy } \\
10 \\
\end{array}$ & $\begin{array}{c}\text { Policy } \\
13 \\
\end{array}$ & $\begin{array}{c}\text { Policy } \\
16 \\
\end{array}$ & $\begin{array}{c}\text { Policy } \\
18 \\
\end{array}$ & $\begin{array}{c}\text { Policy } \\
20 \\
\end{array}$ & $\begin{array}{c}\text { Policy } \\
21 \\
\end{array}$ \\
\hline \multirow[t]{2}{*}{ allbp } & Avg & 0.967 & 0.967 & 0.960 & 0.962 & 0.963 & 0.967 & 0.955 & 0.957 & 0.956 \\
\hline & SD & 0.008 & 0.008 & 0.008 & 0.014 & 0.008 & 0.010 & 0.010 & 0.010 & 0.010 \\
\hline \multirow[t]{2}{*}{ allhyper } & Avg & 0.981 & 0.978 & 0.979 & 0.977 & 0.978 & 0.974 & 0.979 & 0.976 & 0.975 \\
\hline & SD & 0.009 & 0.010 & 0.006 & 0.009 & 0.006 & 0.011 & 0.013 & 0.006 & 0.008 \\
\hline \multirow[t]{2}{*}{ allhypo } & Avg & 0.985 & 0.985 & 0.980 & 0.972 & 0.980 & 0.977 & 0.971 & 0.978 & 0.977 \\
\hline & $\mathrm{SD}$ & 0.006 & 0.006 & 0.010 & 0.014 & 0.010 & 0.011 & 0.016 & 0.009 & 0.009 \\
\hline \multirow[t]{2}{*}{ allirep } & Avg & 0.978 & 0.977 & 0.989 & 0.975 & 0.986 & 0.982 & 0.974 & 0.984 & 0.984 \\
\hline & SD & 0.012 & 0.012 & 0.006 & 0.006 & 0.006 & 0.004 & 0.004 & 0.007 & 0.007 \\
\hline \multirow[t]{2}{*}{$\begin{array}{l}\text { balance- } \\
\text { scale }\end{array}$} & Avg & 0.922 & 0.922 & 0.901 & 0.856 & 0.909 & 0.917 & 0.844 & 0.934 & 0.934 \\
\hline & SD & 0.026 & 0.026 & 0.029 & 0.081 & 0.035 & 0.031 & 0.073 & 0.027 & 0.027 \\
\hline \multirow[t]{2}{*}{$\begin{array}{l}\text { breast- } \\
\text { cancer- } \\
\text { wisconsin }\end{array}$} & Avg & 0.963 & 0.963 & 0.944 & 0.959 & 0.947 & 0.949 & 0.955 & 0.940 & 0.943 \\
\hline & SD & 0.023 & 0.023 & 0.023 & 0.024 & 0.024 & 0.027 & 0.023 & 0.029 & 0.027 \\
\hline \multirow[t]{2}{*}{ bupa } & Avg & 0.676 & 0.676 & 0.655 & 0.664 & 0.649 & 0.685 & 0.667 & 0.681 & 0.681 \\
\hline & $\mathrm{SD}$ & 0.081 & 0.081 & 0.075 & 0.095 & 0.072 & 0.090 & 0.093 & 0.083 & 0.083 \\
\hline \multirow[t]{2}{*}{ dermatology } & Avg & 0.980 & 0.986 & 0.983 & 0.986 & 0.983 & 0.980 & 0.983 & 0.980 & 0.980 \\
\hline & $\mathrm{SD}$ & 0.022 & 0.019 & 0.019 & 0.019 & 0.019 & 0.018 & 0.019 & 0.013 & 0.018 \\
\hline \multirow[t]{2}{*}{ ecoli } & Avg & 0.961 & 0.961 & 0.961 & 0.934 & 0.923 & 0.949 & 0.952 & 0.949 & 0.949 \\
\hline & SD & 0.027 & 0.027 & 0.027 & 0.048 & 0.052 & 0.019 & 0.020 & 0.019 & 0.019 \\
\hline \multirow[t]{2}{*}{ glass } & Avg & 0.902 & 0.916 & 0.907 & 0.921 & 0.939 & 0.898 & 0.921 & 0.884 & 0.884 \\
\hline & SD & 0.060 & 0.046 & 0.060 & 0.036 & 0.042 & 0.044 & 0.030 & 0.084 & 0.084 \\
\hline haberman & Avg & 0.732 & 0.732 & 0.697 & 0.660 & 0.710 & 0.723 & 0.661 & 0.700 & 0.700 \\
\hline
\end{tabular}




\begin{tabular}{|c|c|c|c|c|c|c|c|c|c|c|}
\hline Data Sets & & Greedy & $\begin{array}{c}\text { Policy } \\
3 \\
\end{array}$ & $\begin{array}{c}\text { Policy } \\
4\end{array}$ & $\begin{array}{c}\text { Policy } \\
10\end{array}$ & $\begin{array}{c}\text { Policy } \\
13 \\
\end{array}$ & $\begin{array}{c}\text { Policy } \\
16\end{array}$ & $\begin{array}{c}\text { Policy } \\
18 \\
\end{array}$ & $\begin{array}{c}\text { Policy } \\
20 \\
\end{array}$ & $\begin{array}{c}\text { Policy } \\
21 \\
\end{array}$ \\
\hline & $\mathrm{SD}$ & 0.059 & 0.059 & 0.121 & 0.093 & 0.111 & 0.048 & 0.094 & 0.089 & 0.089 \\
\hline \multirow[t]{2}{*}{$\begin{array}{l}\text { hd- } \\
\text { hungarian }\end{array}$} & Avg & 0.774 & 0.781 & 0.728 & 0.778 & 0.728 & 0.763 & 0.774 & 0.801 & 0.801 \\
\hline & $\mathrm{SD}$ & 0.064 & 0.083 & 0.067 & 0.083 & 0.080 & 0.088 & 0.079 & 0.074 & 0.074 \\
\hline \multirow[t]{2}{*}{ heart } & Avg & 0.733 & 0.737 & 0.789 & 0.726 & 0.781 & 0.730 & 0.737 & 0.793 & 0.763 \\
\hline & SD & 0.059 & 0.063 & 0.066 & 0.055 & 0.077 & 0.053 & 0.054 & 0.074 & 0.073 \\
\hline \multirow[t]{2}{*}{$\begin{array}{l}\text { house- } \\
\text { votes- } 84\end{array}$} & Avg & 0.931 & 0.931 & 0.939 & 0.939 & 0.953 & 0.892 & 0.936 & 0.900 & 0.887 \\
\hline & $\mathrm{SD}$ & 0.056 & 0.056 & 0.040 & 0.044 & 0.045 & 0.063 & 0.063 & 0.059 & 0.059 \\
\hline \multirow[t]{2}{*}{ hypothyroid } & Avg & 0.979 & 0.979 & 0.979 & 0.975 & 0.974 & 0.967 & 0.971 & 0.972 & 0.974 \\
\hline & $\mathrm{SD}$ & 0.009 & 0.009 & 0.008 & 0.010 & 0.009 & 0.009 & 0.006 & 0.009 & 0.008 \\
\hline \multirow[t]{2}{*}{ ionosphere } & Avg & 0.858 & 0.843 & 0.846 & 0.849 & 0.840 & 0.832 & 0.855 & 0.808 & 0.803 \\
\hline & SD & 0.035 & 0.036 & 0.071 & 0.057 & 0.047 & 0.055 & 0.057 & 0.065 & 0.063 \\
\hline \multirow[t]{2}{*}{$\begin{array}{l}\text { letter- } \\
\text { recognition }\end{array}$} & Avg & 0.990 & 0.990 & 0.990 & 0.990 & 0.990 & 0.971 & 0.974 & 0.983 & 0.991 \\
\hline & $\mathrm{SD}$ & 0.005 & 0.005 & 0.006 & 0.005 & 0.006 & 0.014 & 0.008 & 0.007 & 0.006 \\
\hline \multirow[t]{2}{*}{ monks-1 } & Avg & 0.898 & 1.000 & 1.000 & 1.000 & 1.000 & 0.991 & 0.987 & 1.000 & 1.000 \\
\hline & SD & 0.077 & 0.000 & 0.000 & 0.000 & 0.000 & 0.019 & 0.028 & 0.000 & 0.000 \\
\hline \multirow[t]{2}{*}{ monks-2 } & Avg & 0.659 & 0.659 & 0.775 & 0.784 & 0.782 & 0.815 & 0.754 & 0.857 & 0.857 \\
\hline & $\mathrm{SD}$ & 0.070 & 0.070 & 0.058 & 0.051 & 0.064 & 0.048 & 0.096 & 0.038 & 0.038 \\
\hline \multirow[t]{2}{*}{ monks-3 } & Avg & 0.910 & 0.946 & 0.975 & 0.986 & 0.968 & 0.971 & 0.978 & 0.971 & 0.968 \\
\hline & SD & 0.065 & 0.059 & 0.020 & 0.018 & 0.019 & 0.023 & 0.021 & 0.033 & 0.032 \\
\hline \multirow[t]{2}{*}{ new-thyroid } & Avg & 0.911 & 0.879 & 0.930 & 0.892 & 0.907 & 0.892 & 0.874 & 0.916 & 0.888 \\
\hline & SD & 0.067 & 0.069 & 0.064 & 0.082 & 0.069 & 0.080 & 0.064 & 0.069 & 0.080 \\
\hline \multirow[t]{2}{*}{ parkinsons } & Avg & 0.819 & 0.819 & 0.824 & 0.815 & 0.819 & 0.840 & 0.841 & 0.840 & 0.840 \\
\hline & SD & 0.075 & 0.077 & 0.088 & 0.122 & 0.097 & 0.103 & 0.086 & 0.087 & 0.087 \\
\hline \multirow[t]{2}{*}{$\begin{array}{l}\text { pima- } \\
\text { indians- } \\
\text { diabetes }\end{array}$} & Avg & 0.764 & 0.764 & 0.761 & 0.740 & 0.758 & 0.734 & 0.725 & 0.729 & 0.730 \\
\hline & $\mathrm{SD}$ & 0.032 & 0.032 & 0.047 & 0.043 & 0.062 & 0.065 & 0.040 & 0.030 & 0.029 \\
\hline \multirow[t]{2}{*}{$\begin{array}{l}\text { sick- } \\
\text { euthyroid }\end{array}$} & Avg & 0.953 & 0.954 & 0.942 & 0.935 & 0.954 & 0.949 & 0.941 & 0.946 & 0.945 \\
\hline & $\mathrm{SD}$ & 0.009 & 0.011 & 0.026 & 0.016 & 0.016 & 0.013 & 0.011 & 0.013 & 0.017 \\
\hline \multirow[t]{2}{*}{ sonar } & Avg & 0.712 & 0.679 & 0.716 & 0.726 & 0.722 & 0.760 & 0.673 & 0.745 & 0.735 \\
\hline & $\mathrm{SD}$ & 0.116 & 0.068 & 0.097 & 0.057 & 0.071 & 0.053 & 0.122 & 0.112 & 0.110 \\
\hline \multirow[t]{2}{*}{ tic-tac-toe } & Avg & 0.971 & 0.971 & 0.971 & 0.957 & 0.957 & 0.965 & 0.954 & 0.965 & 0.965 \\
\hline & $\mathrm{SD}$ & 0.035 & 0.035 & 0.035 & 0.028 & 0.028 & 0.033 & 0.041 & 0.033 & 0.033 \\
\hline
\end{tabular}




\begin{tabular}{|c|c|c|c|c|c|c|c|c|c|c|}
\hline Data Sets & & Greedy & $\begin{array}{c}\text { Policy } \\
3 \\
\end{array}$ & $\begin{array}{c}\text { Policy } \\
4\end{array}$ & $\begin{array}{c}\text { Policy } \\
10 \\
\end{array}$ & $\begin{array}{c}\text { Policy } \\
13 \\
\end{array}$ & $\begin{array}{c}\text { Policy } \\
16\end{array}$ & $\begin{array}{c}\text { Policy } \\
18\end{array}$ & $\begin{array}{c}\text { Policy } \\
20\end{array}$ & $\begin{array}{c}\text { Policy } \\
21\end{array}$ \\
\hline \multirow[t]{2}{*}{ vehicle } & Avg & 0.952 & 0.970 & 0.959 & 0.967 & 0.961 & 0.972 & 0.959 & 0.962 & 0.956 \\
\hline & SD & 0.027 & 0.014 & 0.013 & 0.023 & 0.018 & 0.019 & 0.027 & 0.013 & 0.032 \\
\hline \multirow[t]{2}{*}{$w d b c$} & Avg & 0.940 & 0.930 & 0.940 & 0.931 & 0.908 & 0.924 & 0.921 & 0.893 & 0.893 \\
\hline & SD & 0.023 & 0.032 & 0.023 & 0.035 & 0.030 & 0.041 & 0.038 & 0.043 & 0.043 \\
\hline \multirow[t]{2}{*}{ winel } & Avg & 0.978 & 0.978 & 0.978 & 0.922 & 0.972 & 0.944 & 0.944 & 0.983 & 0.972 \\
\hline & SD & 0.027 & 0.027 & 0.027 & 0.100 & 0.037 & 0.075 & 0.050 & 0.025 & 0.037 \\
\hline \multirow[t]{2}{*}{ wine2 } & Avg & 0.972 & 0.972 & 0.972 & 0.972 & 0.972 & 0.972 & 0.972 & 0.972 & 0.972 \\
\hline & SD & 0.045 & 0.045 & 0.045 & 0.045 & 0.045 & 0.045 & 0.045 & 0.045 & 0.045 \\
\hline \multirow[t]{2}{*}{ wine3 } & Avg & 0.967 & 0.978 & 0.967 & 0.978 & 0.967 & 0.972 & 0.978 & 0.972 & 0.972 \\
\hline & SD & 0.044 & 0.037 & 0.044 & 0.037 & 0.044 & 0.045 & 0.037 & 0.045 & 0.045 \\
\hline \multirow[t]{2}{*}{ wpbc } & Avg & 0.722 & 0.712 & 0.732 & 0.680 & 0.728 & 0.691 & 0.722 & 0.696 & 0.691 \\
\hline & SD & 0.079 & 0.103 & 0.055 & 0.095 & 0.087 & 0.100 & 0.107 & 0.104 & 0.100 \\
\hline \multirow[t]{2}{*}{ yeastcyt } & Avg & 0.706 & 0.708 & 0.733 & 0.693 & 0.708 & 0.709 & 0.708 & 0.708 & 0.708 \\
\hline & SD & 0.022 & 0.023 & 0.091 & 0.072 & 0.022 & 0.023 & 0.023 & 0.025 & 0.025 \\
\hline \multirow[t]{2}{*}{ yeastnuc } & Avg & 0.759 & 0.771 & 0.757 & 0.755 & 0.749 & 0.764 & 0.767 & 0.772 & 0.772 \\
\hline & SD & 0.042 & 0.028 & 0.039 & 0.028 & 0.029 & 0.028 & 0.031 & 0.051 & 0.051 \\
\hline \multirow[t]{2}{*}{ yeastother } & Avg & 0.810 & 0.797 & 0.807 & 0.813 & 0.811 & 0.810 & 0.797 & 0.804 & 0.806 \\
\hline & SD & 0.022 & 0.021 & 0.019 & 0.036 & 0.029 & 0.026 & 0.021 & 0.024 & 0.025 \\
\hline
\end{tabular}

Table 9: Average Accuracy and Standard Deviation over all datasets

\begin{tabular}{|l|c|c|c|c|c|c|c|c|c|}
\hline & Greedy & $\begin{array}{c}\text { Policy } \\
3\end{array}$ & $\begin{array}{c}\text { Policy } \\
4\end{array}$ & $\begin{array}{c}\text { Policy } \\
10\end{array}$ & $\begin{array}{c}\text { Policy } \\
13\end{array}$ & $\begin{array}{c}\text { Policy } \\
16\end{array}$ & $\begin{array}{c}\text { Policy } \\
18\end{array}$ & $\begin{array}{c}\text { Policy } \\
20\end{array}$ & $\begin{array}{c}\text { Policy } \\
21\end{array}$ \\
\hline $\begin{array}{l}\text { Average } \\
\text { Accuracy }\end{array}$ & 0.878 & 0.880 & $\mathbf{0 . 8 8 5}$ & 0.876 & 0.882 & 0.881 & 0.874 & 0.884 & 0.882 \\
\hline $\begin{array}{l}\text { Average } \\
\text { Standard } \\
\text { Deviation }\end{array}$ & 0.041 & 0.038 & 0.041 & 0.045 & 0.041 & 0.041 & 0.044 & 0.042 & 0.043 \\
\hline
\end{tabular}

The performance of the various tree development policies were measured relative to each other using performance profiles based on accuracy. An examination of the standard deviations did not reveal anything unusual. 


\subsection{A Comparison of Policies Vs. the Greedy Hyperplane Placement}

\section{Method}

The curves on the performance profiles shown in this section were derived from the experimental results compiled in Table 8 . Showing all 9 curves on a single graph results in a graph that is very hard to interpret, so a set of four graphs is presented, comparing 2 non-greedy policies against the greedy hyperplane placement method in each graph. Even though only 3 curves are plotted on each graph, the ratios plotted are to the best of all 9 methods tested.

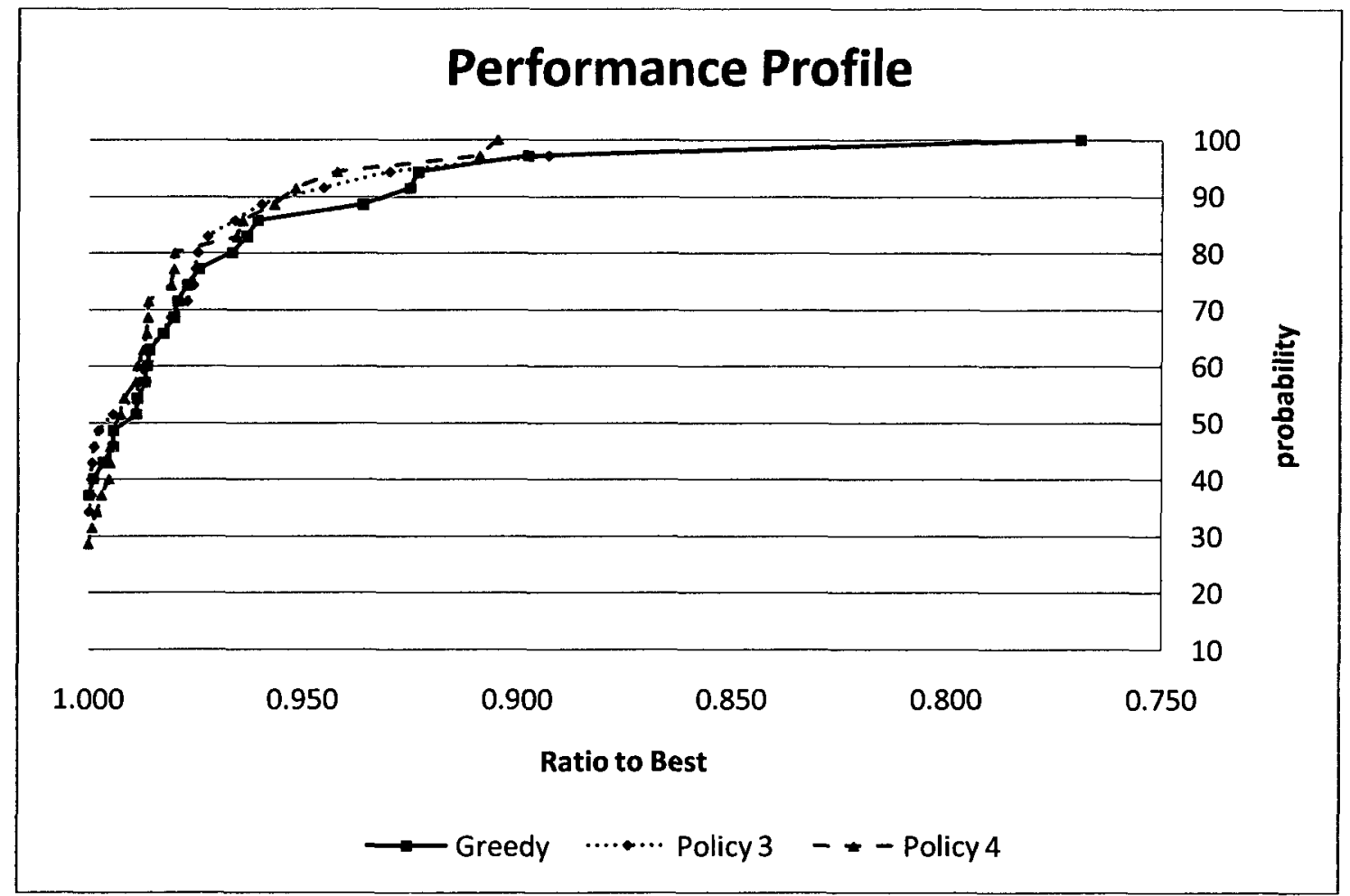

Figure 45: A performance profile plotting the performance of policies 3 and 4 against the greedy method 


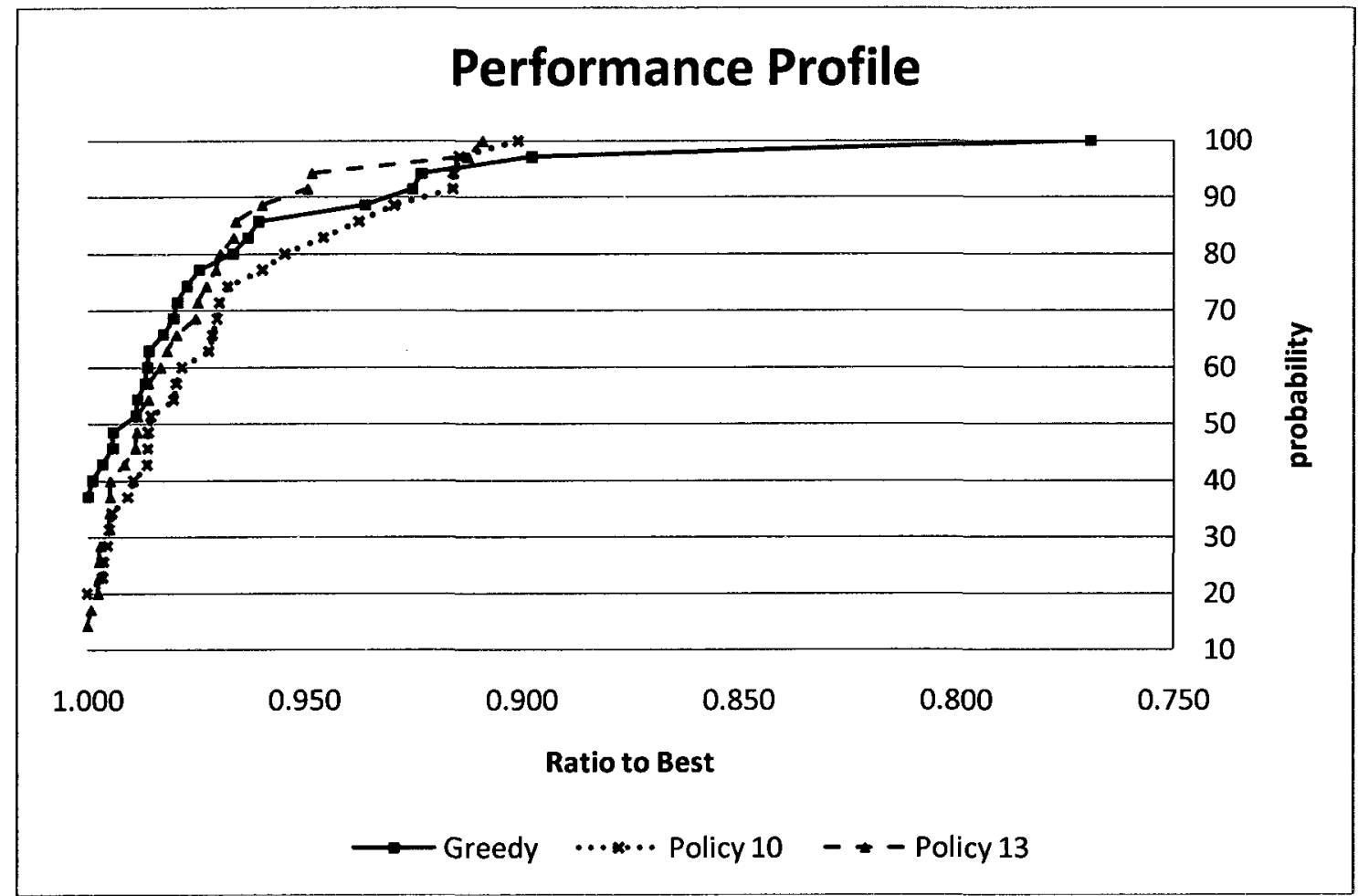

Figure 46: A performance profile plotting the performance of policies 10 and 13 against the greedy method.

Figure 45 and Figure 46 plot the performance of four Fixed Order Policies against the performance of the Greedy hyperplane placement method. Of the Fixed Order Policies, Policies 3 and 4 perform better than the Greedy hyperplane placement method. Given that curves representing the performance of Policies 10 and 13 cross the curve representing the performance of the Greedy hyperplane placement method, a determination of which is better is more difficult to make. Policy 13 performs about as well as the greedy method, while policy 10 looks to be worse than the greedy hyperplane placement method. 


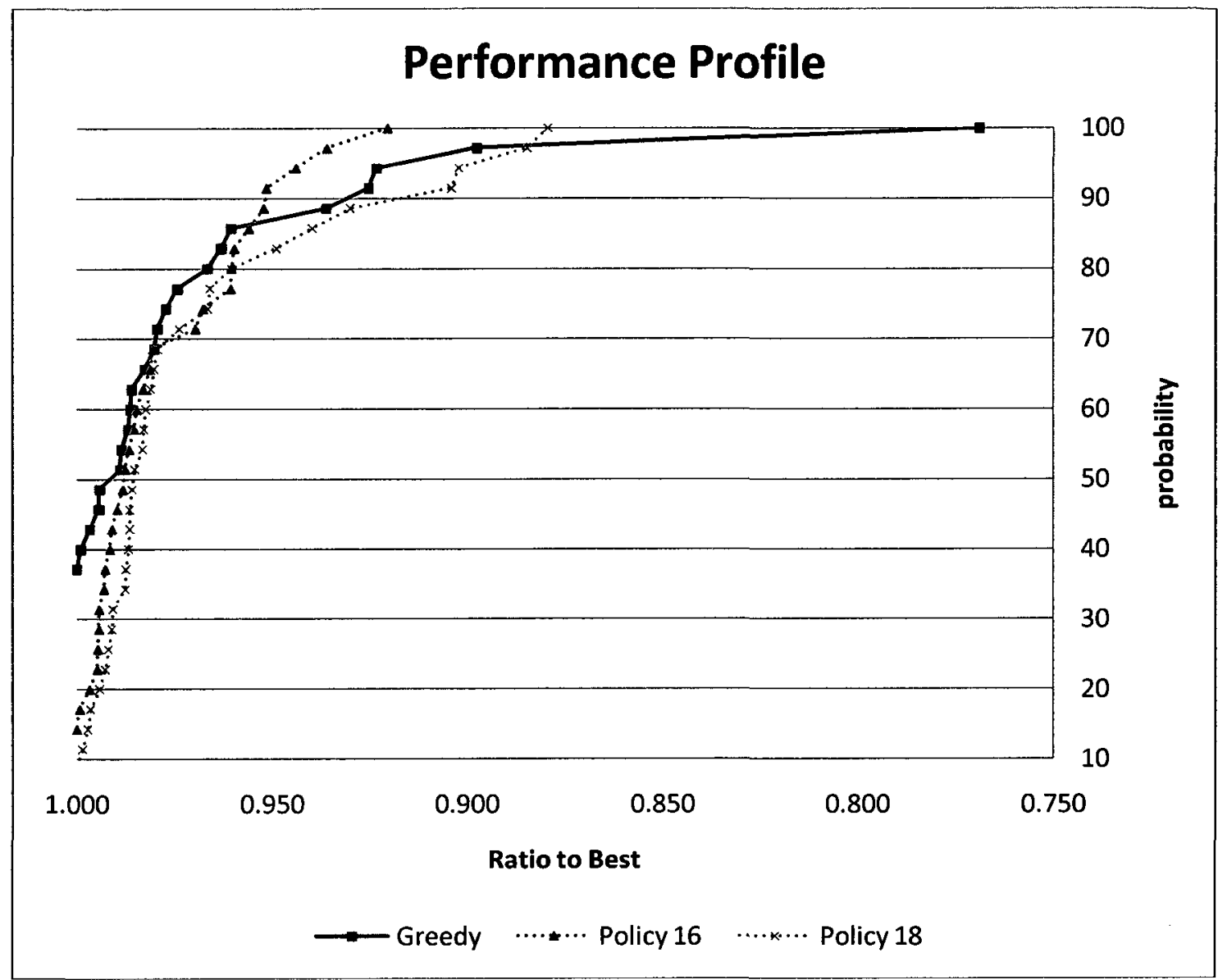

Figure 47: A performance profile plotting the performance of policies 16 and 18 against the greedy method.

Figure 47 plots the performance of two Partially Dynamic Policies against the performance of the Greedy hyperplane placement method. The partially dynamic policies' curves intersect with the greedy method's curve. Policy 16 performs roughly as well as the greedy method, but Policy 18 is clearly worse than the greedy method. 


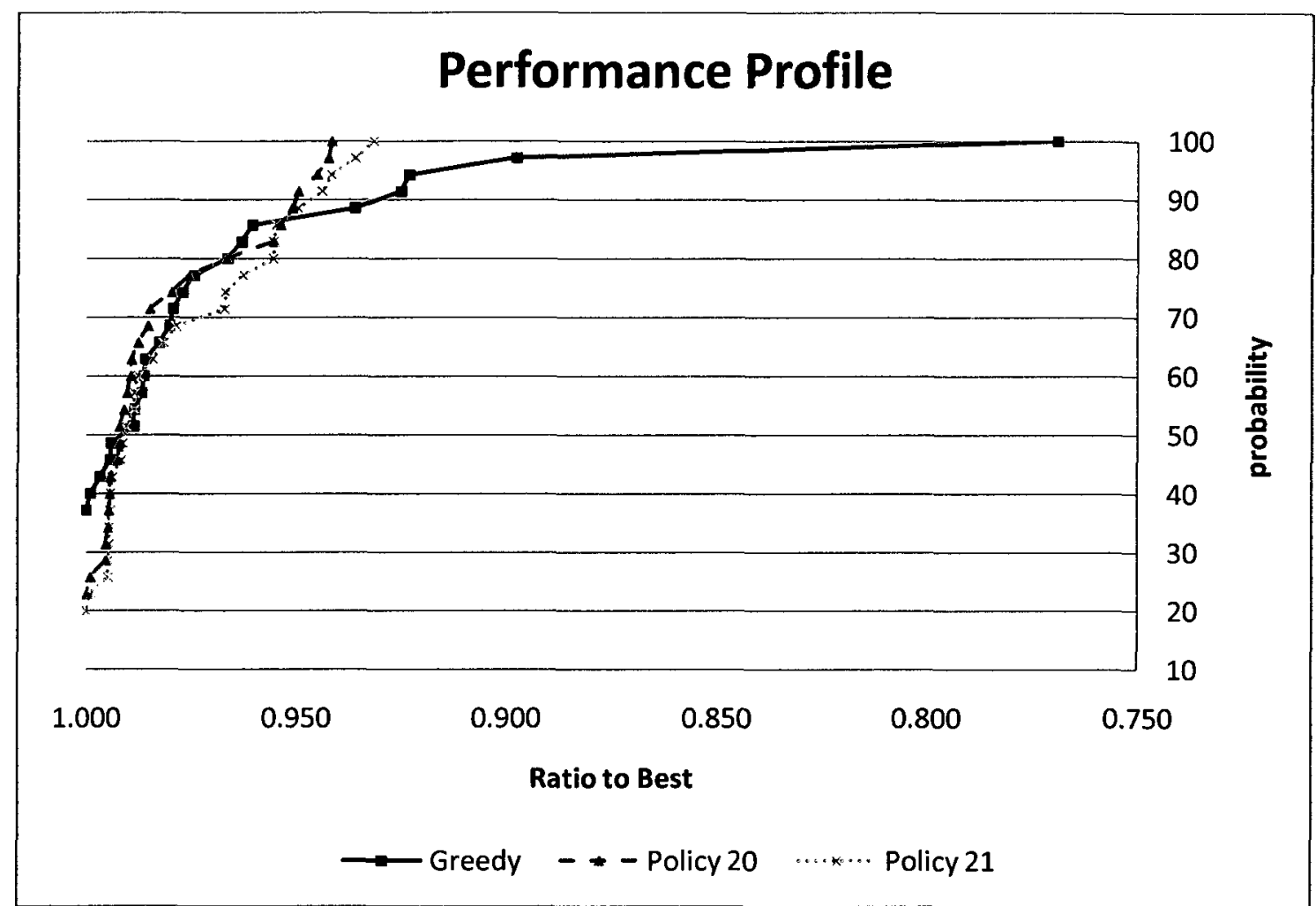

Figure 48: A performance profile plotting the performance of policies 20 and 21 against the greedy method.

Figure 48 plots the performance of two Fully Dynamic Policies against the performance of the Greedy hyperplane placement method. Policy 20 has a small section of overlap with the greedy hyperplane placement method, but looks to be better. Policy 21 has non-trivial overlap with the greedy hyperplane placement method, making it very difficult to determine which is better.

\subsection{A Comparison of Policies Vs. Moghrabi’s (2003) Results}

Moghrabi (2003) developed a set of policies which employed multiple greedy hyperplane placement methods during the tree creation process. These methods, along with C4.5 (Quinlan, 1993) were tested against many of the datasets that the policies presented in this thesis were tested against. Those datasets that were tested in both Moghrabi's thesis and this thesis are highlighted in bold in Table 8. Moghrabi's results, 
and more importantly, $\mathrm{C} 4.5$ 's results offer a good benchmark against which to compare the results achieved by the policies presented in this thesis.

Figure 49 and Figure 50 plot various combinations of policies against:

- Moghrabi's Best: Policy \#9 in Moghrabi's (2003) thesis. This policy chose the best tree created by the 8 other policies presented in his thesis. (Discussed in more detail in section 3.2)

- C4.5: a commercial implementation of C4.5 (Quinlan, 1993).

- Greedy: Chinneck's (2009) greedy hyperplane placement method.

There could be some argument about whether or not comparing the results of C4.5 against a combination of policies is a valid comparison. The use of "boosting" and "Or-ing" methods, which is a common practice in commercially available classifiers, results in multiple tree creation from which the best candidate is selected. Thus a comparison against policies that select from numerous candidate trees is not unreasonable.

Figure 49 shows that a combination of three or even two policies can produce results that are clearly better than those achieved by C4.5 or Moghrabi's best policy. The two policy combinations: 4 , and 20 and 3, 4, and 20 were chosen because they accounted for the greatest number of high scores across the 35 datasets tested. 


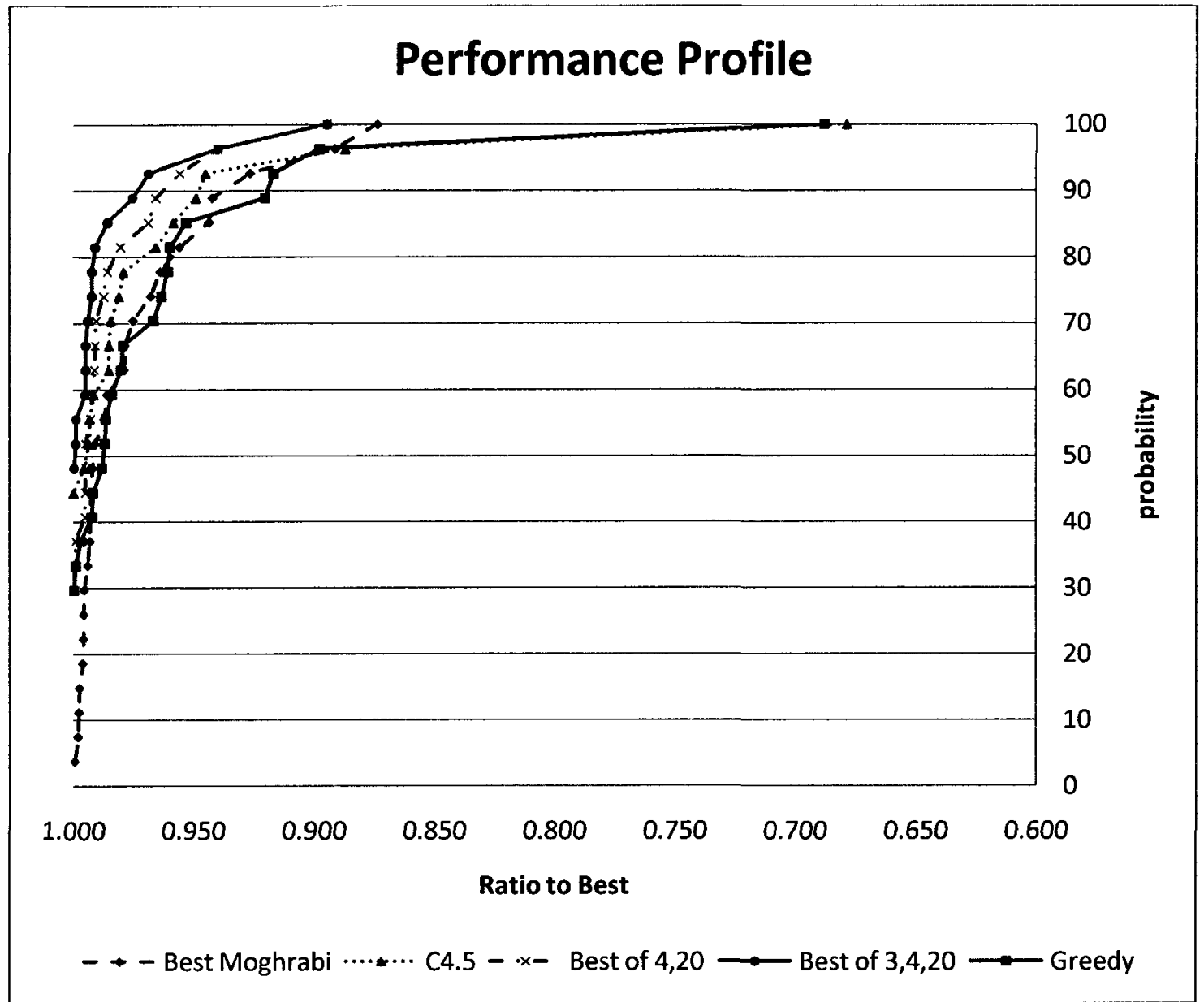

Figure 49: A performance profile comparing the results from Moghrabi's (2003) thesis against various combinations of non-greedy policies.

As can be seen in Figure 50, the best performing non-greedy policies have clearly outperformed the other tree creation methods. 


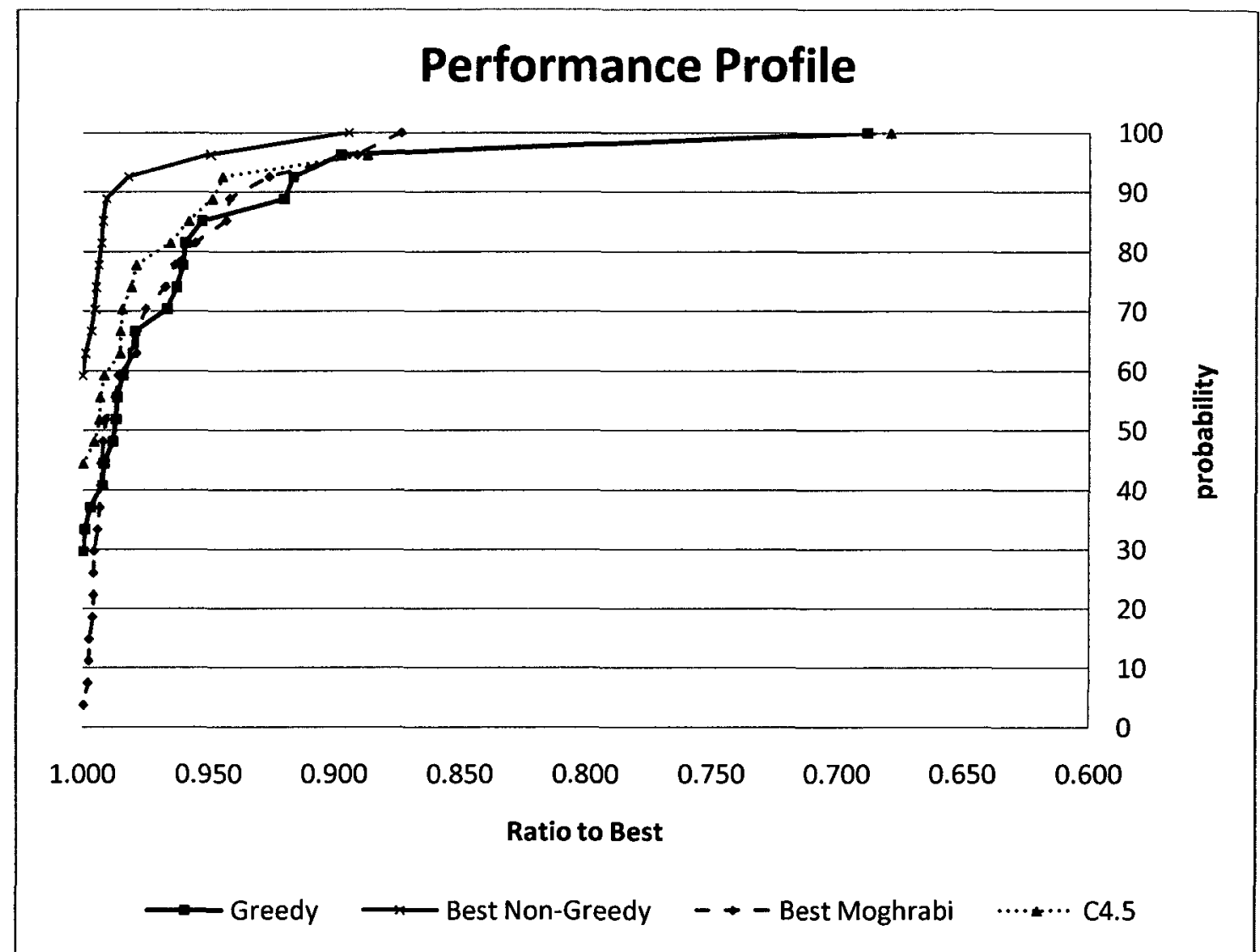

Figure 50: A performance profile comparing the state of the art tree creation methods against the best performing non-greedy policy

\subsection{A Comparison of Policies Vs. Commercial Classifiers}

This comparison plots the performance of several commercial classifiers against the policies presented in this thesis. All commercial classifiers were tested using their default settings with the following exception:

- CART 6.0 was set to maximize for accuracy over compact tree size and required a minimum of 5 data points be present in a node in order to branch.

- GATree was set to allow for the maximum number of generations: 100 . 


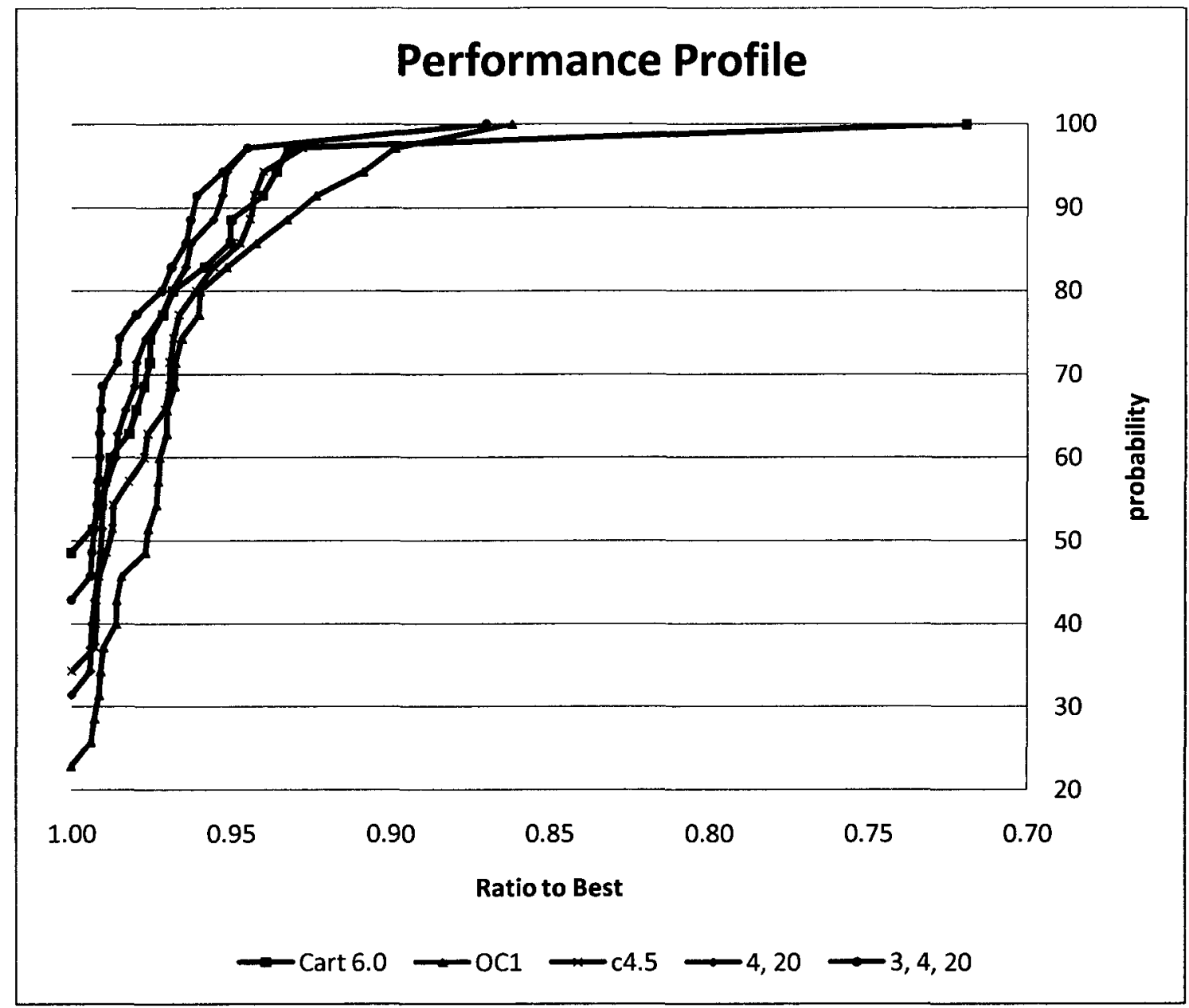

Figure 51: The best of all Policies against freely available commercial classifiers.

Note that in Figure 51, GATree is omitted because its poor performance results in a performance profile curve that is so much worse than the others that the chart becomes difficult to read. Figure 51 shows that a combination of Policies 3, 4 and 20 outperforms all the commercial classifiers, while the combination of Policies 4 and 20 outperforms $\mathrm{OC} 1$ and GATree, and is competitive with Cart 6.0 and C4.5. 


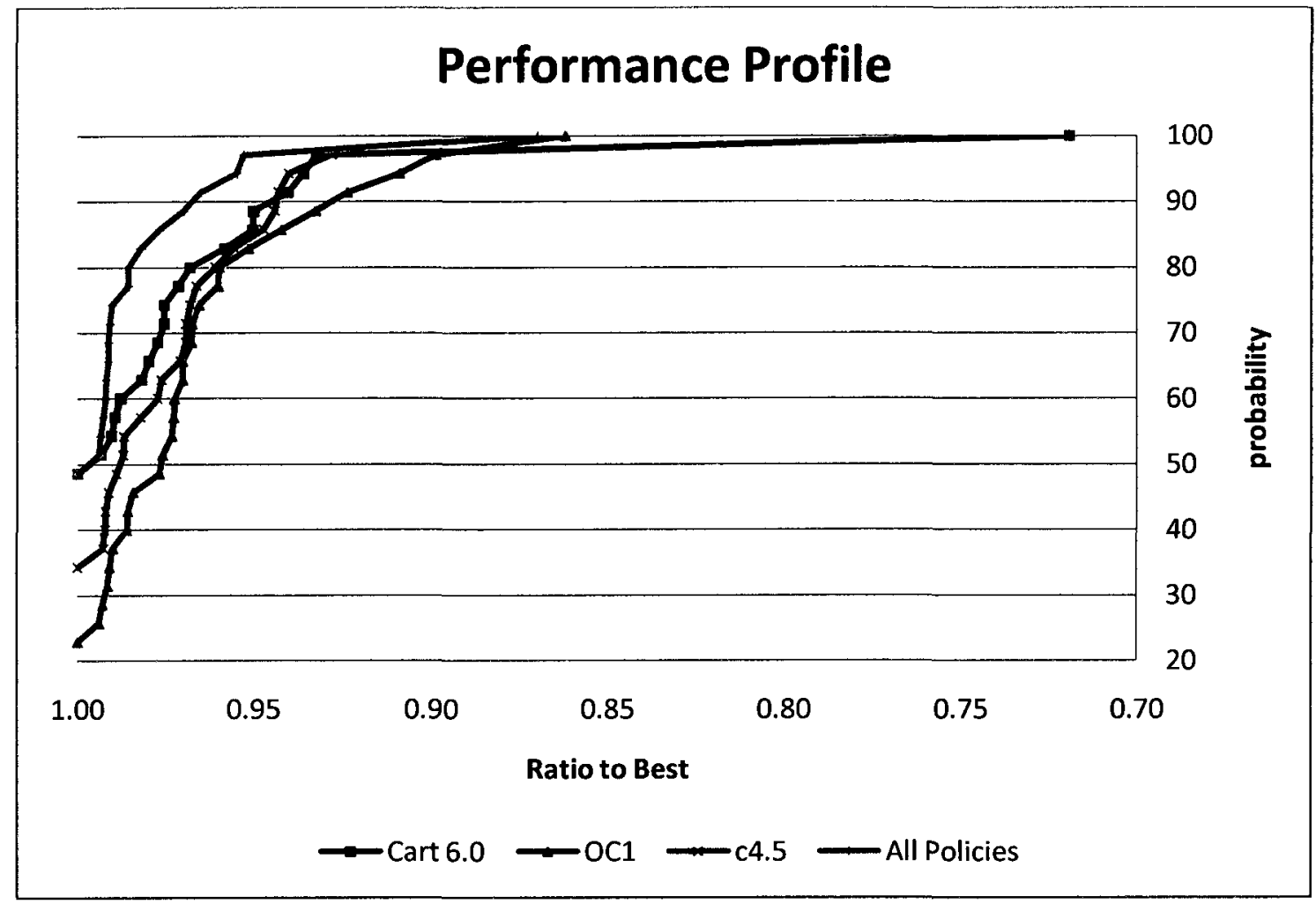

Figure 52: The best of all Policies against freely available commercial classifiers.

As with Figure 51, Figure 52 omits the performance profile for GATree in order to better illustrate the differences between the more competitive classifiers. Figure 52 shows clearly that the best of all Policies outperforms all the commercial classifiers.

\subsection{Tree Creation Times}

When dealing with tree creation algorithms, time is not a critical factor. Once a tree is trained, there is no easy way to make adjustments. If a trained tree is deemed inadequate, then a new tree needs to be created. A binary classification tree that performs a few percentage points better is well worth a significant wait. The tree creation times for the various policies implemented in this thesis are shown in Table 10. 
Table 10: Tree and branch creation times, specified in minutes

\begin{tabular}{|l|c|c|c|c|c|c|c|c|c|}
\cline { 2 - 10 } \multicolumn{1}{c|}{} & \multicolumn{9}{|c|}{ Policies } \\
\hline & Greedy & 3 & 4 & 10 & 13 & 16 & 18 & 20 & 21 \\
\hline $\begin{array}{l}\text { Avg Tree } \\
\text { Creation Time }\end{array}$ & 0.42 & 1.50 & 1.73 & 19.41 & 6.94 & 9.40 & 19.82 & 8.16 & 8.49 \\
\hline $\begin{array}{l}\text { Avg } \\
\begin{array}{l}\text { Hyperplane } \\
\text { Creation Time }\end{array}\end{array}$ & 0.23 & 0.77 & 0.42 & 1.65 & 0.83 & 1.10 & 1.20 & 1.07 & 1.03 \\
\hline
\end{tabular}

Given that Moghrabi's experiments were performed 5 years ago, using less sophisticated hardware, a comparison of the tree creation times achieved in this thesis with his results would be of very limited value. Moghrabi (2003) states that tree creation time took up to 30 hours for one policy to create a binary classification tree. The tree creation times for the policies in this thesis are significantly shorter, but how much of that is due to faster computers, and perhaps a more up to date LP solver is unknown.

Given that the use of multi-core, 64 bit computers is now prevalent, it can be confidently stated that building trees using as many as 3 or 4 policies in parallel can be done in the same amount of time as it takes to build a single tree with the slowest of those policies. 


\section{Conclusions}

\subsection{Conclusions}

This thesis explores the performance of policies that employ non-greedy hyperplane placement methods. The performance of these policies was compared to:

1) A greedy hyperplane placement method

2) Moghrabi's Policy \#9, which employed multiple greedy hyperplane placement methods, and chose the best performing candidate.

3) The performance of several commercial classifiers:
a. C4.5 (Quinlan 1993)
b. Cart 6.0 (Salford Systems, 2009)
c. OC1 (Murthy, 1997)
d. GATree (Athanassios and Dimitrios, 2001)

In addition, an attempt was made to create a set of non-greedy policies that, at any given node, would select a hyperplane placement method from a group of greedy methods such that a better overall tree would be created.

The policies employing non-greedy hyperplane placement methods were tested against other state of the art methods according to the experimental process outlined in Section 1. The following observations arise from the results, which were tabulated in Section 1:

- No single method was found that correctly predicts what greedy hyperplane placement method to use for a given node in every case. 
- Several policies that utilize non-greedy hyperplane placement methods outperform the greedy hyperplane placement method. Policies 3,4 , and 20 stand out as being clearly better.

- Policies making use of Edge Following hyperplane placement methods tended to outperform those that made use of pure cuts or mixed zone cuts. The 3 best performing non-greedy hyperplane placement methods: 3,4 , and 20 all make use of Edge Finding hyperplane placement methods.

- Parallel tree development drastically improves performance of non-greedy policy based tree creation to the point where 2 or 3 such policy based trees developed in parallel outperform the commercial classifiers tested.

- Employing greedy methods when creating leaf nodes results in better overall trees.

\subsection{Contributions}

The main contributions of this thesis are:

- The development of the following new non-greedy hyperplane placement techniques:

- Mixed Zone Cut

- Edge Following Class Isolation Pure Cut

- Edge Following Class Isolation Greedy Cut

- Edge Following Mixed Isolation Pure Cut

- Edge Following Mixed Isolation Greedy Cut

- The development of policies (fixed, partially dynamic and fully dynamic) that apply different hyperplane placement techniques at various nodes during tree construction 
- Development of a hybrid policy that outperforms the state of the art, including several commercial solvers, (i.e. the combination of policies 3,4,20) using the new Edge Following hyperplane placement methods.

\subsection{Future Research}

The work in this thesis represents a first step towards building classification decision trees using non-greedy hyperplane placement techniques, and there are opportunities for significant enhancements. Some possible improvements might be:

1) To employ the "OR" technique used by SMILES (Ferri-Ramírez et al., 2002). A successful implementation of this technique with two policies would guarantee a tree that performs at least as well on any given dataset as the best of the two individual policies.

2) Another option for improvement would be to make use of work by Duckham et al. (2008), which describes and implements an algorithm that traces out a shape based upon a set of points placed upon a plane. Outliers are identified and left out of the calculation of the shape to be drawn. If more than one distinct grouping of points is present, more than one enclosed shape is drawn. This method might select better subsets of data points than the KNN method used by the non-greedy hyperplane placement methods.

3) As discussed in Section 2.1.4.3, pruning tends to produce better trees than stopping criterion. Unfortunately these methods tend to rely on greedy metrics when determining which hyperplanes to keep. A pruning method that accommodates non-greedy hyperplane placement methods is needed. 


\section{Appendix A - Artificial Datasets}

A group of artificial datasets were designed for development purposes. The various sets were designed to take on specific shapes that would pose difficulties to various hyperplane creation methods. These datasets were created by first generating a random set of 500 2-dimensional points. Each dimension's value has a range of -1 to +1 . The various datasets were created by varying the class assigned to these points. Table 11 gives the details for each of these datasets, and Figure 53 to Figure 58 show diagrams representing these datasets.

Table 11: Synthetic Datasets

\begin{tabular}{|l|c|c|c|c|}
\hline \multicolumn{1}{|c|}{ Data Set } & $\begin{array}{c}\text { Total } \\
\text { Points }\end{array}$ & $\begin{array}{c}\text { Number } \\
\text { of Class 0 } \\
\text { pts }\end{array}$ & $\begin{array}{c}\text { Number } \\
\text { of Class 1 } \\
\text { pts }\end{array}$ & predictors \\
\hline BullsEye & 500 & 100 & 400 & 2 \\
\hline BullsEyem & 500 & 111 & 389 & 2 \\
\hline Diagonalsplit & 500 & 243 & 257 & 2 \\
\hline Diagonalsplitm & 500 & 240 & 260 & 2 \\
\hline Halfhourglass & 500 & 437 & 63 & 2 \\
\hline Halfhourglassm & 500 & 455 & 45 & 2 \\
\hline Hourglass & 500 & 247 & 253 & 2 \\
\hline Hourglassm & 500 & 309 & 191 & 2 \\
\hline Lefthalfplane & 500 & 250 & 250 & 2 \\
\hline Lefthalfplanem & 500 & 231 & 269 & 2 \\
\hline Q14 & 500 & 265 & 235 & 2 \\
\hline Q14m & 500 & 241 & 259 & 2 \\
\hline OddBullsEye & 500 & 192 & 308 & 2 \\
\hline
\end{tabular}



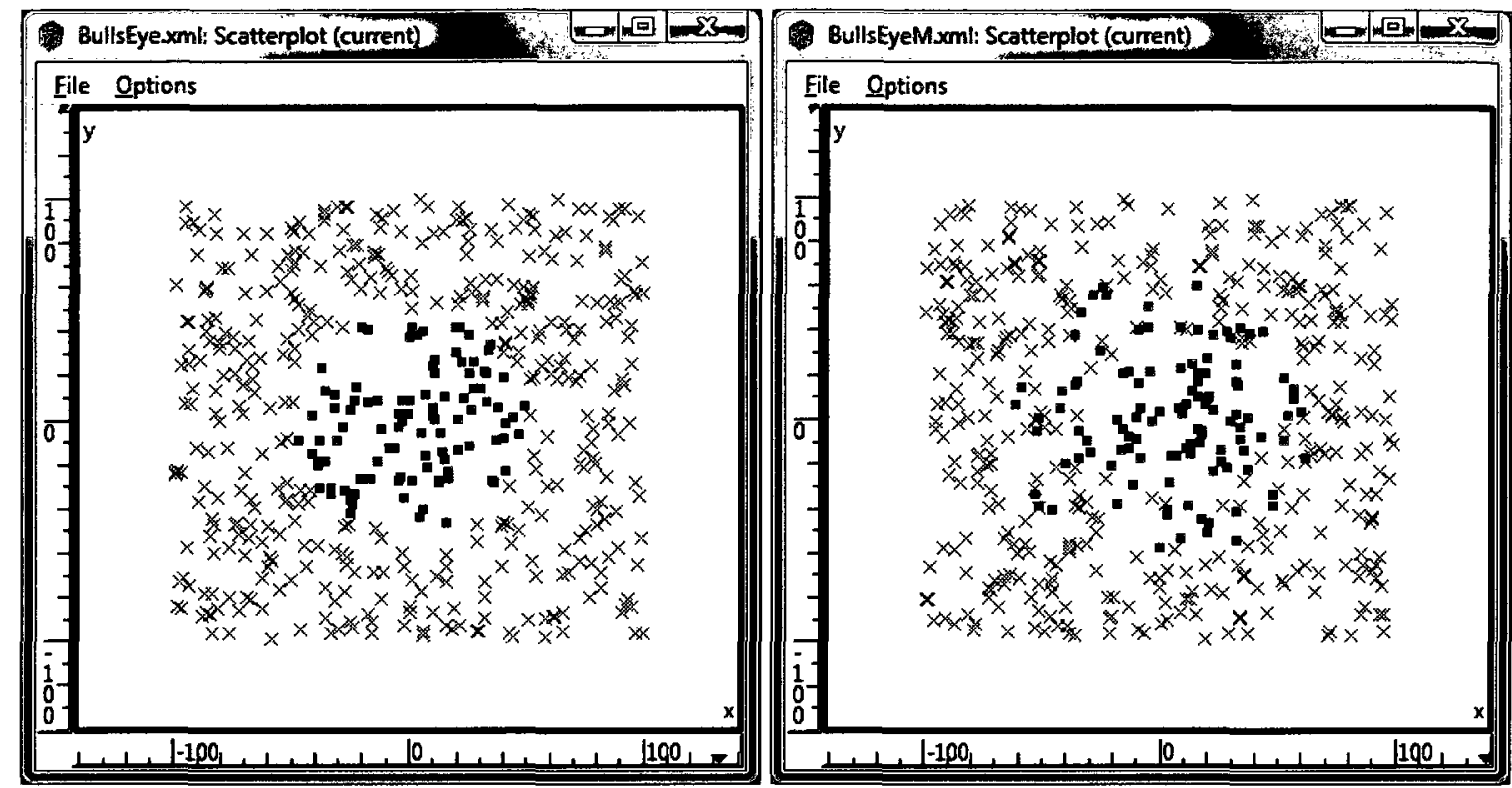

Figure 53: Bullseye and Bullseyem datasets
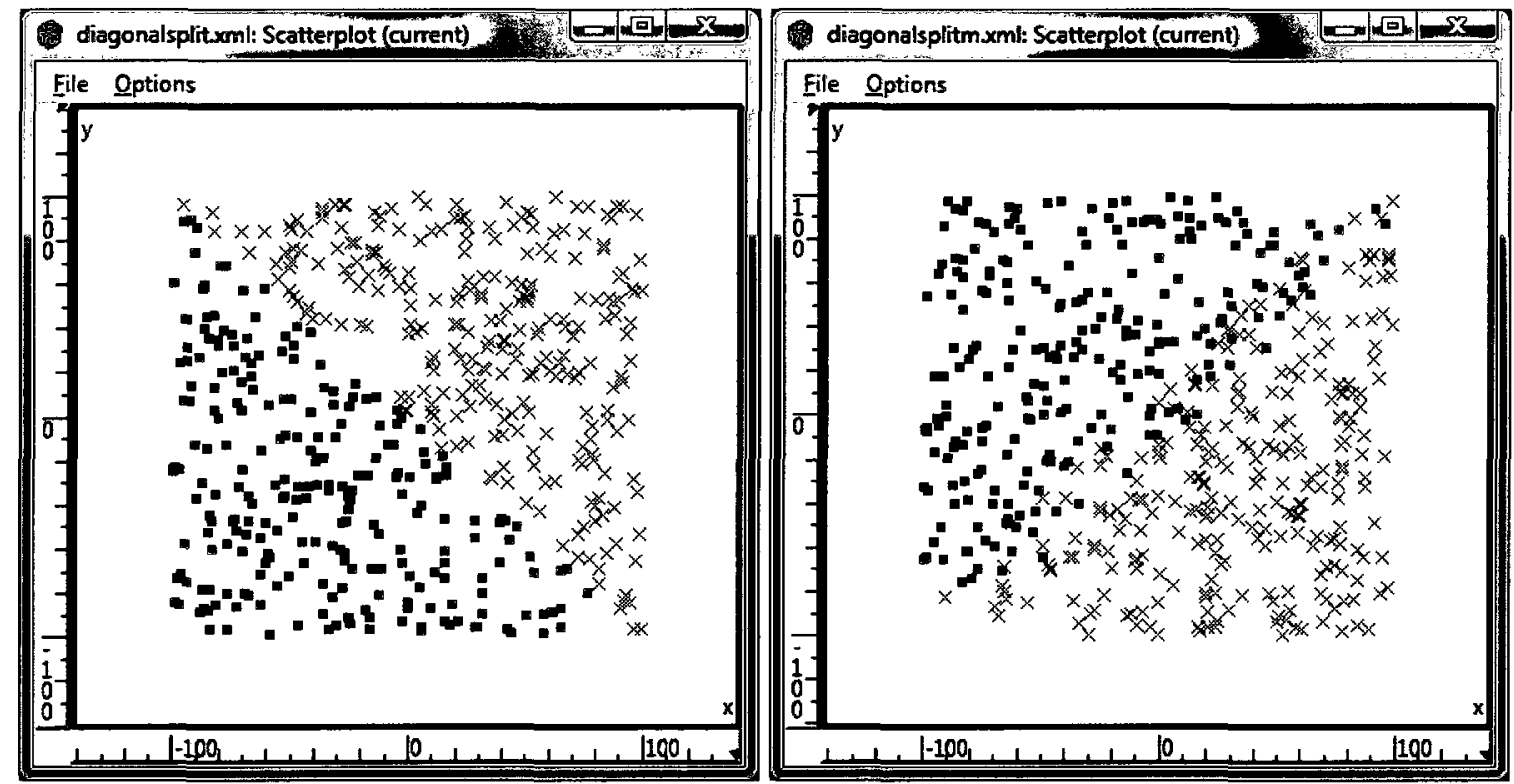

Figure 54: Diagonalsplit and Diagonalsplitm datasets 

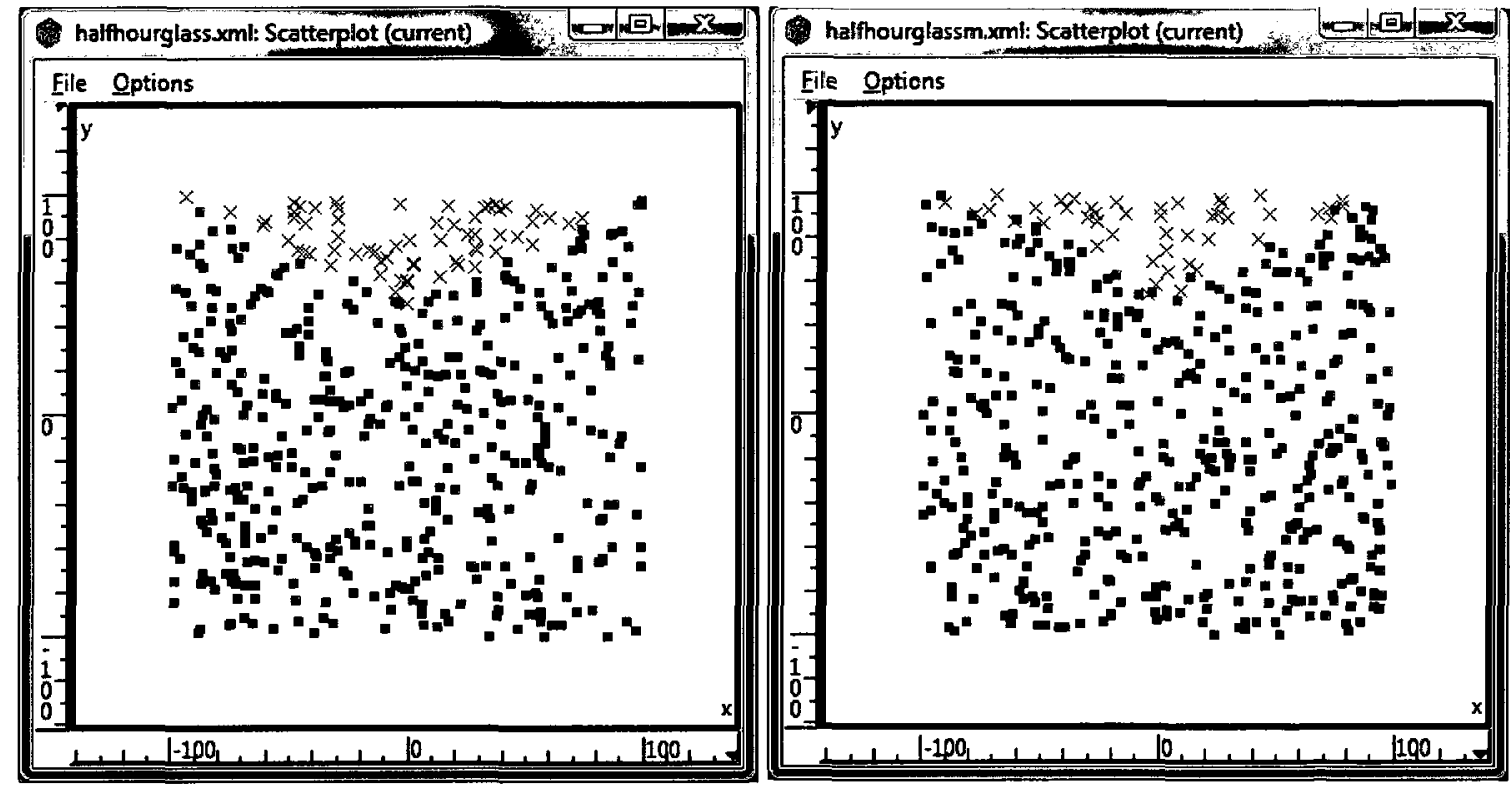

Figure 55: Halfhourglass and Halfhourglassm datasets
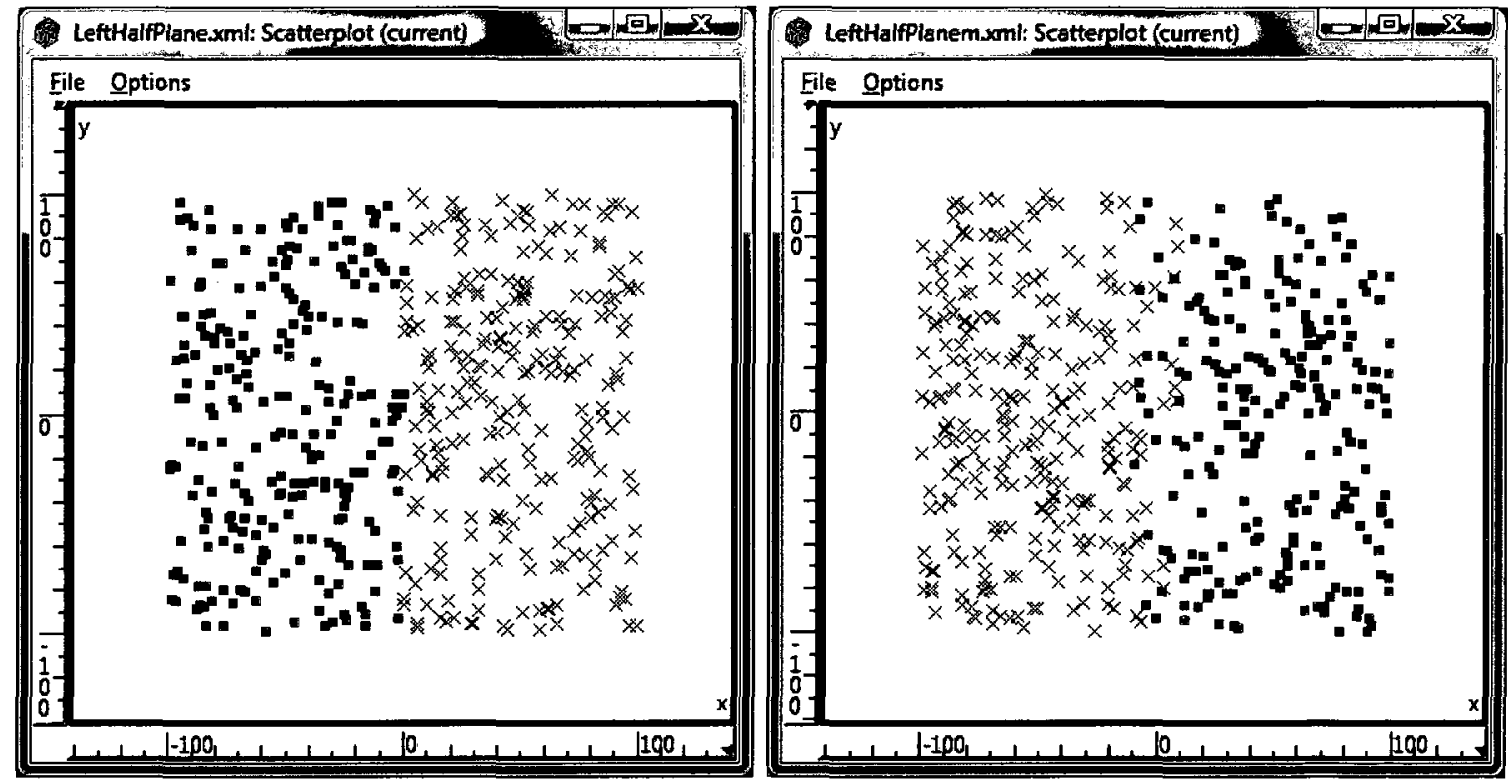

Figure 56: Lefthalfplane and Lefthalfplanem datasets 

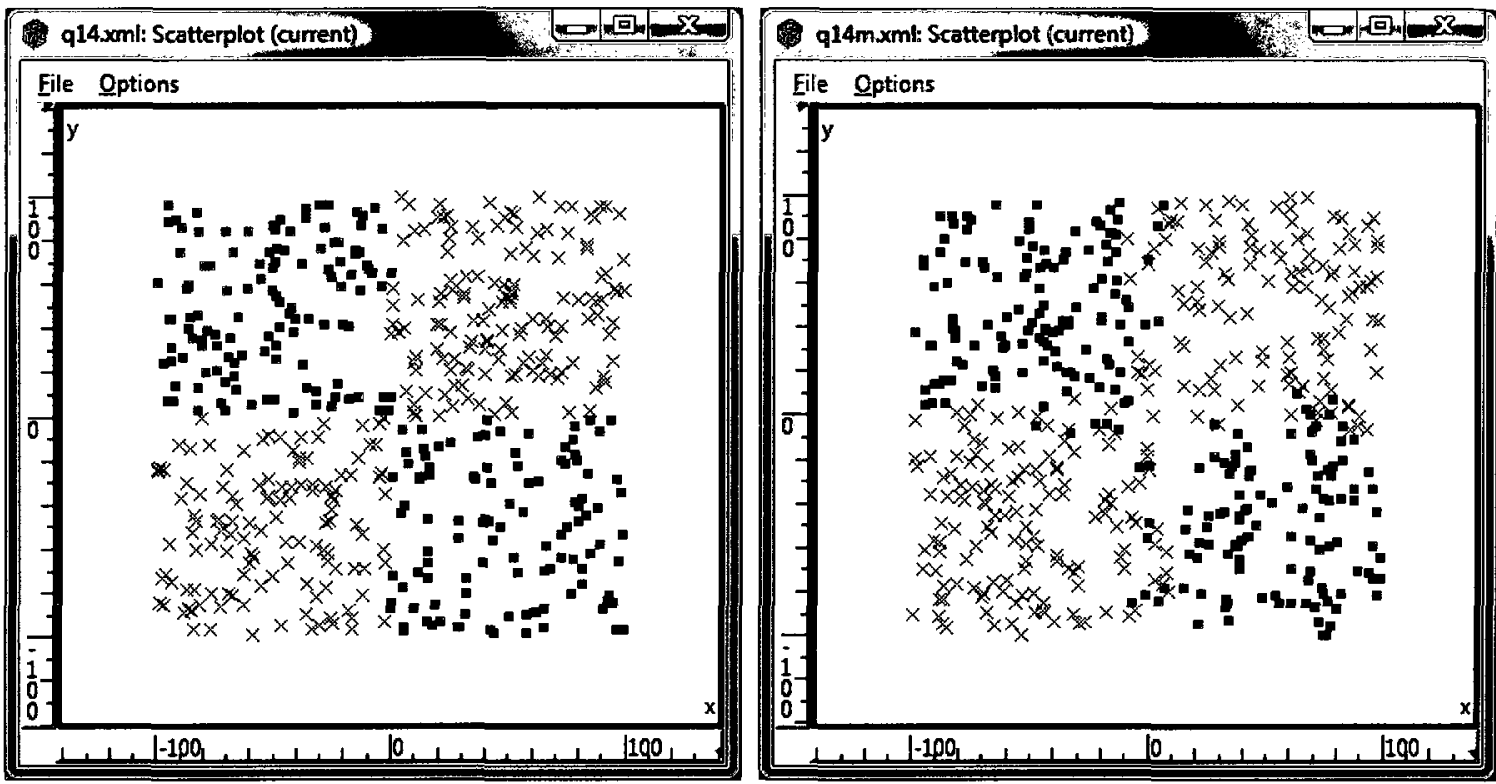

Figure 57: $Q 14$ and $Q 14 m$ datasets

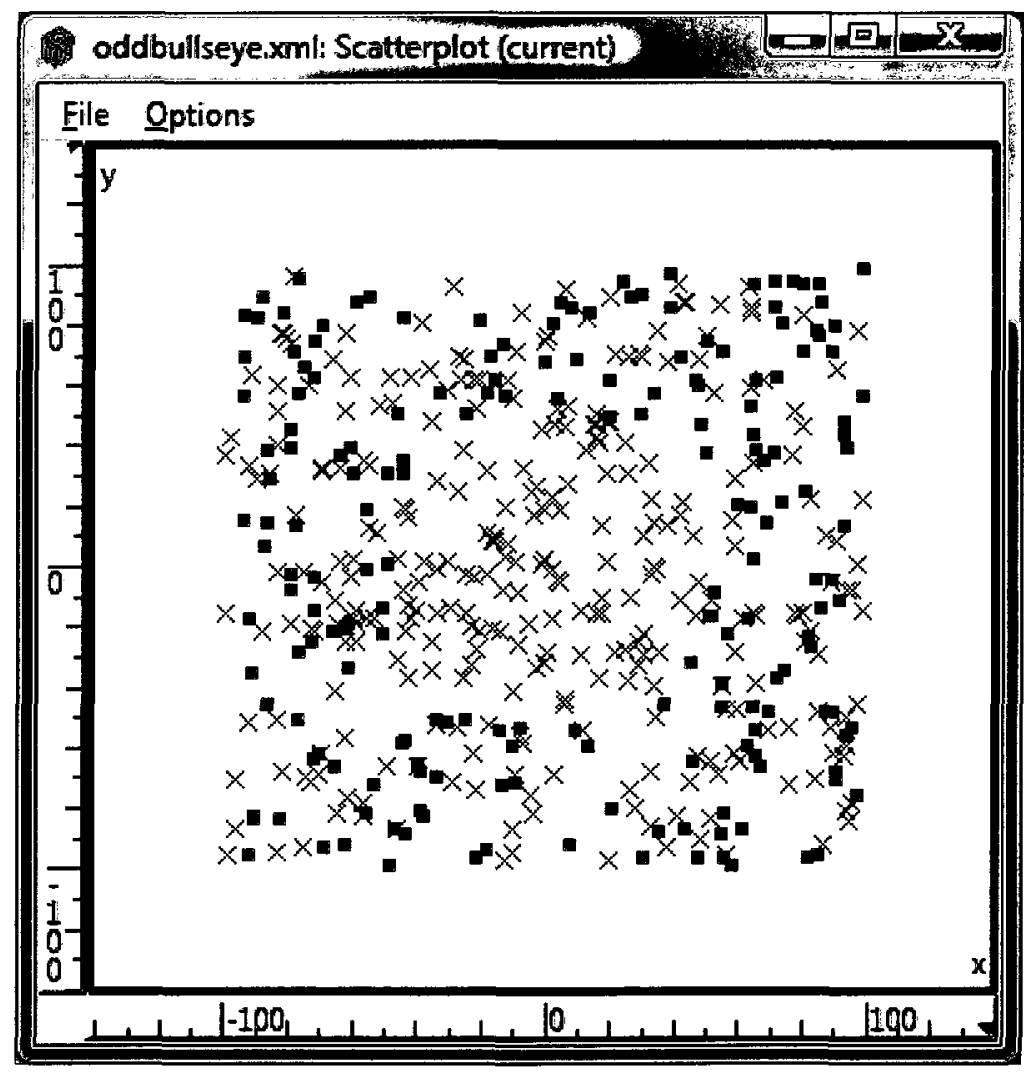

Figure 58: OddBullsEye dataset. 


\section{References}

Athanassios, Papagelis; Dimitrios, Kalles. "Breeding Decision Trees Using Evolutionary Techniques." Proceedings of the Eighteenth International Conference on Machine Learning. (2001), pp. 393-400

Bennet, Kristin. "Tree Optimization: A non-Greedy Decision Tree Algorithm." Computing Science and Statistics. (1994) pp. 156-160

Bramer, Max. Principles of Data Mining. London: Springer, 2007

Breiman, Leo; Friedman, Jerome; Olshen, Richard; Stone, Charles. Classification and Regression Trees. London: Chapman \& Hall/CRC, 1984.

Chinneck, John. "An Effective Polynomial-Time Heuristic for the Minimum-Cardinality IIS-Set-Covering Problem." Annals of Mathematics and Artificial Intelligence. VOL. 17, NO. 1, (1996) pp. 127-144

Chinneck, John. Improved Linear Classification via LP Infeasibility Analysis. Technical Report. Ottawa: Carleton University, Department of Systems and Computer Engineering, 1998

Chinneck, John. "Fast Heuristics for the Maximum Feasible Subsystem Problem." Informs Journal On Computing. VOL. 13, NO. 3 (2001) pp. 210-223

Chinneck, John. "Tailoring Classifier Hyperplanes to General Metrics", in Chinneck, John; Kristjansson, Bjarni; Saltzman, Matthew (eds.), Operations Research and CyberInfrastructure. New York: Springer Science+Business Media, 2008

Chinneck, John. Feasibility and Infeasibility in Optimization: Algorithms and Computational Methods. International Series in Operations Research and Management Sciences. VOL. 118 (2009) pp. 365-387

Gini, Corrado. "Measurement of Inequality of Incomes" The Economic Journal. VOL. 31 NO. 121 (1921) pp. 124-126

Cover, T.M., Hart, Peter. "Nearest Neighbour Pattern Classification." IEEE Trans. Inform. Theory. VOL. IT-13 NO. 1 (1967) pp. 21-27

DMS. Decision Trees. http://dms.irb.hr/tutorial/tut_dtrees.php, 2001

Dolan, Elizabeth; More, Jorge. "Benchmarking Optimization Software with Performance Profiles." Mathematical Programming - Series A. VOL 9, (2002) pp. 201-213 
Duckham, Matt; Kulik, Lars; Worboys, Mark. "Efficient generation of simple polygons for characterizing the shape of a set of points in the plane." Pattern Recognition. VOL 41 NO 10, (2008) pp. 3224-3236

Duda, Richard; Hart, Peter. Pattern Classification and Scene Analysis. New York: Wiley, 1973

Esposito, Floriana; Malerba, Donato; Semera, Giovanni. "A Comparative Analysis of Methods for Pruning Decision Trees." IEEE Transactions On Pattern Analysis And Machine Intelligence. VOL. 19, NO. 5 (1997) pp.1-16.

Ferri-Ramírez, Cesar; Hernández-Orallo, Jose; Ramírez-Quintana, Jose. SMILES v.2.3. A Multi-purpose Learning System. València: Univ. Politècnica de València, 2002

Forrest, John. CLP. https://projects.coin-or.org/Clp/wiki, 2009

Geisser, Seymour. "The Predictive Sample Reuse Method with Applications." Journal of the American Statistical Association. VOL. 70, NO. 350 (1975) pp. 320-328

Gowan, M. Gini coefficient. http://en.wikipedia.org/wiki/Gini_index, 2008

Kim, Hyunjoong; Loh, Wei-yin. "Classification Trees With Unbiased Multiway Splits." Journal of the American Statistical Association. VOL. 96 (2001) pp. 589-604.

Lazaridès, Ariane; Normandin, Yves; Kuhn, Roland. "Improving Decision Trees For Acoustic Modeling." Fourth International Conference on Spoken Language. VOL. 2 (1996) pp. 1053-1056

Ling, Charles; Sheng, Shengli. "Hybrid Cost-Sensitive Decision Tree." Lecture Notes in Computer Science. VOL 3721, (2005) pp. 274-284

Loh, Wei-Yin; Shih, Yu-Shan. "Split Selection Methods for Classification Trees." Statistica Sinica.VOL. 7 (1997) pp. 815-840

Metz, Charles. "Basic Principles of ROC Analysis." Seminars in Nuclear Medicine. VOL 7 NO 4 (1978) pp. 283-298

Microsoft. Visual Studio 2008. http://msdn.microsoft.com/en-us/vstudio/default.aspx, 2008

Moghrabi, Ahmad. Adaptive Construction of Classification Trees. Masters Thesis. Ottawa: Carleton University, 2003

Murthy, Sreerama. On Growing Better Decision Trees from Data. PhD Thesis, Baltimore: John Hopkins University, 1997 
Quinlan, Ross. "Induction of Decision Trees." Machine Learning. VOL. 1 NO. 1 (1986) pp.81-106

Quinlan, Ross. C4.5: Programs for Machine Learning. Palo Alto, CA: Morgan Kaufmann, 1993

Quinlan, Ross. Data Mining Tools See5 and C5.0. http://www.rulequest.com/see5info.html, 2002

Rokach, Lior; Maimon, Oded. "Top-Down Induction of Decision Trees Classifiers - A Survey." IEEE Transactions On, Man, and Cybernetics: Part C. VOL. 1, NO. 11 (2002) pp. $476-487$

Safavian, Rasoule; Landgrebe, David. "A Survey of Decision Tree Classifier Methodology." IEEE Transactions on Systems, Man, and Cybernetics. VOL. 21, NO 3 (1991) pp. 660-674

Salford Systems. CART. http://www.salfordsystems.com/cart.php, 2009

Schapire, Robert. "A Brief Introduction to Boosting." Proceedings of the Sixteenth International Joint Conference on Artificial Intelligence. (1989) pp. 1401-1406

Stinson, Douglas. Cryptography Theory and Practice. New York: Chapman \& Hall, 2002

University of California at Irvine. Machine Learning Repository. http://archive.ics.uci.edu/ml/, 2009 